\title{
The Evolution of Wahlenbergia (Campanulaceae) in Australasia
}

By

Jessica Mary Prebble

A thesis submitted to the Victoria University of Wellington in fulfilment of the requirement for the degree of Masters in Ecology and Biodiversity

Te Whare Wananga o te Upoko o te Ika a Maui April 2010 



\section{Abstract}

Wahlenbergia is a large genus of flowering plants within the family Campanulaceae. In this thesis the first molecular phylogeny of Wahlenbergia was reconstructed from approximately $20 \%$ of the genus, based on the nuclear ribosomal ITS (nrITS) DNA marker and the chloroplast trnL-F DNA marker, with samples from South Africa, Europe, Australia and New Zealand. Additionally a large phylogeny with increased within-species sampling focusing on addressing taxonomic questions among the 45 Australasian species of Wahlenbergia was also reconstructed based on nrITS and $\operatorname{trn} L-F$, plus an additional chloroplast DNA marker, $\operatorname{trnK}$. Relationships and species limits of the New Zealand species of Wahlenbergia were further analysed using amplified fragment length polymorphisms (AFLPs).

Wahlenbergia was found to be polyphyletic, though most of the species form a clade. Tree topologies and molecular dating analysis showed that the genus originated in South Africa about 16.2 million years ago (mya), then dispersed to Australasia before radiating there about 3.7 mya, thus refuting the hypothesis of Gondwanan vicariance for the Australasian species. Two dispersals from Australia to New Zealand are hypothesised, one leading to a radiation of species with the rhizomatous growth from about 1.0 mya and the other leading to a radiation of species with the radicate growth form 0.49 mya, although the radicate species might not form a clade. Low levels of genetic variation among individuals from Australia and New Zealand was revealed with all markers, and the phylogenies were poorly resolved as a result. The low genetic diversity is probably due to rapid and recent evolution during a period of geological and climatic change, coupled with incomplete lineage sorting and hybridisation. Phylogenies reconstructed using AFLPs were also poorly resolved, although AFLPs were found to be useful for species delimitation, as has been shown in studies of other plant groups.

Despite the poor resolution, several morphological species and subspecies were recovered as monophyletic with DNA sequence data, notably the morphologically distinctive New Zealand $W$. cartilaginea, W. matthewsii and $W$. congesta subsp. 
congesta. Further research into species boundaries within the W. albomarginata/W. pygmaea complex is needed. Members of the New Zealand lowland radicate $W$. gracilis complex may all belong to the same morphologically variable species, although further research is needed to justify such a taxonomic change. The other New Zealand radicate species, $W$. vernicosa, is probably a separately evolving lineage, and is not conspecific with the Australian W. littoricola. 


\section{Acknowledgements}

I would like to express my sincere thanks to the many people who have helped me prepare this thesis. I would especially like to thank my supervisors, Heidi Meudt and Phil Garnock-Jones, for your generous support, guidance and endless re-reading of drafts. Chris Cupido kindly shared his DNA sequences of South African Wahlenbergia, as well as his knowledge of the genus.

Thank you to Peter de Lange, Ewen Cameron, Mike Bayly, David Lyttle, Graeme Atkins, Hugh Wilson, Jeremy Bruhl, Benjamin Magaña-Rodríguez, Hamish Carson, Tony Silbery, Phil Garnock-Jones and Heidi Meudt for helping with specimen collection, and to all my field assistants: Gesine Pufal, Teresa Herleth, Mark Prebble, Lesley Bagnall, Fiona Hodge and Meg Prebble. Graham Petterson generously shared Judith Petterson's notes and photographs of Wahlenbergia, as well as some plants. Thanks also to helpful DoC staff all around the country. Barbara Briggs and others at the Sydney Botanical Gardens thoughtfully organised a NSW collection permit for me, and Mike Bayly and Alison Kellow provided accommodation in Melbourne.

I am thankful to the Institute of Natural Resources and the Allan Wilson Centre at Massey University for use of the Ancient DNA Facility, and especially Dr Steve Trewick for advice. Barbara Holland gave invaluable help running python scripts for the AFLP chapter. A big thanks to my proof-readers and formatters, Travis LealandMapelsden, Lesley Bagnall and Aaron Packard.

I am grateful to the Auckland Botanical Society for granting me their Lucy Cranwell field grant, to Te Papa for their Te Papa MSc scholarship in molecular systematics and to Victoria University for the VUW post-grad scholarship. Everybody at the Te Papa herbarium has also been especially supportive.

Finally, thank you to my lovely family and friends. 


\section{Table of Contents}

ABSTRACT III

ACKNOWLEDGEMENTS V V

$\begin{array}{lll}\text { CONTENTS } & \text { VI }\end{array}$

CHAPTER ONE: GENERAL INTRODUCTION 1

The New Zealand flora: origins, biogeography and evolution 1

Molecular phylogenetics $\quad 3$

Choosing DNA markers for molecular phylogenetic study 5

Organellar DNA (chloroplast and mitochondrial)

$\begin{array}{ll}\text { Nuclear ribosomal DNA } & 6\end{array}$

$\begin{array}{ll}\text { Low-copy nuclear markers } & 7\end{array}$

AFLPs $\quad 8$

$\begin{array}{lr}\text { Molecular clocks } & 9\end{array}$

$\begin{array}{lr}\text { Introduction to study group } & \mathbf{1 0}\end{array}$

Brief taxonomic history of Wahlenbergia in New Zealand 13

$\begin{array}{lr}\text { New Zealand radicate species } & 15\end{array}$

$\begin{array}{ll}\text { New Zealand rhizomatous species } & 17\end{array}$

$\begin{array}{ll}\text { A note on species concepts } & 17\end{array}$

$\begin{array}{lr}\text { Study aims } & 18\end{array}$

CHAPTER 2: BIOGEOGRAPHY AND A FIRST PHYLOGENY OF WAHLENBERGIA (CAMPANULACEAE) 19

$\begin{array}{lr}\text { Abstract } & 19\end{array}$

$\begin{array}{lr}\text { Introduction } & 19\end{array}$

Materials and Methods $\quad \mathbf{2 2}$

Genetic markers $\quad 22$

Study group $\quad 23$

DNA extraction, amplification and sequencing $\quad 24$

Dataset alignment and analysis $\quad 25$

$\begin{array}{ll}\text { Phylogenetic and dating analysis } & 25\end{array}$

$\begin{array}{ll}\text { Biogeography and character mapping } & 27\end{array}$

Results $\quad 28$

Nuclear (ITS) phylogeny $\quad 28$

$\begin{array}{lr}\text { Chloroplast }(t r n L-F) \text { phylogeny } & 28\end{array}$

Analysis of concatenated dataset $\quad 30$

Combined dataset $\quad 32$

Molecular dating analysis $\quad 32$ 
Biogeography and character mapping $\quad 34$

$\begin{array}{ll}\text { Discussion } & 35\end{array}$

Summary and Conclusions $\quad 39$

CHAPTER THREE: PHYLOGENETIC ANALYSIS OF THREE DNA SEQUENCING MARKERS TO EXPLORE RELATIONSHIPS WITHIN AUSTRALASIAN WAHLENBERGIA

$\begin{array}{ll}\text { Abstract } & 41\end{array}$

Introduction $\quad \mathbf{4 2}$

Research aims $\quad 43$

Taxonomic introduction $\quad 44$

New Zealand radicate species $\quad 44$

New Zealand rhizomatous species $\quad 45$

Australian species $\quad 48$

Methods $\quad 49$

Taxon sampling $\quad 49$

DNA isolation, amplification and sequencing $\quad 49$

Sequence alignment and phylogenetic analysis $\quad 51$

Biogeographic and character analysis $\quad 53$

Results $\quad 53$

DNA extractions and primer selection $\quad 53$

aDNA extractions $\quad 54$

ITS analysis $\quad 59$

$\begin{array}{ll}\text { Chloroplast markers analysis } & 62\end{array}$

Analysis of concatenated dataset $\quad 66$

Combined ITS, trnK and trnL-F analysis $\quad 67$

Discussion $\quad 69$

Evolution of Australasian Wahlenbergia $\quad 69$

Testing the New Zealand Wahlenbergia taxonomy 71

$\begin{array}{ll}\text { New Zealand radicate species } & 71\end{array}$

The position of W. littoricola subsp. vernicosa $\quad 73$

New Zealand rhizomatous species $\quad 74$

Notes on the Australian Wahlenbergia taxonomy 76

$\begin{array}{ll}\text { Australian radicate species } & 76\end{array}$

$\begin{array}{ll}\text { Australian rhizomatous species } & 78\end{array}$

$\begin{array}{ll}\text { Newly described species } & 78\end{array}$

$\begin{array}{lr}\text { Summary and conclusions } & 79\end{array}$

CHAPTER FOUR: USING AFLPS TO RESOLVE PHYLOGENETIC

RELATIONSHIPS IN A PLANT SPECIES COMPLEX WHEN NUCLEAR AND CHLOROPLAST GENES FAIL TO REVEAL SUFFICIENT VARIABILITY81

$\begin{array}{lr}\text { Abstract } & 81\end{array}$

$\begin{array}{lr}\text { Introduction } & 81\end{array}$ 
Sampling and DNA extraction $\quad 84$

Generation of AFLP data $\quad 85$

Parameter optimisation and automated scoring of AFLP data 86

$\begin{array}{ll}\text { Phylogenetic analysis } & 88\end{array}$

Principal coordinate analysis (PCoA) of dominant data 89

$\begin{array}{lr}\text { Results } & 89\end{array}$

Selection of optimal AFLP automated scoring parameters $\quad 89$

Phylogeny of New Zealand Wahlenbergia based on AFLP data 94

$\begin{array}{lr}\text { Cluster analysis using principal coordinates analysis } & 101\end{array}$

Discussion $\quad 98$

Phylogenetic patterns and species delimitation in New Zealand Wahlenbergia based $\begin{array}{ll}\text { on AFLP } & 101\end{array}$

$\begin{array}{ll}\text { Radicate species } & 101\end{array}$

$\begin{array}{ll}\text { Rhizomatous species } & 103\end{array}$

$\begin{array}{ll}\text { Trans-Tasman relationships } & 104\end{array}$

$\begin{array}{ll}\text { Summary and conclusions } & 104\end{array}$

$\begin{array}{ll}\text { CHAPTER FIVE: GENERAL DISCUSSION } & 106\end{array}$

$\begin{array}{ll}\text { Large scale phylogeny and biogeography } & 106\end{array}$

$\begin{array}{ll}\text { Medium and small scale: Relationships between species and taxonomy } & 107\end{array}$

Assessment of molecular techniques used in this thesis 113

$\begin{array}{lr}\text { Future directions } & 113\end{array}$

$\begin{array}{lr}\text { Conclusions } & 115\end{array}$

$\begin{array}{ll}\text { REFERENCES } & 116\end{array}$

$\begin{array}{lr}\text { APPENDIX } & 136\end{array}$

Appendix 1 Table showing details of individuals used in each analysis 136

$\begin{array}{ll}\text { Appendix 2.1 ITS neighbour net network } & 144\end{array}$

$\begin{array}{ll}\text { Appendix } 2.2 \text { trn } L-F+\operatorname{trn} K \text { neighbour net network } & 145\end{array}$

Appendix 2.3 Combined ITS+trnL-F+trnK neighbour net network 146 


\section{LIST OF TABLES}

Table 1.1 Taxonomic treatments of New Zealand Wahlenbergia

Table 3.1 Primer sequences and references for 22 primer pairs trialled in this study

Table 3.2 Uncorrected p distances calculated from the ITS and trnL-F+trnK Australasian Wahlenbergia datasets

Table 4.1 Dataset statistics for the 36 AFLP datasets generated in GeneMarker including and excluding 6FAM characters

Table 5.1 Synthesis table regarding species delimitation

\section{LIST OF FIGURES}

Figure 1.1 Growth forms of New Zealand Wahlenbergia

Figure 1.2 Photos of New Zealand Wahlenbergia

Figure 2.1 Bayesian 50\% majority rule consensus phylogeny of Wahlenbergia samples from South Africa, Europe, Australia and New Zealand reconstructed using ITS

Figure 2.2 Bayesian 50\% majority rule consensus phylogeny of Wahlenbergia samples from South Africa, Europe, Australia and New Zealand reconstructed using trnL-F

Figure 2.3 Bayesian maximum clade credibility tree of Wahlenbergia samples from South Africa, Europe, Australia and New Zealand reconstructed using a concatenated ITS $+\operatorname{trn} L-F$ dataset, showing divergence time estimates and with growth forms and locations mapped

Figure 3.1 Photographs of gels showing chloroplast markers trialled

Figure 3.2 Photographs of gels showing nuclear and mitochondrial markers trialled

Figure 3.3 Bayesian 50\% majority rule consensus phylogeny of Wahlenbergia samples from Australasia reconstructed using ITS, with MP and ML bootstrap values added

Figure 3.4 Bayesian 50\% majority rule consensus phylogeny of Wahlenbergia samples from Australasia reconstructed using $\operatorname{trn} L-F$, with MP and ML bootstrap values added

Figure 3.5 Bayesian 50\% majority rule consensus phylogeny of Wahlenbergia samples from Australasia reconstructed using a combined ITS $+\operatorname{trn} L-F+\operatorname{trn} K$ dataset, with MP and ML bootstrap values added 68

Figure 4.1 Consensus network of the 36 AFLP datasets generated in GeneMarker excluding 6FAM characters

Figure 4.2 One of the five most parsimonious trees reconstructed from Australasian Wahlenbergia AFLP data with bootstrap values greater than $50 \%$ added

Figure 4.3 Bayesian 50\% majority rule consensus phylogeny of Australasian Wahlenbergia AFLP data showing posterior probability values 
Figure 4.4 Principle coordinates analysis of Australasian Wahlenbergia AFLP data showing three dimensions

Figure 4.5 Principle coordinates analysis of a reduced Australasian Wahlenbergia AFLP dataset showing the W. albomarginata/W. pygmaea complex in three dimensions

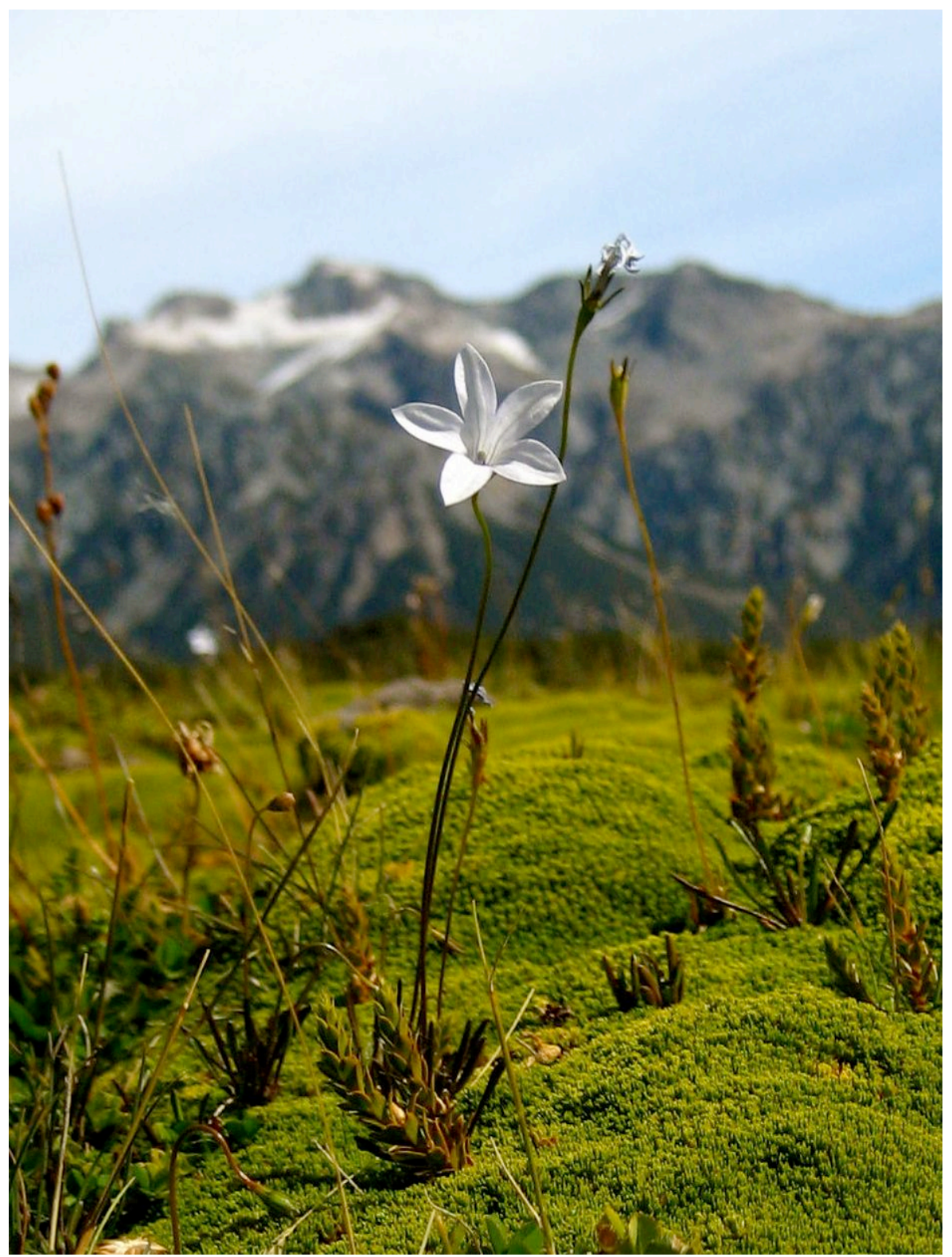

Wahlenbergia albomarginata subsp. albomarginata growing on the Waimakariri riverbed, near Arthur's Pass 


\section{Chapter One: General Introduction}

\section{The New Zealand flora: origins, biogeography and evolution}

The New Zealand flora has its roots on the ancient supercontinent Gondwanaland, yet New Zealand has been increasingly isolated over the last 80 million years (my), and has been separated from its nearest large neighbour, Australia, by $1500 \mathrm{~km}$ for at least 60 my (Kamp, 1986; Wallis and Trewick, 2009; Winkworth et al., 2005). This long isolation has fostered debate regarding the origins of the flora, a debate that became unnecessarily polarised for a time (Wallis and Trewick, 2009) between supporters of vicariance (Wardle, 1963), and long distance dispersal (Pole, 1994). It is generally accepted that a mixture of the two modes has affected the formation of the New Zealand flora as we know it, though Pole (1994) and Macphail (1997) suggested that all of the flora must have arrived in New Zealand by long distance dispersal, as they contend that all of Zealandia (New Zealand plus its large continental shelf) would have been under water during the Oligocene period (the "Oligocene drowning"). There is indeed molecular phylogenetic evidence that a large proportion of the flora has arrived by long distance dispersal after the separation of Zealandia from Gondwanaland (Winkworth et al., 1999), but nevertheless this hypothesis disregards fossil and molecular evidence that suggests some plant lineages e.g. kauri (Agathis) do have a Gondwanan history (Knapp et al., 2007).

Many of the New Zealand plant groups that have a long-distance dispersal event in their history have arrived from Australia (e.g. (Ford et al., 2007; Wagstaff et al., 1999)). This west to east direction of dispersal is consistent with the forces of the west wind drift, which predicts more dispersal events from west to east because of westerly winds and ocean currents (Raven, 1973; Sanmartín et al., 2007; Winkworth et al., 2002b). These New Zealand plant groups are often found to have been 
established by a single dispersal event to New Zealand (implicated if the New Zealand representatives form a monophyletic group) followed by rapid species radiation e.g. (Ford et al., 2007). There is often little DNA sequence divergence between species from Australia and species from New Zealand e.g. Sophora (Hurr et al., 1999), Veronica (Wagstaff et al., 2002) and others (Ford et al., 2007; Sanmartín et al., 2007). Other locations have also been implicated as being the origins of long distance dispersal events to New Zealand, e.g. Fuchsia (Berry et al., 2004), Gaultheria (Bush et al., 2009) and the tribe Coronanthereae (Gesneriaceae) (Woo, 2007) from South America. However, these events are rarer than introductions from Australia and likely involve Antarctica as a stepping stone while it was still partly ice-free and connected to Australia. There are also examples of multiple introductions from Australia e.g. Drosera (Rivadavia et al., 2003) and Plantago (Tay et al., 2010).

There is molecular phylogenetic evidence from multiple studies that the ancestors of many of these plant groups radiated rapidly once they established in New Zealand (Winkworth et al., 1999) e.g. Veronica (Wagstaff and Garnock-Jones, 1998, 2000), Pachycladon (Heenan et al., 2002), Polystichum (Perrie et al., 2003), Pratia (Murray et al., 2004) and Ourisia (Meudt et al., 2009). These radiations are often associated with speciation after geologically recent long distance dispersal events; for example molecular clock analyses of a number of genera suggest that their contemporary diversity arose within the last 5 million years e.g. Plantago (Tay et al., 2010), Ourisia (Meudt et al., 2009), Myosotis (Winkworth et al., 2002a) and Ranunculus (Lockhart et al., 2001). The past 5 million years was a period of rapid geological uplift and numerous climatic oscillations in New Zealand, leading to many different situations that could influence speciation such as adaptation to new habitats after the uplift of the Southern Alps (Haase et al., 2007; Lockhart et al., 2001; Trewick and Morgan-Richards, 2005; Wagstaff and Garnock-Jones, 1998), and climate fluctuations (Raven, 1973; Winkworth et al., 2002b). Mountain building has obvious implications for species formation, in terms of both creating new habitats and creating barriers to gene flow, consequently promoting the evolution of local variants. Frequent climatic fluctuations are also hypothesised to affect the radiations of plant lineages in that climate change produces new habitats that allow range expansion and provide opportunities for the differentiation of local morphological or 
ecological variants. Ongoing climate change can also lead to range contraction, which may result in the formation of additional local variants either by local differentiation or perhaps hybridisation (Winkworth et al., 2005).

Several cycles of this pattern (such as repeated glacial cycles) might be expected to produce many variants, especially if migration paths result in hybridisation or introgression between previously isolated forms (Winkworth et al., 2005). This model of species diversification would be expected to result in considerable mixing of genotypes, and therefore complex patterns of phylogenetic relationships. Recent molecular phylogenetic studies on New Zealand plants support this suggestion (Winkworth et al., 2005). Several studies have found complex patterns of relationships, using chloroplast markers for example (e.g. Veronica (as Chionohebe) (Meudt and Bayly, 2008) and Leucogenes (Smissen and Breitwieser, 2008). Additionally, species with conspicuous morphological and ecological differences exhibit little or no genetic differentiation with both nuclear and chloroplast loci, suggesting that morphological differentiation has occurred over a relatively short period of time. Winkworth et al. (1999) report that in the New Zealand flora a small amount of genetic change in neutral and cpDNA markers can underlie dramatic morphological differences in recently evolved plant species. The southern hemisphere members of Myosotis have very little genetic diversity (measured with the matK and ITS loci) compared to northern hemisphere representatives, yet they displayed greater morphological variation. Speciation in New Zealand plant groups may also have followed displacement along the alpine fault (Haase et al., 2007). Cook Strait, which separates the North and South Islands of New Zealand, currently acts as a barrier as well, although there may have been land bridges connecting the North and South Islands during the glacial cycles of the Pliocene (Lewis et al., 1994).

\section{Molecular phylogenetics}

Molecular phylogenetics has been increasingly used as a means of clarifying evolutionary histories and refining taxonomy when traditional methods, such as morphological data and fossil records, are ambiguous. The true evolutionary history of a given set of sequences is rarely known, and so phylogenetic trees represent 
estimates of 'true trees' (Nei and Kumar, 2000). Phylogenies treat speciation as a dichotomously branching event, an assumption that is frequently violated especially in recently evolving lineages where hybrisation and incomplete lineage sorting can confuse the picture (Woolley et al., 2008).

Hybridisation, as either homoploid hybrid speciation or allopolyploidy, has contributed extensively to angiosperm diversity. Whereas allopatric speciation via vicariance or long distance dispersal and subsequent establishment may be responsible for most speciation events, the evidence for hybridisation is nevertheless widespread (Soltis and Soltis, 2009). Allopolyploidy (hybridisation followed by genome duplication) allows instant speciation when one or several polyploid hybrids arise sympatrically with one (or both) of their parent populations (Petit et al., 1999). Allopolyploidy also leads to rapid diversification due to 'genomic shock' that can lead to massive genome restructuring. Because of genetic and genomic changes, individuals may arise with a modified phenotype and ecological preferences and hence are able to exploit new niches or to out compete progenitor species (Soltis and Soltis, 2009). Hybridisation and polyploidy are common in many New Zealand plant groups (Lockhart et al., 2001; Morgan-Richards et al., 2009; Wagstaff et al., 2010). Hybridisation can be a confounding factor in phylogenetic reconstruction, leading to reticulation, which cannot be displayed on a dichotomously branching tree (Woolley et al., 2008). Alternative methods such as networks can prove useful in these instances. Reconstructing phylogenetic trees from independent sources such as chloroplast and nuclear DNA is also a useful method for exploring whether or not hybridisation has occurred because conflict between loci from the two different genomes can implicate hybridisation (although it can also implicate incomplete lineage sorting, or that the markers in question are inappropriate).

As well as the assumption of dichotomous speciation, molecular phylogenetic inference relies on the selection of useful genetic markers, assumption of correct alignment of sequences, selection of a model of evolution and the choice of tree building method. Tree building methods used in this thesis include maximum parsimony, maximum likelihood and Bayesian approaches. Under parsimony, the preferred phylogenetic tree is the tree that requires the least evolutionary change to explain some observed data. The maximum-likelihood method takes a model of 
sequence evolution (essentially a set of parameters that describe the pattern of substitutions) and searches for the combination of parameter values that gives the greatest probability of obtaining the observed sequences. The Bayesian approach selects the tree that has the greatest posterior probability (the probability that the tree is correct), under a specific model of substitution (Bromham and Penny, 2003). In plants, genetic markers commonly used for phylogenetic analysis include chloroplast DNA and nuclear DNA (and to a lesser extent mitochondrial DNA), though DNA fingerprinting methods such as amplified fragment length polymorphisms (AFLPs) have been gaining in popularity, especially when DNA sequence markers are not variable enough for such purposes.

\section{Choosing DNA markers for molecular phylogenetic study}

Choosing which genetic markers to use is an important process when attempting to reconstruct molecular phylogenies. Ideally, markers are chosen based on their ability to differentiate between taxa at the level that the research requires. Different molecular tools are required for different questions, because of varying rates of sequence evolution among genomes, genes and gene regions (Small et al., 2004) and the probability that historical signal may be swamped by more recent noise in rapidly-evolving sequences. There is a vast array of molecular tools available e.g. DNA or amino acid sequencing, micro-satellites and AFLPs. The plant systematics community is currently using only a small fraction of tools on hand, with the vast majority of studies considering either chloroplast DNA (cpDNA) or nuclear ribosomal DNA (rDNA).

\section{Organellar DNA (chloroplast and mitochondrial)}

One advantage of cpDNA as a tool in molecular systematics is its relatively simple genome. Chloroplast genomes vary little in size, structure and gene content over all angiosperms. The genome typically ranges in size from $135 \mathrm{~kb}$ to $160 \mathrm{~kb}$ and is characterized by a large (ca. $25 \mathrm{~kb}$ ) inverted repeat which divides the remainder of the genome into one large and one small single copy region (Wolfe and Randle, 2004). This conservatism, and that the chloroplast genome has been sequenced in its entirety for a number of genomes, means it is possible to develop universal primers (Wolfe and Randle, 2004). The large number of copies in each cell (there may be as 
many as 900 cpDNA molecules per chloroplast and 50000 per cell (Kelchner, 2000), allows for easy extraction and amplification (Cowan et al., 2006). Additionally, organelles are usually inherited uni-parentally, which potentially results in more straightforward interpretation (Wolfe and Randle, 2004).

Ironically the properties that make cpDNA an attractive tool for molecular systematics also hinder its usefulness in phylogenetic analysis: phylogenies based on chloroplast DNA may not reveal the true evolutionary history of a taxon for a number of reasons. Because cpDNA is uni-parentally inherited and haploid, it reveals only half of the parentage in plants of hybrid or polyploid origin (Small et al., 2004). Chloroplast DNA alone is unable to identify phylogenetic conflicts arising from hybrid ancestry.

An additional limitation of cpDNA in molecular phylogenetic studies is its relatively slow rate of evolution, which means that even non-coding cpDNA regions often fail to resolve phylogenies at low taxonomic levels. It is for this reason that mitochondrial DNA is rarely used in plant systematics studies (Ennos et al., 1999). However, an unusually high rate of mtDNA evolution has recently been reported in Plantago (Cho et al., 2004) and several other plant groups such as Silene (Houliston and Olson, 2006) and Geraniaceae (Bakker et al., 2006). Twelve chloroplast and two mitochondrial markers are trialed in this study (see Table 3.1), and two chloroplast markers are selected for use ( $\operatorname{trn} L-F$ and $\operatorname{trn} K)$.

\section{Nuclear ribosomal DNA}

Nuclear ribosomal DNA has been widely used in order to complement organellar DNA by obtaining additional, independent, bi-parentally inherited phylogenetic estimates that evolve at a much faster rate. The internal transcribed spacer (ITS) region, found in the nuclear genome of plants, fungi and animals is now the most commonly used marker in plant systematics (Alvarez and Wendel, 2003). This refers to the region of $18 \mathrm{~S}, 5.8 \mathrm{~S}$ and $26 \mathrm{~S}$ ribosomal genes, along with two internal transcribed spacers. Ribosomal genes exist in tandem arrays of genes composed of hundreds to thousands of copies per array (Small et al., 2004). The ITS region is easily amplified with universal primers due to its conserved priming sites (Hillis and 
Dixon, 1991), which flank non-coding regions with high genetic variation in most groups (Alvarez and Wendel, 2003). As a consequence of repeated use, there is a wide assortment of ITS sequences readily available on Genbank (http://www.ncbi.nlm.nih.gov).

There are a number of concerns with using ITS as a marker, especially if it is the only marker used in a study. One concern is the high level of sequence variation, which can lead to difficulties in aligning, potentially introducing homoplasy, especially when including outgroups (Alvarez and Wendel, 2003). The biggest concern however is that the copies of ribosomal genes may evolve separately, leading to sequence variants within and between arrays. Individuals included in phylogenetic studies may therefore possess a multiplicity of sequence types. This is a problem if pseudogenes are preferentially amplified over functional rDNA, which can result in incorrect inference of relationships between samples (Alvarez and Wendel, 2003). Cloning all sequence variants may overcome this problem, but there is still a chance that not all variants will have been amplified. Despite these concerns ITS is used in the present study, partly due to the relative ease of sequencing it and the high level of variation it contains, but mainly for the purpose of linking in with previous research (thus continuing the bandwagon effect described in Alvarez and Wendel (2003)). The use of low-copy nuclear genes, still relatively new to the field, may provide a useful alternative (Hughes et al., 2006; Small et al., 2004; Steele et al., 2008)

\section{Low-copy nuclear markers}

The nuclear genome of angiosperms contains a large number of potential genes for phylogenetic analysis - predicted to be from 26,500 nuclear genes in Arabidopsis thaliana to 41,000 in Oryza sativa (Sterck et al., 2007). As with rDNA, there may be problems with distinguishing between orthologous and paralogous gene copies, but this can usually be overcome by sequencing an adequate number of clones (Steele et al., 2008). The main advantages of low-copy nuclear genes include the higher rate of evolution and the opportunity to accumulate data sets from multiple, unlinked loci. The drawbacks are the lack of universal primers, which means a greater investment in extensive screening is required to identify the most promising loci (Hughes et al., 
2006). As there is no a priori reason to expect that any particular gene or gene family will be useful at any given phylogenetic depth for any given group of plants it can be difficult to know where to start looking for a useful gene to sequence (Small et al., 2004). There are several solutions to this. Some researchers prefer to use genes that have proven phylogenetically useful in the past, others conduct screens of unknown genes. Padolina (2006) took a computational approach to design universal angiosperm primers by querying the MoBIoS database (Miranker et al., 2003) to compare the genomes of the monocot $O$. sativa and the eudicot $A$. thaliana to search for primer combinations that occurred only once in each of the two genomes. This search (with additional criteria described in Padolina (2006)) resulted in 141 primer combinations available for any researcher to trial on their own plant group of interest (available at http://www.sbs.utexas.edu/roxisteele/). Steele et al. (2008) further trialed these primers and selected 32 primer combinations that amplified regions in representatives of at least two out of five distantly related angiosperm families. It was outside the scope of the present study to trial all 32 suggested primers, but nevertheless I selected four of these primers to trial based on their success in amplifying across the highest number of families in Steele et al.'s (2008) study. I also trialed an additional three low-copy nuclear DNA loci selected from other published studies (Levin et al., 2009; Li et al., 2008; Peralta and Spooner, 2001), however none of these were chosen for further analysis in the present study.

Corroboration of phylogenetic hypotheses by independent datasets increases confidence in a given tree. Sequences can be used in combination with each other if their gene histories do not conflict (Cummings et al., 1995). Incongruence between datasets can be of use to allow visualization of processes such as hybridisation, introgression, reticulation and incomplete lineage sorting if these have occurred in the past (Vriesendorp and Bakker, 2005). For events such as hybridisation, parental lineages may also be revealed based on the phylogenies of the different markers e.g. within the Gnaphalieae (Smissen et al., 2004).

\section{AFLPS}

When both cpDNA and ITS sequencing fail to resolve phylogenies, the amplified fragment length polymorphism (AFLP) approach has the potential to overcome such 
difficulties. AFLPs are generated by complete restriction endonuclease digestion of total genomic DNA, followed by selective PCR amplification and electrophoresis of a subset of the fragments, resulting in a unique, reproducible fingerprint (or profile) for each individual (Meudt and Clarke, 2007). AFLP markers are sampled throughout the genome and they can therefore reveal rare genetic differences in groups with low sequence variations e.g. among closely related species, crop species, or at the intraspecific level (Meudt and Clarke, 2007). This simultaneous analysis of many loci representing the whole genome is also more likely to generate a true species tree, rather than generating a particular gene tree as sequencing an individual locus is more prone to do (Després et al., 2003). AFLPs have not been widely used in phylogenetic studies though, and instead have been more frequently used to delimit species using ordination methods. Some consider AFLP data inappropriate for any kind of phylogenetic study e.g. (Kosman and Leonard, 2005) but others have found useful phylogenetic signal in AFLP datasets (Koopman, 2005; Meudt and Clarke, 2007). For AFLPs to be suitable for phylogenetic analysis there are two requirements: fragments must have evolved independently, and fragments of equal length must be homologous (Koopman, 2005). There is very little study into the independent evolution of the fragments, but several people have explored the homology or otherwise of same-sized fragments, and have found that in general the more closely related two samples are the more similar the sequences (and therefore homology) of same sized fragments. It is difficult to universally apply these results as taxonomic rank is not equivalent across groups (Meudt and Clarke, 2007), but Koopman et al. (2008) estimated that AFLPs are able to recover phylogenetic relationships among plant samples if the nrITS divergence is between 10-30 nucleotides between sequences. AFLPs have proven useful for exploring variation in the New Zealand flora in a number of groups including Ourisia (Meudt et al., 2009), Pseudopanax, (Perrie and Shepherd, 2009), and Leucogenes (Smissen and Breitwieser, 2008) and I apply them here to New Zealand Wahlenbergia.

\section{Molecular clocks}

The molecular clock arose from the observation that the amount of difference between the DNA of two species is a function of the time since their evolutionary separation. This provides a universal tool for placing past evolutionary events in time 
(Bromham and Penny, 2003). Since their first conception, molecular clock dating methods have rapidly increased in sophistication and reliability. When first proposed it was necessary to assume a constant rate of evolution throughout the tree, in order to infer divergence dates (the strict molecular clock). This practice has long been challenged by results from datasets showing considerable departures from clocklike evolution (Britten, 1986) and when rate variation does exist among lineages it can seriously mislead not only divergence date estimation but also phylogenetic inference (Drummond et al., 2006). Recent developments allowing for a relaxed molecular clock algorithm, which can concurrently estimate the phylogeny and the divergence rate with different rates of evolution in certain lineages, has vastly improved the accuracy of molecular clock estimates (Drummond et al., 2006). To use genetic distance to estimate divergence time a calibration rate is needed that states the amount of genetic change expected per unit of evolutionary time. The calibration rate is usually calculated for each data set using a known date of divergence to estimate the rate for the whole phylogeny (Bromham and Penny, 2003). The known date is most commonly taken from the fossil record, but other sources include biogeography (for example, the formation of an island). The choice of calibration date is crucial to the accuracy of molecular dates, as shown by the difference in date estimates from studies using the same data and methods but different calibration dates e.g. dating the Cambrian explosion (Smith and Peterson, 2002). In this thesis a relaxed molecular clock using a Bayesian algorithm as implemented in BEAST (Drummond and Rambaut, 2007) is used. The Bayesian algorithm has its strengths and weaknesses; but the explicit nature of the Bayesian method makes its flaws obvious and makes it relatively easy to determine which assumptions should be examined in more detail (Sanderson et al., 2004).

\section{Introduction to study group}

In this thesis the phylogeny of the New Zealand species of Wahlenbergia Schrader ex Roth (Campanulaceae) and their relationships with overseas Wahlenbergia are explored using DNA sequencing and DNA fingerprinting data. Wahlenbergia is a large genus (ca. 260 spp.) with a mainly southern hemisphere distribution. The centre of diversity is South Africa (ca. 150-200 spp.), with a secondary centre in Australasia 
(ca. 36 spp.), although the genus is also indigenous to New Caledonia, New Guinea, Malaysia, Japan, Taiwan, China, India, Europe, South America and some of the small volcanic Pacific islands e.g. Juan Fernández and Tonga (Petterson, 1997b). The name Wahlenbergia was first introduced by Schrader (1814) but was invalidly published (van Steenis, 1960), which means the first validly published description was by Roth (1821). Cervicinia (Delile, 1813) is an earlier validly published name for the genus, but the later Wahlenbergia has been conserved against it (Smith, 1992). The type is a South African individual of $W$. capensis (van Steenis, 1960), a South African species that has subsequently been introduced into Australia (Smith, 1992).

Wahlenbergia belongs to the large, almost cosmopolitan angiosperm family, Campanulaceae. When considered in the strict sense (excluding Cyphiaceae, Cyphocarpaceae, Lobeliaceae, and Nemacladaceae, which are sometimes treated as part of the Campanulaceae (Haberle et al., 2009)) several recent studies have confirmed the family's monophyly, using morphological (Gustafsson and Bremer, 1995) and molecular data (Lundberg and Bremer, 2003). In their summary of the taxonomic history of the family, Haberle et al. (2009) noted that the first comprehensive monograph of the family was published by De Candolle in 1830 with 21 genera and 234 species. De Candolle divided the family into two groups based primarily on capsule dehiscence: Campanuleae, which dehisce by lateral pores or valves, and Wahlenbergieae, which dehisce by apical valves or pores. The largest genus in the family is the mainly northern hemisphere Campanula (ca. 421 species), which forms a broad geographic and taxonomic complement to the second largest (and mostly southern hemisphere) genus, Wahlenbergia (Smith, 1992). Haberle et al. (2009) further note that later authors have added to and altered De Candolle's subfamilial divisions, for example Schönland (1889) recognized three main family subdivisions based on capsule dehiscence and the position of ovary locules in relation to the calyx lobes. These were: Campanulinae (having carpels that are generally opposite the calyx lobes, with laterally dehiscent capsules), Wahlenberginae (sepals generally opposite the carpels, but chiefly apically dehiscent capsules) and the Platycodoninae (carpels alternate with the lobes, and mostly apically dehiscent capsules). 
More recent phylogenetic studies have again (though informally) revised the subfamilial names within the Campanulaceae, for example Eddie (2003) and Haberle et al. (2009) use the term "campanuloid" to refer to the mostly northern hemisphere taxa allied with Campanula, "wahlenbergioid" to refer to the predominantly southern hemisphere taxa traditionally associated with Wahlenbergia, and "platycodonoid" for the chiefly Asian colporate/colpate taxa. This thesis will follow the informal subfamilial nomenclature of Eddie (2003) and Haberle et al. (2009). At lower taxonomic levels, the delineation of genera and species in the family has varied widely among authors, who have subdivided the family into 35 to 55 genera and up to 1,046 species (Haberle et al., 2009).
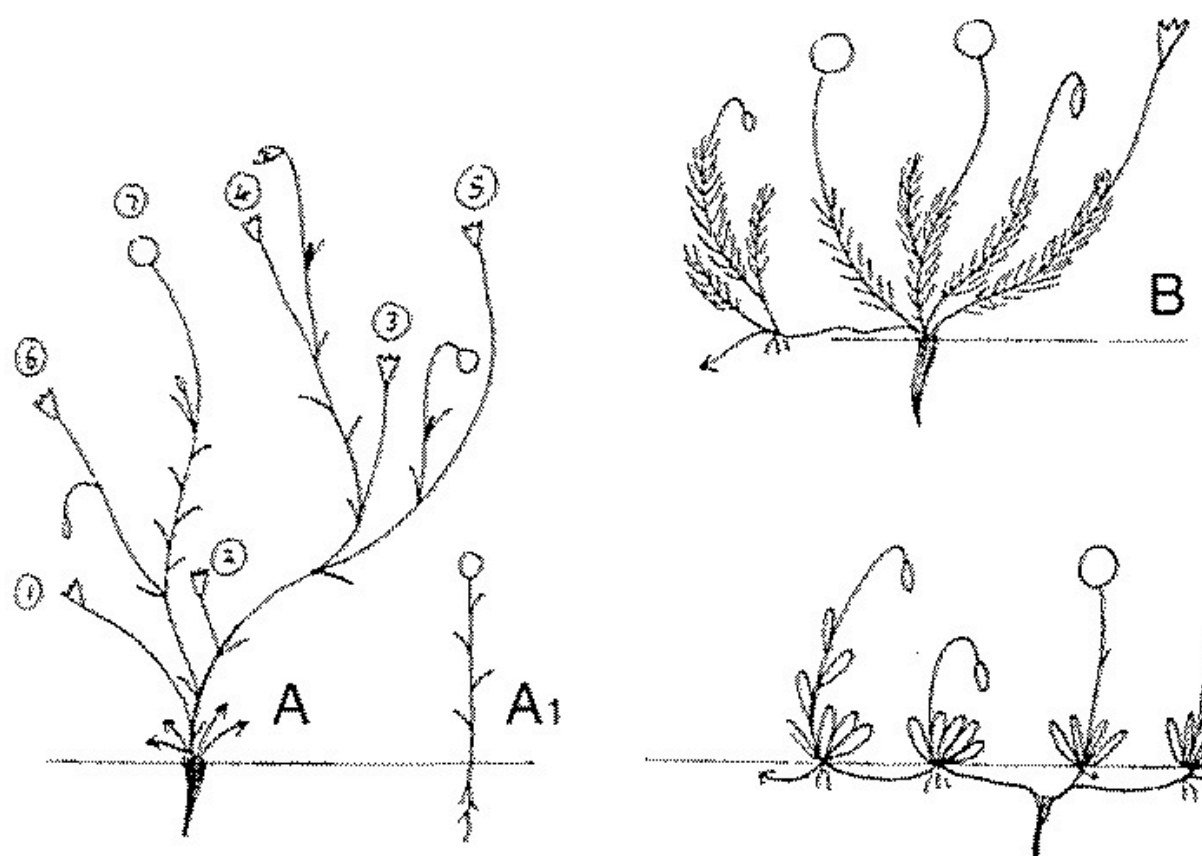

Figure 1.1 Growth forms of New Zealand Wahlenbergia. $\mathrm{A}=$ radicate growth form, numbers indicate order of flowering, $\mathrm{A} 1=$ false annuals, $\mathrm{B}=$ suffructose rhizomatous and $C=$ creeping rhizomatous. Drawing from Petterson (1997b).

New Zealand Wahlenbergia plants are all herbaceous perennials (Petterson, 1997b), though annuals are common overseas (e.g. W. gracilenta in Australia) and shrubby Wahlenbergia occur in South Africa and on islands such as Juan Fernández and Ascension (Smith, 1992). Plants in this genus are generally insect pollinated (Petterson, 1997b) and are conspicuously protandrous - pollen is shed onto the pollen-presenting stylar hairs while the flower is in bud, and these hairs then collapse 
soon after the flower is open (Lloyd and Yates, 1982). The stigma of the same flower then becomes receptive (Smith, 1992).

A number of chromosome counts have been undertaken on several Wahlenbergia species. Throughout Australasia and southern Asia, the genus has the base chromosome number of $x=9$, though many ploidy levels have been found, e.g., $2 \mathrm{n}=2 \mathrm{x}=18,2 \mathrm{n}=4 \mathrm{x}=36,2 \mathrm{n}=6 \mathrm{x}=54,2 \mathrm{n}=8 \mathrm{x}=72$, and $2 \mathrm{n}=10 \mathrm{x}=90$ (Petterson et al., 1995; Smith, 1992). In New Zealand three ploidy levels have been reported $2 n=36,54$ and 72 (Petterson et al., 1995), which correspond to different growth forms (Figure 1.1). In contrast, chromosome numbers in Africa have been reported with base chromosome numbers of $x=7,8$ and 9, with only one example of a polyploid (Smith 1992). Recent research into Wahlenbergia has focused on identifying new species (Plunkett et al., 2009) assessing their use as indicators of climate change (Gallagher et al., 2009), exploring their palatability (Odhav et al., 2007), their seed longevity (Kochanek et al., 2009) and their part in floral deception (Peter and Johnson, 2008).

No phylogenetic studies of the Australasian Wahlenbergia have been undertaken so far. Cupido (2009) has recently conducted a molecular systematic study of the South African Campanulaceae using ITS and trnL-F in which he included 53 Wahlenbergia samples. One recent molecular phylogenetic study of the Campanulaceae family as a whole has found Wahlenbergia to be polyphyletic (Haberle et al., 2009) though that study included few Wahlenbergia samples. Haberle et al. (2009) used three chloroplast genes - atpB, $r b c L$ and $m a t K$ to reconstruct a phylogeny of the Campanulaceae that included 102 taxa from 41 genera. They included five Wahlenbergia species, two from St Helena Island, one from Juan Fernández Island, one from Australia and one from Europe. These showed Wahlenbergia to be polyphyletic as they were recovered in three separate clades, the European sample ( $W$. hederacea) not even grouping with the wahlenbergioids.

\section{Brief taxonomic history of Wahlenbergia in New Zealand}

In this thesis, I focus on the evolution of Wahlenbergia species that are native to New Zealand. The current Wahlenbergia taxonomy in New Zealand is mostly based on a recent morphological revision by Judith Petterson (nee Hay) (1997b). In that 


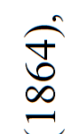

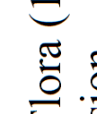

한:

政

ริำ

No

产

을

혼

홍ํำ

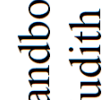

政

थ

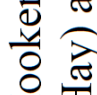

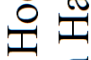

$\Xi \Xi$

寻

ปี

$\frac{2}{\frac{2}{3}} \overline{0}$

$\approx$

范

壱亚

를

乙。

몽

$4 \cdot \frac{\pi}{5}$

苛

离

을

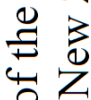

䃞

疅

=

童产

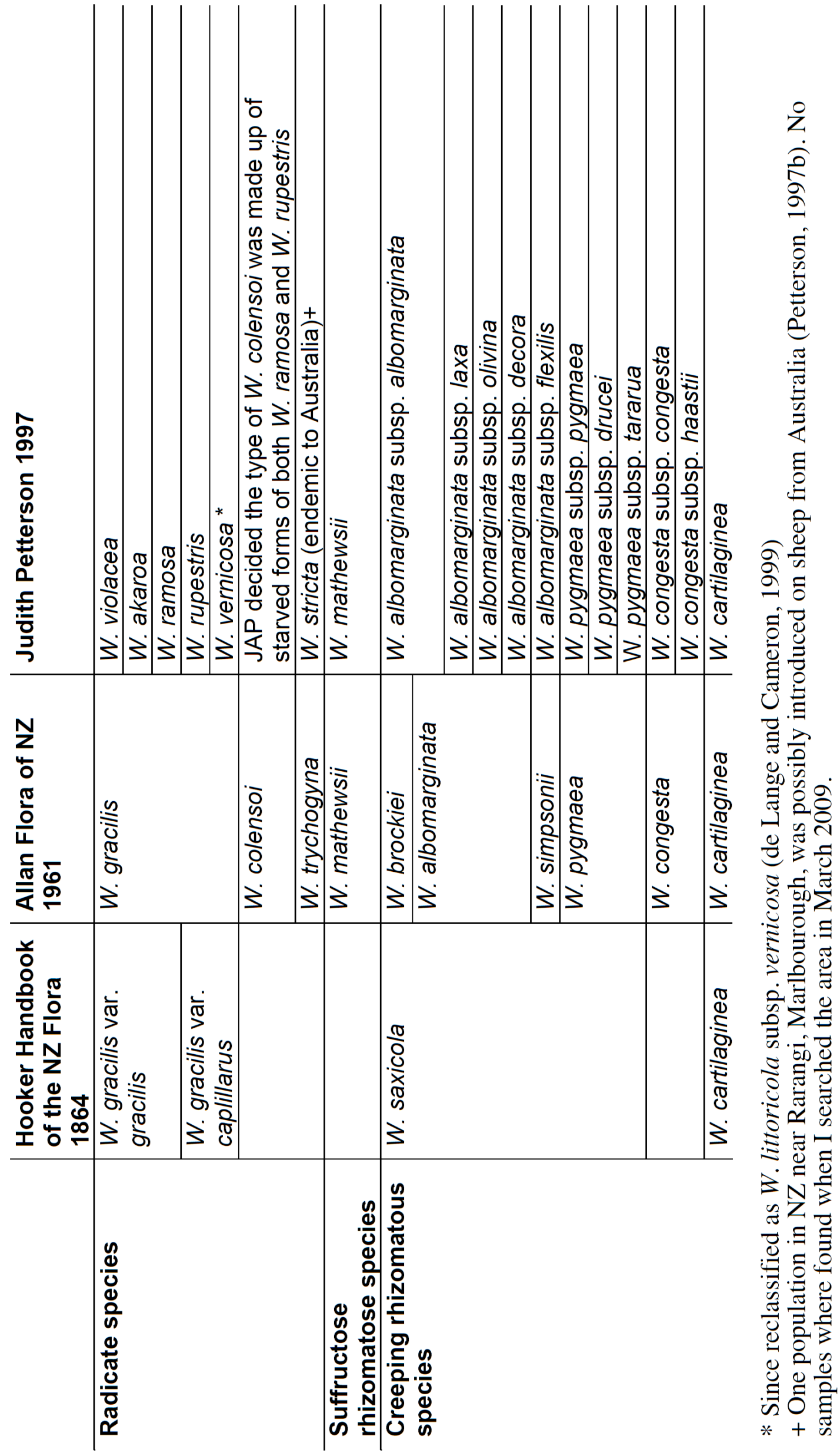


revision she provided a taxonomic treatment for nine endemic and one indigenous species (also found on the Kermadecs and possibly Tonga), with a further seven endemic subspecies (Table 1.1). The New Zealand Wahlenbergia can be separated into four convenient groups based on growth form and chromosome number. These groups are temperate radicate $(2 \mathrm{n}=72,4$ species $)$, subtropical radicate $(2 \mathrm{n}=54,1$ species), suffructose rhizomatous ( $2 n=361$ species) and creeping rhizomatous ( $2 \mathrm{n}=36,4$ species) (Petterson, 1997b; Petterson et al., 1995). The only subsequent taxonomic change has been to reduce $W$. vernicosa to a subspecies of the Australian W. littoricola (de Lange and Cameron, 1999). Earlier taxonomic treatments for the New Zealand species (e.g. Hooker (1864) and Hay in Allan (1961)) included fewer (and different) species to Petterson, which is indicated in Table 1.1.

\section{New Zealand radicate species}

The five New Zealand radicate species all inhabit the lowland. They were originally named as one species - Campanula gracilis (Forster, 1786). There is a great deal of confusion over whether the type of this species is housed at Kew or Gottingen, and whether it is made up of specimens collected in New Zealand or New Calendonia (or both) (Nicolson and Fosberg, 2004). In 1913 (after the valid publication of Wahlenbergia and the change of genus from Campanula for the New Zealand species) N. E. Brown published Wahlenbergia colensoi, a species he based on small radicate Wahlenbergia specimens collected by William Colenso, and sent by him to Sir W. J. Hooker at Kew in the 1840s (Petterson, 1997a). Descriptions of two further radicate Wahlenbergia species, $W$. ramosa and $W$. rupestris were also published (Simpson, 1945, 1952). In her 1997 revision Petterson recognised 5 radicate species, none of which she called $W$. gracilis as she considered the type to have been collected in New Caledonia, and thought that none of the New Zealand Wahlenbergia were conspecific with the type (Petterson, 1997a). She retained both of Simpson's species ( $W$. ramosa and $W$. rupestris), but reduced $W$. colensoi to a synonym of both, having decided that it represented 'starved forms' of the white flowered $W$. ramosa and $W$. rupestris (Petterson, 1997a). She described three new species, $W$. akaroa, $W$. violacea and $W$. vernicosa. The subtropical $W$. vernicosa has a different chromosome count $(2 n=54)$ relative to the other New Zealand radicates. The other four temperate radicate species ( $W$. violacea, $W$. akaroa, $W$. ramosa and 

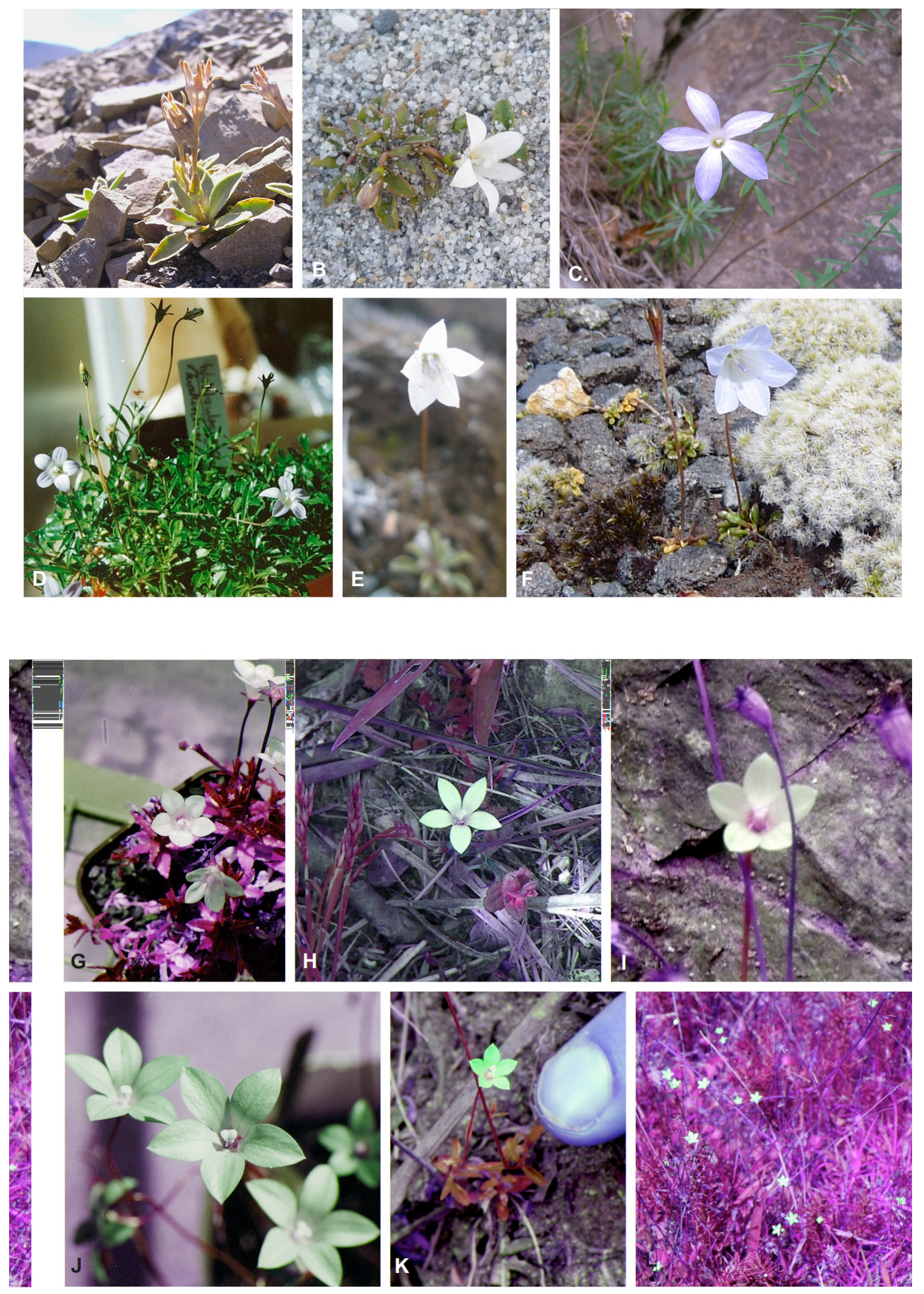

Figure 1.2 New Zealand Wahlenbergia. A-F have the rhizomatous growth form. $\mathrm{A}=W$. cartilaginea, $\mathrm{B}=W$. congesta subsp. haastii, $\mathrm{C}=W$. matthewsii (suffructose rhizomatous growth form), $\mathrm{D}=W$. pygmaea subsp. pygmaea, $\mathrm{E}=W$. albomarginata subsp. olivina, $\mathrm{F}=W$. albomarginata subsp. albomarginata. G-L have the radicate growth form. $\mathrm{G}=W$. vernicosa. $\mathrm{H}-\mathrm{L}$ illustrate the $W$. gracilis complex. $\mathrm{H}=W$. rupestris, $\mathrm{I}=W$. ramosa, $\mathrm{J}=W$. akaroa, $\mathrm{K}$ and $\mathrm{L}=W$. violacea. Photos A, C, F and H C P. Garnock-Jones, B, E, I, K and L (C J. Prebble and $\mathrm{D}, \mathrm{G}$ and $\mathrm{J}$ (C) G. Petterson. 
$W$. rupestris) share a chromosome number of $2 n=72$, and have since been referred to as a single species: Wahlenbergia gracilis (Webb and Simpson, 2001), hereafter referred to as the ' $W$. gracilis complex'. Although there is a certain amount of morphological variation within the $W$. gracilis complex (see figure 1.2) it is difficult to find characters that consistently distinguish different lineages within this group.

\section{New Zealand rhizomatous species}

The three most morphologically distinct rhizomatous New Zealand Wahlenbergia have the simplest taxonomic histories. They were each named once, and these names have been retained until the present ( $W$. cartilaginea, $W$. congesta and the suffructose $W$. matthewsii see Figure 1.2). The other rhizomatous Wahlenbergia in New Zealand have a more involved taxonomic history. Originally, they were referred to as Campanula saxicola, now known as Wahlenbergia saxicola and considered endemic to Tasmania. Despite the valid publication of $W$. albomarginata in 1852, alpine Wahlenbergia in New Zealand continued to be referred to W. saxicola for some time (e.g. see Table 1.1). The name $W$. pygmaea Colenso was then published in 1899 to describe small alpine rhizomatous Wahlenbergia. These are the two species recognized by Petterson (1997b). Four further species in this complex were described in the $20^{\text {th }}$ century. Petterson (1997b) considered two to be synonymous ( $W$. flexilis and $W$. simpsonii) and reduced them both to a subspecies of $W$. albomarginata ( $W$. albomarginata subsp. flexilis); another ( $W$. laxa) was also reduced to a subspecies of $W$. albomarginata and the fourth ( $W$. brockiei) she considered to be synonymous with $W$. albomarginata.

\section{A note on species concepts}

There has been much discussion in recent times about different species concepts, with vast numbers of different definitions leading to different conclusions concerning the boundaries and number of species (De Queiroz, 2007). Mayer (1997) listed 24 differently named species concepts, the most well-known being the biological (Mayr, 1942), ecological, evolutionary and phylogenetic concepts. While these concepts differ in their methods for delimiting species, they share the common idea that species are separately evolving metapopulation lineages (the 'unified species 
concept' of De Queiroz (2007) and it is essentially a confusion between detection protocols and concepts that has lead to the differences in opinion (Hey, 2006). De Queiroz (2007) demonstrates that the different concepts (e.g. biological, ecological, phylogenetic) are better thought of as subcategories, and that evidence from different lines of investigation can be brought to identify the same speciation event. Indeed, a highly supported hypothesis of lineage divergence (i.e. speciation) requires evidence from more than one line of investigation. However, the absence of evidence for speciation from any one or more of the lines of investigation does not constitute evidence against a hypothesis of lineage separation, as the lineage simply may have not yet evolved the property in question. This is particularly pertinent in recently evolving lineages, which is where most of the controversy in delimiting species arises (De Queiroz, 2007; Hey, 2006; Shaffer and Thomson, 2007). Both Smith (1992) and Petterson (1997b) followed a morphological species concept in their revisions of Wahlenbergia, though neither were explicit about levels of morphological differentiation used to delimit species or subspecies. The unified species concept will be adopted in this study, with the central line of investigation involving monophyly at one or multiple DNA loci, coupled with morphological, chromosomal and/or ecological distinctness.

\section{Study aims}

The overall aim of this Masters project is to explore the evolution of the Australasian Wahlenbergia at a number of different scales. At the large scale I will undertake a molecular phylogenetic analysis exploring the biogeography of Wahlenbergia including samples from South Africa, Europe, Australia and New Zealand. At the smaller scale I will assess the current taxonomy of the New Zealand Wahlenbergia, using analysis of DNA sequencing and AFLP markers. 


\title{
Chapter 2: Biogeography and a first phylogeny of Wahlenbergia (Campanulaceae)
}

\begin{abstract}
The first phylogeny of Wahlenbergia was reconstructed using about $20 \%$ of the genus based on the nuclear ribosomal ITS marker and the chloroplast $\operatorname{trn} L-F$ marker with samples from South Africa, Europe, Australia and New Zealand. Wahlenbergia was confirmed to be polyphyletic, though most of the species form a clade. Wahlenbergia originated in South Africa about 16.2 mya, then dispersed to Australasia and radiated into 45 species and subspecies about 3.7 mya, thus refuting the hypothesis of Gondwanan vicariance for the Australasian group. Two dispersals from Australia to New Zealand are hypothesised, one leading to a radiation of species with the rhizomatous herbaceous growth from ca. 1.0 mya and the other leading to a radiation of species with the radicate growth form 0.49 mya. Dispersals from Australia to New Zealand match the expected direction, following the west wind drift and ocean currents. An herbaceous growth form was shown to be ancestral for the genus as a whole, and chromosome levels have increased as the genus has evolved in Australasia.
\end{abstract}

\section{Introduction}

The genus Wahlenbergia is one of the largest genera within the Campanulaceae, comprising upwards of 260 species (Haberle et al., 2009) mainly distributed in the southern hemisphere. The centre of diversity is South Africa, with a secondary centre in Australasia (Petterson, 1997b; Smith, 1992) although the genus is also indigenous to New Caledonia, New Guinea, Malaysia, Japan, Taiwan, China, India, Europe, South America and some of the small volcanic Pacific islands, e.g., Juan Fernández 
and Tonga (Petterson, 1997b). There are no Wahlenbergia native to North America. The majority of Wahlenbergia species are annual or perennial herbs, though a few species have a shrubby habit (Smith, 1992). Their flowers have five petals and range in colour from white to blue-purple and are generally insect pollinated (Petterson, 1997b). Their seeds are light and are dispersed by wind (Thorsen et al., 2009). The aim of this research is to produce the first phylogeny of Wahlenbergia to determine whether Wahlenbergia is monophyletic, whether the South African, Australian and New Zealand Wahlenbergia are reciprocally monophyletic, and to establish when the South African and Australasian Wahlenbergia are likely to have evolved.

Recent molecular studies into the phylogeny of Campanulaceae have included few samples of Wahlenbergia i.e. 3 samples in Roquet et al. (2009) and 5 samples in Haberle et al. (2009). Both of these studies found Wahlenbergia to be polyphyletic. In Haberle et al.'s (2009) study based on three chloroplast markers, the five Wahlenbergia samples were placed in three separate clades on their tree. Interestingly the Australian sample ( $W$. gloriosa) grouped with only one of the samples from St Helena Island ( $W$. linifolia). The other St Helena sample ( $W$. angustifolia) was sister to the sample from Juan Fernández ( $W$. berteroi) and the European sample grouped with no other Wahlenbergia, a relationship also found by Roquet et al. (2009). As the centre of diversity is South Africa, and the type of Wahlenbergia is a South African species (W. capensis) it is critical to understand the relationships between these peripheral species that have so far been included in phylogenetic studies and the core of the Wahlenbergia.

Recent studies of the New Zealand flora have used molecular phylogenetics to contrast two biogeographic scenarios: Gondwanan vicariance vs. Neogene dispersal, although these are two extremes in a range of potential hypotheses. Few studies support a vicariance scenario (e.g. Stöckler et al. (2002) for Agathis), whereas there is increasing evidence from molecular data that much of the biodiversity in New Zealand is the product of rapid evolution following relatively recent colonization (Winkworth et al., 2002a). This has led to much discussion over whether any of New Zealand has remained above water since splitting from Gondwanaland, or whether it has more recently (about 26 million years ago) surfaced after a submerged period termed the Oligocene drowning (Trewick et al., 2007). 
The current distribution of the southern hemisphere Wahlenbergia species could suggest a Gondwanan ancestry, with a few long distance dispersal events to Europe and Asia. However, a recent study into molecular dating of the Campanulaceae, which included three Wahlenbergia samples, dated the split between the "platycodonoid" species and the ancestor of the "campanuloid" and "wahlenbergioid" clades to between 36.1 and 41 million years ago (mya) (Roquet et al., 2009). These informal terms for groups within the Campanulaceae ("platycodonoid", "campanuloid" and "wahlenbergioid") were coined by Eddie et al. (2003) and are roughly equivalent to the subfamily terms used in Roquet et al. (2009). The dates from Roquet et al. (2009) are significantly later than the split up of Gondwanaland, and the absence of extant campanuloid species in Australasia suggests that long distance dispersal rather than vicariance is the most parsimonious explanation for the presence of Wahlenbergia there.

Roquet et al. (2009) used two chloroplast markers ( $r b c L$ and $\operatorname{trn} L-F$ ) and set their molecular clock using four calibration points. First, they placed a minimum age of 16 mya on Campanula and related genera based on a Campanula seed fossil. Second, they used the endemism of Azorina vidalii (Campanulaceae) to the "island" Punta Delgada (which is actually a city on Santa Maria Island) in the Azores, which was formed 8 mya (Abdel-Monem et al., 1975), to date the split of this species from its sister species. Third, the endemism of Musschia aurea (Campanulaceae) to Madeira, dated as being no more than 5.2 mya, gave a date for the split of $M$. aurea from its sister species. Finally, they set a maximum age of 41 mya for the root node based on a large-scale dating analysis of the angiosperm families (Wikström et al., 2001).

Another recent study involving molecular dating analysis of the Campanulaceae included five Wahlenbergia samples, but the focus was on Cretan Campanula species (Cellinese et al., 2009). This study used three chloroplast loci and was calibrated at two points but did not report any dates regarding the Wahlenbergia clades. First, they calibrated the molecular clock using the same fossil seed as (Roquet et al., 2009) but, by making more assumptions about the closest living relatives of the seed fossil they placed it at a different node. Second, they also dated the root of the tree, but they set the age of the split between Campanulaceae and 
Lobeliaceae to between 45 mya and 80 mya, with this earlier date representing the age of the split between the Rousseaceae and the lineage that leads to Campanulaceae/Lobeliaceae (Wikström et al., 2003). They do not report their estimate for the age of the split between the subfamily Platycodoneae and the ancestor of the subfamilies Campanuleae and Wahlenbergieae (equivalent to the platicodonoid, campanuloid and wahlenbergioid of Roquet et al. (2009)), but their estimations are in general older than those of Roquet et al.'s (2009) study (compare Figure 4 in Cellinese et al. (2009) with Figure 3 in Roquet et al. (2009)).

The aims of this chapter are to: 1) produce the first phylogeny of Wahlenbergia including about $20 \%$ of the approximately 260 species in the genus, 2) date key events for Wahlenbergia with a focus on the Australasian and South African clades by extending the dating efforts of previous studies and including a larger group of Wahlenbergia samples, 3) map characters of interest onto the phylogeny, and 4) explore the biogeography of the genus and to test for evidence of either Gondwanan vicariance or long distance dispersal in the evolutionary history of this plant group in the southern hemisphere, with a particular focus on New Zealand.

\section{Materials and Methods}

\section{Genetic markers}

Chloroplast DNA (cpDNA) is widely used as a genetic marker - the chloroplast genome is a double stranded circular DNA molecule and has been sequenced in its entirety in a number of organisms, allowing for the development of universal primers (Wolfe and Randle, 2004). The $\operatorname{trn} L-F$ region comprises the $\operatorname{trn} L$ (UAA) intron, and an intergenic spacer between the $\operatorname{trnL}$ (UAA) 3' exon and $\operatorname{trnF}$ (GAA) (Taberlet et al., 1991). When it was first proposed as a cpDNA marker the intergenic spacer was considered potentially useful for evolutionary studies of related species and probably of populations of the same species (Taberlet et al., 1991). $\operatorname{trnL}-F$ has been used as a marker in Campanualceae molecular phylogenetic studies previously (Roquet et al., 2009) which makes it a practical choice in this case as outgroup samples are available on GenBank (http://www.ncbi.nlm.nih.gov/Genbank/). 
Nuclear ribosomal DNA has been widely used in order to complement organelle DNA as an additional, independent, bi-parentally inherited marker that evolves at a much higher rate. The internal transcribed spacer (ITS) region, found in the nuclear ribosomal genome of plants, fungi and animals is the most commonly used marker in plant systematics (Alvarez and Wendel, 2003). There are a number of concerns with using ITS as a marker, especially if it is the only marker used in a study. One concern is the high level of sequence variation that can occur among genera or even species, which can lead to difficulties in alignment, especially when including outgroups, which can potentially introduce homoplasy into the data set (Alvarez and Wendel, 2003). Like trnL-F, ITS has also been used in previous molecular phylogenetic studies of the Campanulaceae (Eddie et al., 2003).

\section{Study group}

Location of samples, collection details, voucher information, GenBank accession numbers and references are presented in Appendix 1. The study group comprised 75 samples, 58 of which were Wahlenbergia. Fifteen samples of New Zealand Wahlenbergia were included, which represented all ten native species. A selection of the Australian native Wahlenbergia (ten out of 27) and South African Wahlenbergia (31 out of ca. 150-200spp.) were also included. The South African samples were all recently sequenced by Chris Cupido (Cupido, 2009). An additional two European Wahlenbergia sequences downloaded from GenBank (http://www.ncbi.nlm.nih.gov/Genbank/) made up the ingroup. These represent all of the Wahlenbergia species currently sequenced for both ITS and trnL-F. Outgroup species were selected following Haberle et al. (2009) and some outgroup sequences were downloaded from GenBank. Name abbreviations (tag names) for ingroup taxa used in all figures and tables have the first four letters of the species or subspecies name in all caps, followed by the general location, followed by the specific locality (i.e. VIOL_SI_Dun is $W$. violacea from the Dun Mountains of the South Island of New Zealand). Tag names for outgroup taxa have the first four letters of the genus, followed by the first four letters of the species epithet (i.e., LOBE_CARD is Lobelia cardinalis; see Appendix 1 and figure legends for more details). 


\section{DNA extraction, amplification and sequencing}

Total DNA was extracted from leaf fragments dried in silica gel using a modification of the hot CTAB method of Doyle and Doyle (1990). Ground tissue from approximately $0.5-1.0 \mathrm{~cm}^{2}$ of sample leaf was transferred to a $1.5 \mathrm{~mL}$ disposable Eppendorf tube and incubated on a heat block for 45 minutes at $60^{\circ} \mathrm{C}$ in extraction buffer [100mK tris-HCL pH 8.0, $1.4 \mathrm{M} \mathrm{NaCl}_{2} 20$ mM EDTA, 2\% CTAB (hexadecetyltrimethylammonium bromide), 1\% PVP40 (polyvinylpyrrolidone)]. $0.6 \mathrm{~mL}$ chloroform was added and the solutions were gently mixed before spinning at 10000 rotations per minute (rpm) for 10 seconds. Following centrifugation the clear supernatant (upper phase) was transferred to a new Eppendorf tube and $0.6 \mathrm{~mL}$ of isopropenol was added before the solution was stood on ice for 5-10 minutes. If no DNA precipitation was obvious at this stage the extract solution was left overnight at $4^{\circ} \mathrm{C}$ to precipitate DNA. The following day the DNA precipitate was briefly spun (6000 rpm for 30 seconds) and transferred to a new Eppendorf tube containing 0.8 $\mathrm{mL} 80 \%$ ethanol using a cut pipette tip so as to avoid damaging the DNA. This wash step was repeated, followed by a final spin (6000 rpm 30-120 seconds) and the $80 \%$ ethanol was poured off. The DNA pallet was left to air dry, and then re-suspended in $50 \mu \mathrm{L}$ TE buffer (10mM Tris-HLC $\mathrm{pH}$ 8.0, 1mM EDTA).

Two regions were then amplified by polymerase chain reaction (PCR) using the following primer sequences: Chloroplast trnL-Ff:ATT TGA ACT GGT GAC ACG AG and trnL-Fc CGA AAT CGG TAG ACG CTA CG (Taberlet et al., 1991) and nuclear ribosomal ITS5: GGA AGT AAA AGT CGT AAC AAG AAG G and ITS28cc: CGC CGT TAC TAG GGG AAT CCT TGT AAG.

PCR amplification was performed in a Biometra $\mathrm{T}$ gradient machine (Whatman, Germany) in a final volume of $25 \mu \mathrm{L}$. Each $25 \mu \mathrm{L}$ volume contained $2 \mu \mathrm{L}$ of DNA template (nanodrop concentrations ranged from $20 \mathrm{ng} / \mu \mathrm{L}$ to $100 \mathrm{ng} / \mu \mathrm{L}$ ), $1 \mathrm{x}$ ThermoPol reaction buffer $\left(10 \mathrm{mM} \mathrm{KCI}, 10 \mathrm{mM}\left(\mathrm{NH}_{4}\right)_{2} \mathrm{SO}_{4}, 20 \mathrm{mM}\right.$ Tris-HCL $(\mathrm{pH}$ $8.8 @ 25^{\circ} \mathrm{C}$ ), 2 mM MgSO $4,0.1 \%$ Triton X-100) (New England BioLabs), 1.5mM $\mathrm{MgCl}_{2}$ (Bioline), $0.4 \mathrm{mg} / \mathrm{mL}$ BSA (Bovine Serum Albumin SIGMA A-2153, fraction V), $250 \mu \mathrm{mol}$ dNTPs, $0.4 \mu \mathrm{M}$ of each primer, 0.75 Unit of Taq DNA polymerase (New England Biolabs) and $\mathrm{ddH}_{2} \mathrm{O}$ to make $25 \mu \mathrm{L}$. 
Products were amplified with the following thermalcycler profile: there was an initial denaturation for 2 minutes at $94^{\circ} \mathrm{C}$, followed by $35-40$ cycles of denaturation for 30 seconds at $94^{\circ} \mathrm{C}$, annealing for 30 seconds at $52^{\circ} \mathrm{C}$, extension for 1 minute at $72^{\circ} \mathrm{C}$, and a final extension step for 5 minutes at $72^{\circ} \mathrm{C}$.

Size of the amplified products for all samples was checked using a 100 base pair (bp) DNA ladder (Roche, Penzberg, Germany) on a 1.5\% agarose gel. The amplified products were cleaned using $1-5 \mu \mathrm{L}$ of exosap (Global Science, Auckland) per $25 \mu \mathrm{L}$ of PCR product. The purified PCR product was then sequenced on an AB13730 Genetic Analyzer by the Allan Wilson Centre Genome Service (Massey University, Palmerston North, New Zealand). Reverse compliments were sequenced to confirm the reads for a number of samples.

\section{Dataset alignment and analysis}

Genetic sequences were aligned using Geneious Pro 3.8.5 (Drummond et al., 2007) with a gap-opening penalty of 12 and a gap-extension penalty of 3 . The final alignment was checked by eye and adjustments made where necessary. Before alignment the sequences were submitted to a BLAST search (http://www.ncbi.nlm.nih.gov/blast/) to confirm that the correct DNA marker had been sequenced. Samples that had been sequenced from forward and reverse directions were combined using Geneious Pro 3.8.5 (Drummond et al., 2007).

\section{Phylogenetic and dating analysis}

MrBayes v3.1.1 (Huelsenbeck, 2001) was used to conduct heuristic searches, implementing the AIC model that was selected by jModeltest (Posada, 2008). The $\operatorname{trnL}-F$ and ITS datasets were analysed separately with four chains and between 2 to 5 million generations, until the standard deviation of split frequencies had fallen below 0.01 , which indicates the runs are stationary. The consensus trees were created excluding $10 \%$ of the trees as burn-in. Ten percent was selected as an appropriate amount to ensure that that the runs had converged on a stationary distribution by considering the standard deviation of split frequencies and exploring the MrBayes output in Tracer (Rambaut and Drummond, 2007). Fifty-percent majority rule 
consensus trees were created for both the $\operatorname{trn} L-F$ and ITS analyses. The congruence of the two datasets was assessed visually by comparing the topology of the chloroplast and ITS Bayesian trees and also by conducting a homogeneity partition test implemented in PAUP* (Swofford, 2002) with 100 replicates, TBR branchswapping and maximum of 1000 trees.

The datasets were combined and then analysed using BEAST v1.4.6 (Drummond and Rambaut, 2007), which was used to estimate divergence times from both gene regions simultaneously. Beast uses a relaxed phylogenetic model, where topology and branch lengths are estimated simultaneously from the data. The topology, including placement of the root node, is not specified a priori (Drummond et al., 2006). The BEAST .xml input file was created with BEAUTi v 1.4.6 (Drummond and Rambaut, 2007), and it was not necessary to estimate parameters independently between data partitions as both the trnL-F and ITS data sets were best estimated by the same model of evolution as determined by jModeltest $(G T R+G)$. A relaxed Bayesian clock with rates for each branch drawn independently from a lognormal distribution was implemented, along with a Yule prior (constant rate of speciation per lineage) (Drummond and Rambaut, 2007). A BEAST run of 10000000 iterations was performed and convergence dates and estimated sample size (ESS) were assessed using Tracer v 1.5 (Drummond and Rambaut, 2007). Means and 95\% higher posterior densities (HPD) of age estimate were obtained from the outputs using Tracer. The 95\% HPD represents the shortest interval that contains $95 \%$ of the sampled values from the posterior (Drummond and Rambaut, 2007). After discarding the first $10 \%$ of samples as burn-in, the samples from the posterior were summarized on the maximum clade credibility tree (the tree that has the maximum sum of posterior probabilities on its internal nodes (Drummond and Rambaut, 2007)) using TreeAnnotator v 1.5 .3 with posterior probability limit set to 0.5 and summarizing mean node heights on. The tree was visualized using FigTree v 1.3.1 (Drummond and Rambaut, 2007).

The relaxed molecular clock (Drummond et al., 2006) was calibrated at two points. First, an internal clade containing Azorina vidalii and Campanula polyclada was given a mean age (tmrca in BEAST) of 6.5 mya with a normal distribution and standard deviation of 0.8 my. These settings gave a prior $95 \%$ confidence interval of 
4.9 mya to 8.1 mya, which correspond to the $90 \%$ highest posterior density interval found by Roquet et al. (2009) for the same node using the penalized likelihood method, and also included their initial maximum age estimate of 8 mya. Roquet et al. (2009) used the endemism of Azorina vidalii (Campanulaceae) on Santa Maria Island in the Azores, which was formed 8 mya (Abdel-Monem et al., 1975), to date the split of $A$. vidalii species from its sister species Campanula dimorphantha, C. polyclada, C. dichotoma, C. molis and C. balfourii. They set a maximum age of 8 mya for this node, and after analyses estimated this node to range from 5.1 to 8 mya (penalized likelihood dating method,) or 3.8 to 7.2 mya (Bayesian relaxed clock method).

The second calibration point was that of the root node (treemodel.RootHeight in BEAST) which corresponds to the split between the platycodonoids and the wahlenbergioids identified by a previous molecular dating study (Roquet et al. 2009). In the present study the prior was set with a normal distribution and a mean at 38.5 mya, the midpoint of Roquet et al's (2009) range from the penalized likelihood dating method, with a range spanning 1.3 standard deviations around this mean. This gave a $95 \%$ confidence interval of 35.95 mya to 41.05 mya, which included in it the estimation of Wikström et al. (2001). Roquet et al. (2009) estimated this node to range from 36 to 41 mya (penalized likelihood dating method), or from 20 to 27 mya (Bayesian relaxed clock method). They had initially set this node with a maximum age of 41 mya based on the dating analysis of Wikström et al. (2001).

\section{Biogeography and character mapping}

Several character traits were mapped onto the nuclear chloroplast and combined Bayesian trees using MacClade v 4.08 (Maddison, 2001). Characters included geographical location (New Zealand, Australia, South Africa, Europe), growth form (herbaceous vs. dwarf-shrub for all areas and radicate vs. rhizomatous for Australasian species), and ploidy levels (Petterson et al., 1995; Smith, 1992). 


\section{Results}

\section{Nuclear (ITS) phylogeny}

The aligned ITS dataset contained 71 samples and was originally 746 characters long. I had difficulties in aligning both the Australasian samples with the South African samples, and all of the Wahlenbergia samples to the outgroup due to high levels of variability in this marker. Homoplasy could thus be a problem in this dataset (Alvarez and Wendel, 2003). Two regions in the alignment, involving bases $50-170$ and $270-510$ were excluded, which resulted in a final alignment of 384 characters. Of these 149 were constant. The model of evolution selected by $\mathrm{j}$ Modeltest was the GTR $+\mathrm{G}$ model. The Bayesian analysis ran for 5 million generations, by which time the standard deviation of split frequencies was $<0.01$.

The $50 \%$ majority consensus tree contains a large number of poorly supported branches (e.g. $<50 \%$ posterior probability (pp) Figure 2.1). Most of the Wahlenbergia samples form a clade (labelled "WAHL" in Figure 2.1, 1.0 pp), with the exception of $W$. hederacea (HEDE_Europe) and $W$. annularis (ANNU_SA_WstC). The $W$. hederacea sample (HEDE_Europe) groups with some Campanula, Jasione and Physoplexus samples, and W. annularis (ANNU_SA_WstC) forms a highly supported clade with the two Lobelia samples. Within the large Wahlenbergia clade there are two main lineages, one containing 23 of the South African species (0.50 pp), and the other the remaining 6 South African species and all of the New Zealand and Australian Wahlenbergia (AUST+1.0 pp). All of the New Zealand species with a radicate growth form a single clade (NZ1, 1.0 $\mathrm{pp}$ ), as do all of the New Zealand species with a rhizomatous habit (NZ2, $1.0 \mathrm{pp}$ ). Interestingly a poorly supported clade of South African species $(0.50 \mathrm{pp})$, containing the Wahlenbergia type species W. capensis (CAPE_SA_WstC), appears to be derived from within the Australasian Wahlenbergia.

\section{Chloroplast (trnL-F) phylogeny}

The aligned trnL-F dataset of 75 individuals was 945 bases long, and 478 nucleotides were conserved across all samples. This dataset was relatively easy to align by eye. 


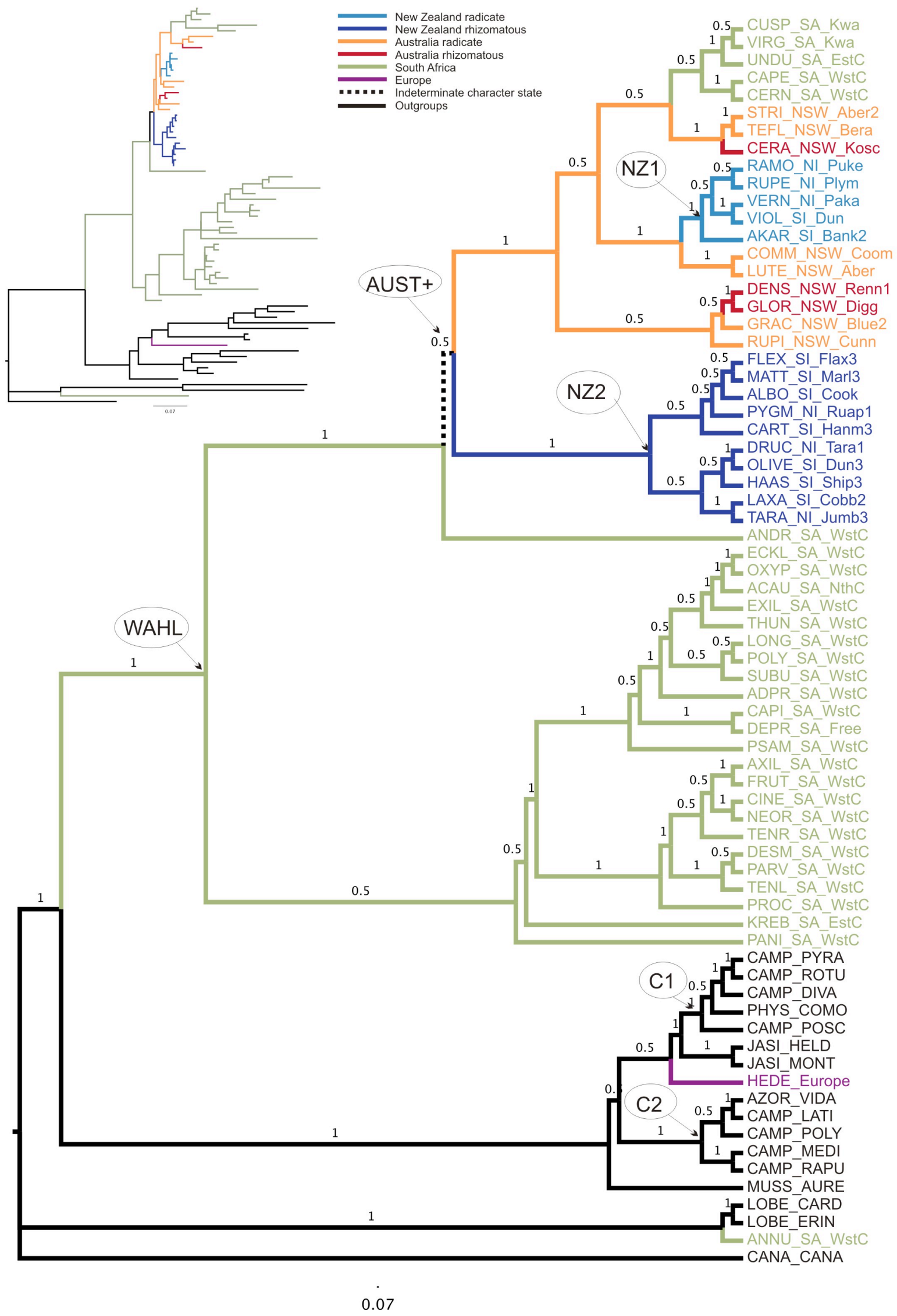

Figure 2.1 Bayesian 50\% majority rule consensus tree of Wahlenbergia (colours) and outgroup (black) based on the ITS dataset. Inset is the phylogram of the same tree. Numbers above branches are posterior probability $(\mathrm{pp})$ values. WAHL $=$ Wahlenbergia clade, AUST $+=($ mostly $)$ Australasian clade, NZ1 = New Zealand species with a radicate growth form NZ2 $=$ New Zealand species with a rhizomatous growth form. C1 and C2 refer to clades in Haberle et al. (2009). See Appendix 1 for an explanation of the tag names and voucher information. 
The model of evolution selected by jModeltest was the GTR $+\mathrm{G}$ model. The Bayesian analysis ran for 2 million generations, by which time the standard deviation of split frequencies was $<0.01$.

All Wahlenbergia samples, excluding $W$. hederacea (HEDE_Europe), form a highly supported clade (WHAL $1.0 \mathrm{pp}$ ) in the 50\% majority rule tree (Figure 2.2).

Wahlenbergia hederacea groups with two Jasione species. Within the Wahlenbergia clade there are three main lineages, the first containing only the South African $W$. krebsii (KREB_SA_EstC), the second comprising 22 of the South African species and the third including the remaining 8 South African species, plus all of the Australasian species sampled. In this way, the Australasian Wahlenbergia, one unidentified South African specimen and the European $W$. lobeliodes (LOBE_Europe) are derived with respect to the South African species in a clade that has 1.0 posterior probability.

\section{Analysis of concatenated dataset}

The homogeneity partition test showed the nuclear vs. chloroplast alignments to be significantly incongruent $(\mathrm{p}=0.01)$. However, this test can be highly inaccurate even when the topologies of trees are congruent (Reeves et al., 2001; Yoder et al., 2001) although in this case visual assessment of the topologies does reveal a level of incongruence. Most of the incongruence appears to be "soft" incongruence, meaning that a particular relationship was resolved in one dataset but not in the other. An example of this is the New Zealand rhizomatous samples all of which form a clade in the ITS tree (clade NZ2 Figure 2.1) but are largely unresolved in the trnL-F tree (clade AUST+ in Figure 2.2). An example of a highly supported "hard" incongruence between the datasets is the placement of a group of five South African species ( $W$. cuspidata, W. virgata, $W$. undulata, $W$. capensis, and $W$. cernua), which are highly supported (1.0 pp) as being basal to the Australasian clade in the trnL-F tree, but are derived within the Australasian species on the ITS tree. Another striking example of a hard incongruence is the placement of $W$. annularis (ANNU_SA_WstC), which formed a highly supported clade with two Lobelia samples in the ITS tree, yet groups with the European $W$. lobeliodes (LOBE_Europe) and the South African $W$. androsacea (ANDR_SA_WstC) in the trnL-F tree. I 

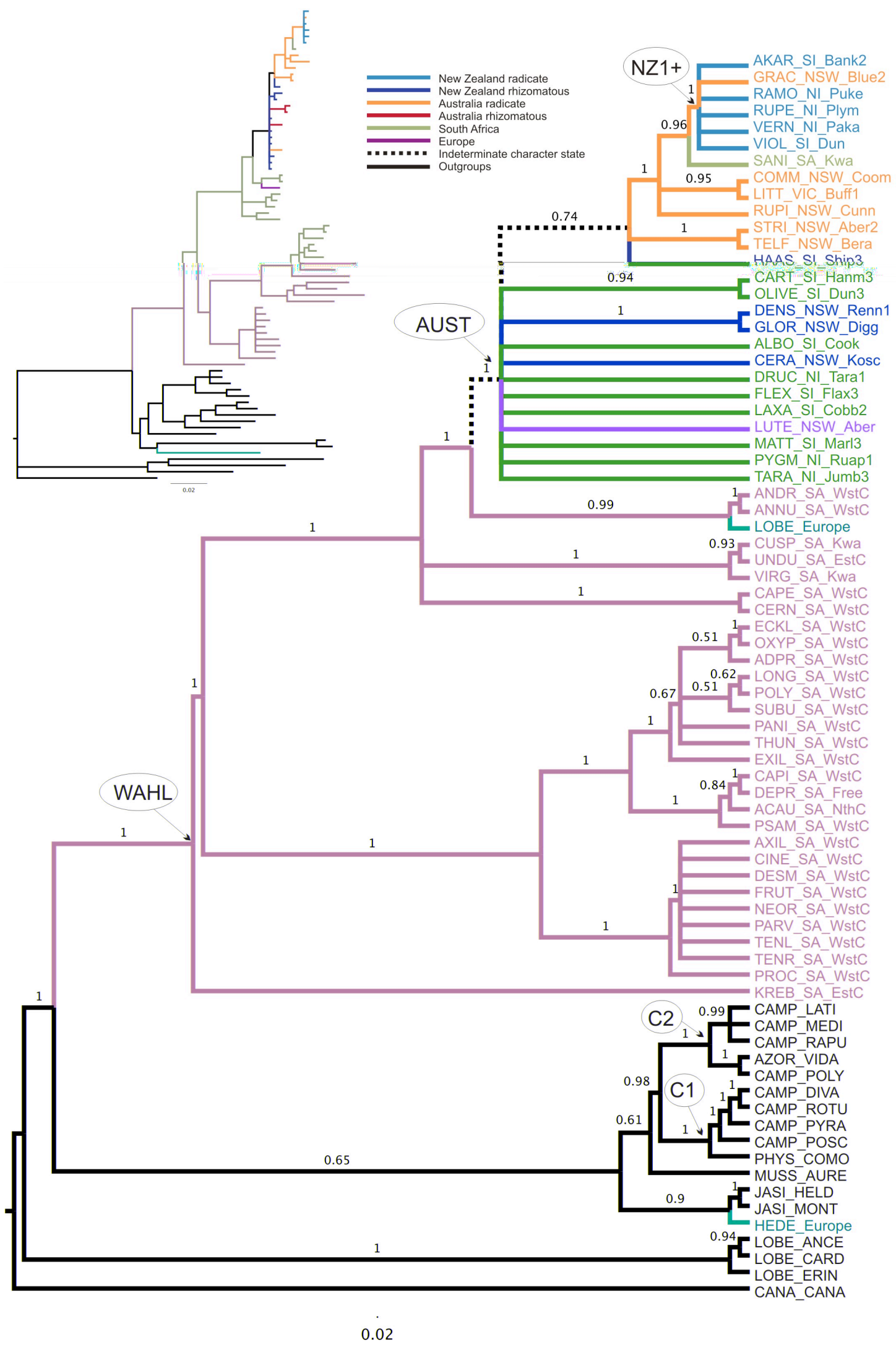

$\mathrm{NZ1+}$
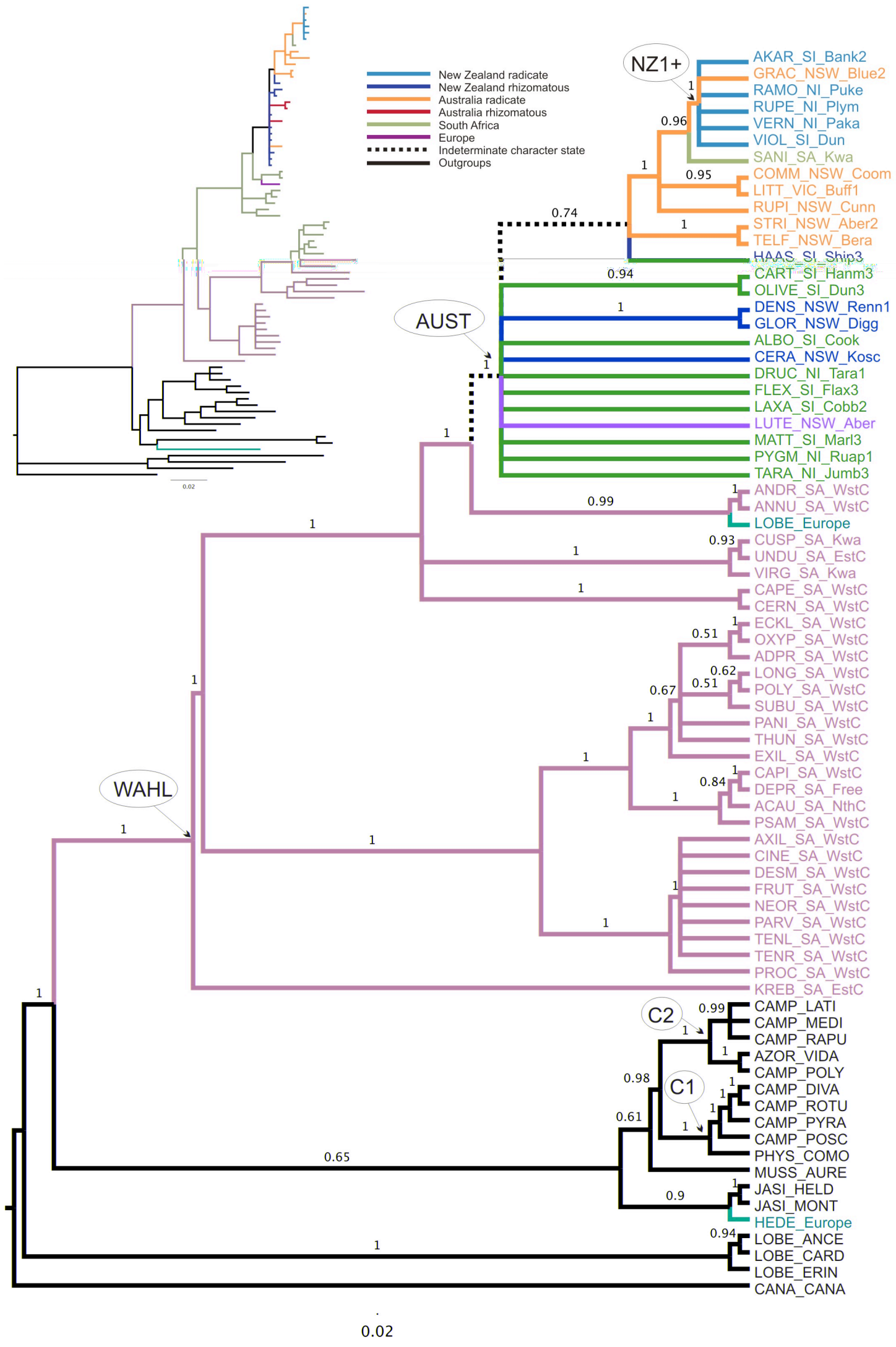
RAMO_NI_Puke

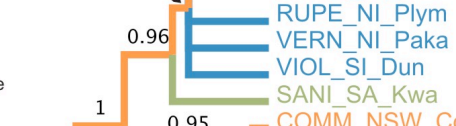
Outgroups

Figure 2.2 Bayesian 50\% majority rule consensus tree of Wahlenbergia (colours) and outgroup (black) based on the trnL-F dataset. Inset is the phylogram of the same tree. Numbers above branches are posterior probability (pp) values. WAHL $=$ Wahlenbergia clade, AUST $=$ Australasian clade, NZ1+ = (mostly) New Zealand species with a radicate growth. New Zealand species with a rhizomatous growth form do not form a clade. $\mathrm{C} 1$ and $\mathrm{C} 2$ refer to clades in Haberle et al. (2009). See Appendix 1 for an explanation of the tag names and voucher information. 
nevertheless proceeded with analysis of the concatenated dataset, with the caveat that the results should be treated with caution due to the incongruence discussed above.

\section{Combined dataset}

The final concatenated dataset was 1329 characters long. The four samples that were missing from the ITS dataset (CAMP_RAPU, LOBE_ANCE, LITT_VIC_Buff1 and LOBE_Europe) were coded as missing data for the ITS characters in the concatenated alignment. The Bayesian phylogeny (Figure 2.3) is generally well resolved at the deeper branches, but there are several polytomies towards the tips of the branches. All Wahlenbergia samples, excluding W. hederacea (HEDE_Europe), form a highly supported clade (clade WAHL $1.0 \mathrm{pp}$ in Figure 2.3). Wahlenbergia hederacea (HEDE_Europe) groups with two Jasione species. Within the Wahlenbergia clade there are three main lineages, the first contains only the South African W. krebsii (KREB_SA_EstC), the second comprises 22 of the South African species and the third includes the remaining 8 South African species, plus all of the Australasian species sampled. Similar to the $\operatorname{trn} L-F$ tree the Australasian Wahlenbergia, one unidentified South African specimen (SANI_SA_Kwa) and the European $W$. lobeliodes (LOBE_Europe) are derived with respect to the South African species in a clade that has 1.0 posterior probability. Within this clade the New Zealand rhizomatous species form a highly supported monophyletic group (NZ2 $1.0 \mathrm{pp}$ ) as do the New Zealand radicate species (NZ1 1.0p p). With respect to the outgroups, the Campanula species sampled fall into two clades, one of which corresponds with the $\mathrm{C} 1$ clade in Haberle et al. (2009), and the other the C2 clade (labelled as such in Figures 2.1 - 2.3). All other outgroup relationships in all three separate and combined are also similar to previous studies.

\section{Molecular dating analysis}

Divergence time estimates are shown on Figure 2.3. The Wahlenbergia clade (excluding $W$. hederacea) is estimated to have diverged 16.19 mya, with a 95\% highest posterior density (HPD) of 10.65 to 21.64 mya (clade WAHL in Figure 2.3). The Australasian Wahlenbergia is estimated to have diverged 3.70 mya, with a $95 \%$ HPD of 2.33 to 5.25 mya (clade AUST Figure 2.3). Within the Australasian clade the dates of divergence of the two New Zealand clades were also estimated. The clade 


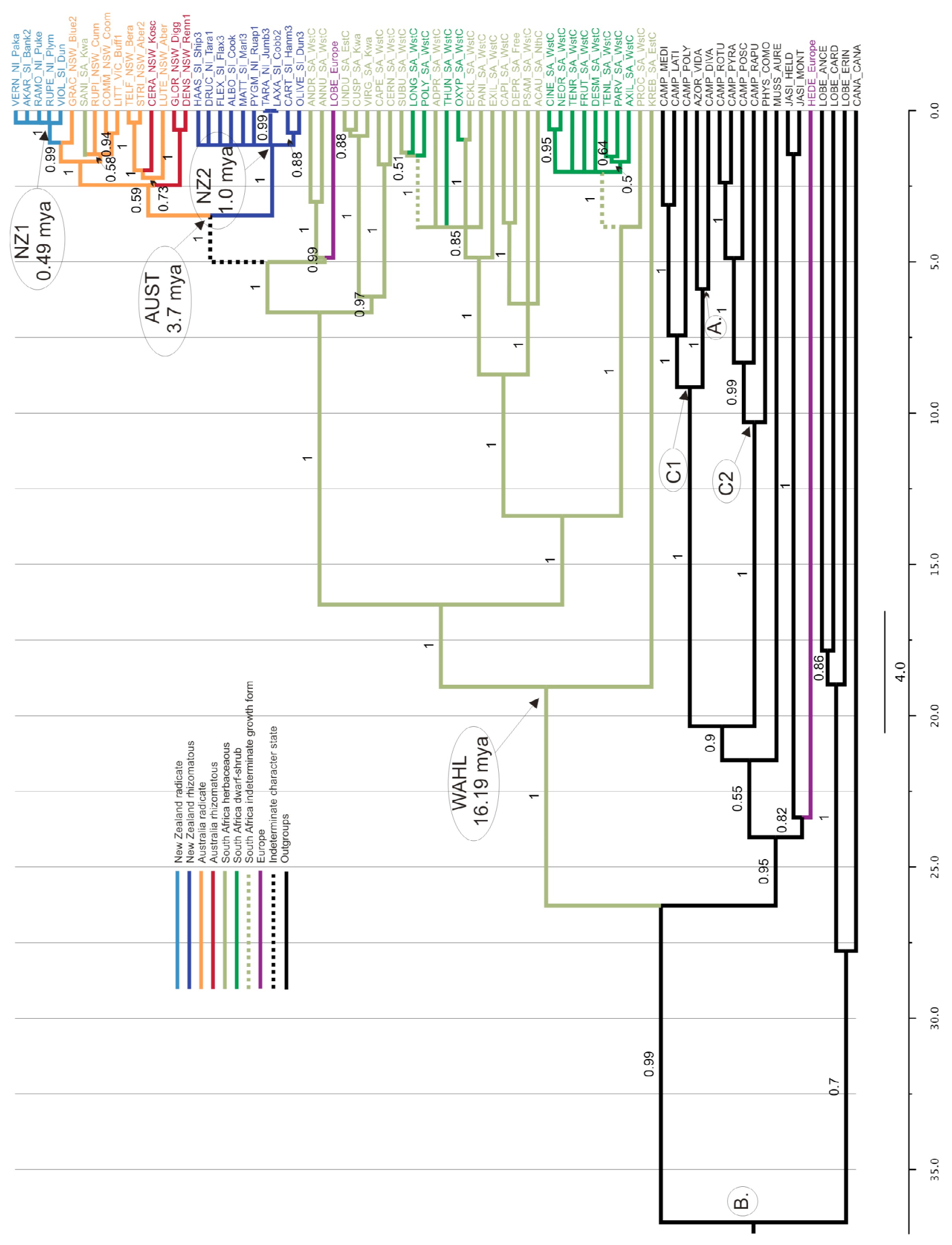

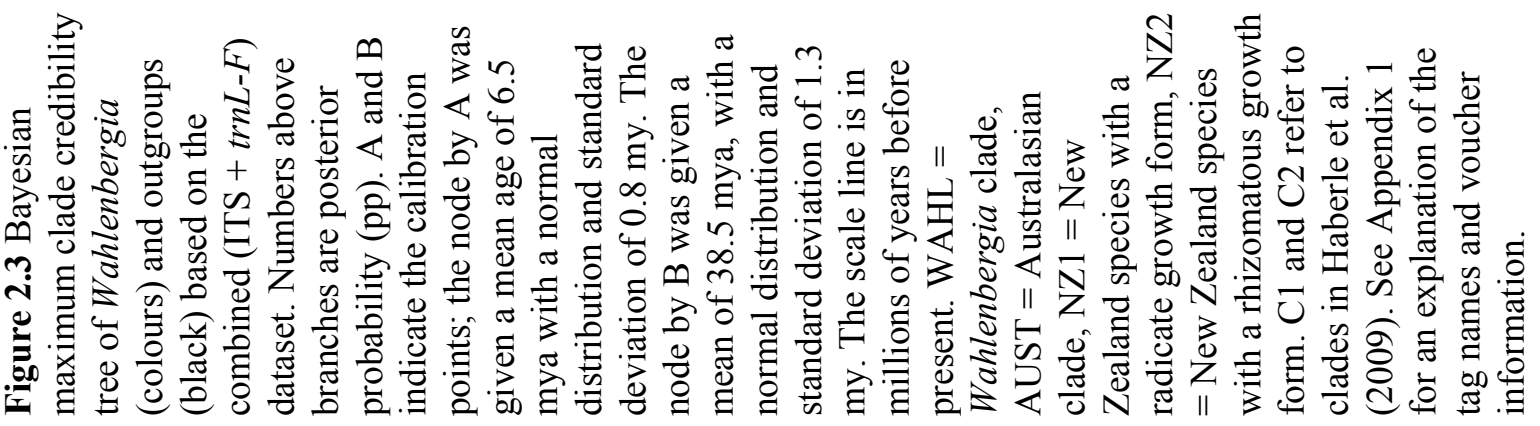


containing all of the New Zealand species with a radicate growth form diverged 0.49 mya, with a 95\% HPD of 0.16 to 0.89 mya (clade NZ1 Figure 2.3 ). The clade containing all of the New Zealand Wahlenbergia with a rhizomatous growth form diverged 1.00 mya, with a 95\% HPD of 0.40 to 1.75 mya (clade NZ2 Figure 2.3).

\section{Biogeography and character mapping}

The results of mapping the geographical location of Wahlenbergia samples onto the individual nuclear, chloroplast and combined trees (displayed on Figures 2.1-2.3) showed the Australasian species to be largely derived with respect to the South African species, which supports a hypothesis of a South African origin for the genus. In the trnL-F and combined trees the Australasian samples form a clade, indicating a single introduction from South Africa to Australasia. Within this Australasian clade the New Zealand and Australian species are not reciprocally monophyletic and instead there are two clades of New Zealand species, (evident in Figure 2.1 and Figure 2.3, but not Figure 2.2), which indicates two separate introductions to, and radiations in, New Zealand.

Plant habit is mapped on to the tree in Figure 2.3. An herbaceous growth form is shown to be the ancestral state for Wahlenbergia as a whole, as well as for the Australasian and South African species, and the dwarf-shrub growth form has evolved multiple times in South Africa. Whether the herbaceous South African Wahlenbergia have a radicate or rhizomatous growth form has not been well recorded, and as it is difficult to determine from herbarium material, it is therefore unknown for most of the species (Chris Cupido pers .comm. 2010). As I was unable to get information relating to this character for the South African species, I only used these terms for the Australian and New Zealand species (Figures 2.1 - 2.3). The New Zealand radicate species form a clade, as do the New Zealand rhizomatous species. The Australian radicate species are paraphyletic however, as two of the Australian rhizomatous species are sister species but the third is nested within a clade of Australian radicate species.

I was unable to satisfactorily map ploidy levels on to the trees, as very few counts have been completed for South African Wahlenbergia, and of the Australian 
Wahlenbergia samples most species have been found to have multiple ploidy levels within the same species. The counts that have been made of South African species have a base of $x=7,8$ or 9 with only one example of polyploidy (Smith, 1992). The Australasian samples that have had their chromosomes counted have all had a base of $x=9$, and the majority are polyploids.

\section{Discussion}

This study is the first to produce a phylogeny of Wahlenbergia including 51 out of ca. 260 species (ca. 20\%). Below, the relationships of the Wahlenbergia samples included in this study are discussed. Biogeographic relationships among Wahlenbergia from different regions (particularly the two main centres of diversity, South Africa and Australasia) and the origin and diversification of Wahlenbergia based on molecular dating techniques are also discussed.

Within the large Wahlenbergia clade (labelled WAHL in Figure 2.3) the South African Wahlenbergia are paraphyletic, with the Australasian Wahlenbergia nested within them in a highly supported clade (1.0 pp). According to the molecular dating analysis, Wahlenbergia evolved ca. 16.19 mya. This suggests the Australasian species, estimated to have arisen ca. 3.7 mya, have all diverged following one longdistance dispersal event from South Africa. This dispersal could have been facilitated by a step-wise progression though Asia, as there are extant species of Asian Wahlenbergia. Nevertheless, long-distance dispersal events from South Africa to Australia are not unknown: for example the Australasian Gnaphaliaea (Asteraceae) taxa were founded by a single trans-Indian Ocean long-distance dispersal event (Bergh and Linder, 2009). Long-distance dispersal over ca. $8000 \mathrm{~km}$ of open ocean seems improbable, but may be facilitated by the westerly winds, which increase in force with greater latitude in the Southern Ocean ('roaring forties', 'furious fifties' and 'shrieking sixties'). Another mode of dispersal is oceanic rafting, which would be made possible by the west wind drift, a large surface current flowing from west to east in the Southern Ocean. The west wind drift would take roughly a year to float objects between South Africa and Australia (Waters and Roy, 2004). Bergh and Linder (2009) discounted the hypothesis that the Gnaphaliaea could have had a 
stepping-stone dispersal through Asia as the Australasian taxa were not nested within a Eurasian clade. The European $W$. lobeliodes included in this study was nested within the South African Wahlenbergia independently of the Australasian Wahlenbergia, and therefore does not implicate a stepping stone dispersal for them, but including Asian Wahlenbergia in future studies will be key to distinguishing between the different biogeographic scenarios.

The phylogenetic and molecular dating results of this study do not support the hypothesis of a Gondwanan history for Wahlenbergia. Rather they suggest the genus probably diverged in Africa during the Miocene, and then dispersed to Australasia where it diverged there relatively recently during the Pleistocene or Pliocene. Further evidence to suggest Wahlenbergia has only been in Australasia for a short while can be found in the fossil pollen record. Wahlenbergia has a distinctive pollen type which is known in New Zealand only from the mid-Pliocene (Waipipian Stage ca. 3.6 mya), and younger sediments in New Zealand (pers comm. Dallas Mildenhall May 2009 and (Muller, 1981)). In Australia there are no published accounts of Wahlenbergia pollen older than quaternary (e.g. (Prebble et al., 2005)), though the absence of evidence cannot be taken as evidence of the absence of Wahlenbergia from Australia before this. A number of recent phylogenetic studies on New Zealand plant groups have found similarly recent dates of arrival and divergence e.g. Pachycladon (Joly et al., 2009), Ourisia (Meudt and Simpson, 2006), Ranunculus (Lockhart et al., 2001) and Sophora (Hurr et al., 1999).

In this study relationships within the South African Wahlenbergia are often resolved with high support. Chris Cupido will discuss relationships within the South African Wahlenbergia further when this chapter is readied for publication as they are his data and I am unknowledgeable about the South African samples.

Relationships within the highly supported Australasian clade (AUST) are poorly resolved, but it is clear that the Australian and New Zealand species are not reciprocally monophyletic. The inclusion of one unidentified South African sample in the Australasian clade is very interesting, and could possibly represent an Australasian species recently introduced by anthropogenic means to South Africa, though attempts to identify it using keys for the Australasian species have been 
unsuccessful (Chris Cupido pers comm. 2010). Alternatively, it could represent a natural long-distance dispersal event from Australia to South Africa, which has been documented in other plant groups e.g. Patersonia and Geosiris (Iridaceae) (Goldblatt et al., 2002). In a literature survey by Bergh and Linder (2008) dispersals in the easterly direction across the Indian Ocean have occurred eight times in the last 55 million years, though they ventured no possible mechanisms for dispersals in this direction. Additionally the South African species nested within the Australasian clade in the ITS tree could represent an additional dispersal from Australia to South Africa, but this requires further investigation.

New Zealand specimens are grouped into two highly supported clades within the Australasian clade (NZ1 and NZ2 in Figure 2.3). All of the samples in NZ1 have the radicate growth from, whereas those in NZ2 have the rhizomatous growth form. The position of NZ2 is unresolved, but NZ1 is nested within a clade containing four Australian species and the unidentified South African species. This topology probably suggests two long-distance dispersal events from Australia resulting in clades NZ1 and NZ2 (even though the placement of NZ2 is unresolved). Long distance dispersal from Australia to New Zealand is a common occurrence (e.g. Pittosporum (Chandler et al., 2007; Gemmill et al., 2002), Elaeocarpus (Crayn et al., 2006), Charmichaelia (Wagstaff et al., 1999) and Polystichum (Perrie et al., 2003)). The mechanisms for dispersal across the $2000 \mathrm{~km}$ of Tasman Sea that separates New Zealand and Australia remain unclear (Winkworth et al., 2002b) although wind dispersal has been advocated for plants with small, lightweight seeds. The seeds of Wahlenbergia are lightweight, but without any obvious modifications for winddispersal (Petterson, 1997b). In other cases biotic vectors such as migratory birds, or sea birds have been implicated (Winkworth et al., 2002b).

An herbaceous growth form was shown to be the ancestral state for Wahlenbergia as a whole, as well as for the Australasian and South African species. Unfortunately, whether species of herbaceous South African Wahlenbergia have a rhizomatous or radicate growth form is largely unrecorded (Chris Cupido pers. comm. 2010). It would be of great interest to determine what growth form the ancestor of the Australasian species that dispersed from South Africa had. 
Chromosome numbers were unable to be mapped, as few counts have been made on African species, and most of the Australian species with the radicate growth form have more than one ploidy race. Nevertheless, there does appear to be a pattern of increasing ploidy levels as the genera has evolved, as South African species are mostly diploids, and with a base of $x=7,8$ or 9 , whereas the Australasian species all have a base of $x=9$ and all are polyploids. It appears likely that the ancestor of the Australian species that dispersed from South Africa was of the $\mathrm{x}=9$ group, as Smith (1992) refers to a South African "W. undulata group", which consists of 6 species including $W$. undulata and $W$. virgata (included in this study). The group is characterized by a base chromosome number of $\mathrm{x}=9$, a distinct corolla-tube and usually stylar glands. Smith (1992) hypothesised that the general affinities of the native Australian species were with the $W$. undulata group in South Africa, and the placement of $W$. undulata and $W$. virgata with regards to the Australasian species in this study (Figure 2.3) offers some support for that hypothesis. However, further chromosome counts of both the South African and Australian species are necessary to explore this issue further.

Wahlenbergia is confirmed to be non-monophyletic by this study, with $W$. hederacea from Europe grouping instead to the two sampled species of the outgroup Jasione with high support (Figure 2.3). All remaining Wahlenbergia included in this study form a highly supported clade (1.0 pp in trnL-F and combined datasets). Note however, that my sampling includes only species from South Africa, Australia, New Zealand and southern Europe. Unfortunately, no Wahlenbergia samples from Asia, South America or any Pacific islands were able to be included in this study. To fully assess the monophyly of Wahlenbergia the next important step will be to include Wahlenbergia from these unsampled locations, as well as a selection of other closely related walenbergioid species (e.g. Microcodon glomeratum and Theilera guthriei included in Haberle et al. (2009)) since previous studies have shown Wahlenbergia to be polyphyletic even within the wahlenbergioid clade (Haberle et al. 2009, Roquet et al. 2009).

The relationship of $W$. hederacea with the Jasione species in the campanuloid clade has been recovered in all studies that have included these species (e.g. (Eddie et al., 2003), (Haberle et al., 2009), and (Cellinese et al., 2009)). Their relationship to the 
rest of Campanulaceae was unresolved in Eddie et al.'s (2003) ITS phylogeny, but in all subsequent studies (e.g. chloroplast gene order (Cosner et al., 2004), ITS \& trnL$F$ (Roquet et al., 2009) and $a t p B$, matK and $r b c L$ (Haberle et al., 2009)) they have been resolved as part of the campanuloids. Based on their apical dehiscence $W$. hederaceae and Jasione species were included in subtribe Wahlenbergeae by De Candolle (1830). However, as noted by Haberle et al. (2009) Jasione in particular also share characters with the campanuloids such as Campanula jacquinii with which they share characters like flowers in terminal congested heads, subtended by a bract. These results taken together strongly suggest that Wahlenbergia hederacea is currently in the incorrect genus and should be considered a campanuloid rather than a wahlenbergioid species. Further studies to find its sister species would be necessary before any taxonomic changes could be undertaken.

The ITS dataset also had one South Africa Wahlenbergia (ANNU_SA_WstC) grouping with two Lobelia samples rather than with the other South African Wahlenbergia as it did in the trnL-F and combined datasets. While this could represent the true relationship, potentially indicating hybridisation with Lobelia, it could also be an artefact of the difficulties in aligning the ITS dataset. Due to the high level of sequence divergence, homoplasy could well have been introduced into this dataset (Alvarez and Wendel, 2003).

Other subfamilial relationships found in this study, including two clades of largely Campanula species, match subfamilial relationships found in Roquet et al. (2009), Haberle et al. (2009) and Eddie et al (2003) but with more limited sampling in this case. The clade labeled C1 in Figure 2.3 matches Haberle et al.'s (2009) C1 clade, and Roquet et al.'s (2009) Campanula sensu strictu. The clade labeled C2 in Figure 2.3 matches Haberle et al.'s C2 clade, and Roquet et al.'s Rapunculus clade.

\section{Summary and Conclusions}

In this study, the genus Wahlenbergia is confirmed to be polyphyletic, although the majority of species form a clade including the type species $W$. capensis. Further sampling is required to confirm whether this monophyly extends to Asian and Pacific 
Wahlenbergia, and whether it is maintained when other wahlenbergioid species are included in the dataset. The genus originally evolved in South Africa and began diversifying about 16.2 mya before dispersing to Australasia and radiating about 3.7 mya. It is unclear whether this dispersal event included a stepping stone path through Asia, as unfortunately no Asian Wahlenbergia were included in this study. Two introductions to New Zealand appear likely, leading to two radiations: one of radicate and one of rhizomatous species. The New Zealand rhizomatous species arrived and diverged about 1 mya, and the radicate species about 0.5 mya. An herbaceous habit was shown to be the ancestral state for the genus as a whole. Although chromosome numbers were unable to be mapped there does appear to be a pattern of increasing ploidy levels as the genus has evolved. The recent evolution of the Wahlenbergia genus as a whole and the recent introductions to New Zealand refute the Gondwanan vicariance hypothesis for the origins of the New Zealand Wahlenbergia and instead provide overwhelming evidence for arrival via long distance dispersal. 


\title{
Chapter Three: Phylogenetic analysis of three DNA sequencing markers to explore relationships within Australasian Wahlenbergia
}

\begin{abstract}
A phylogeny of the Australasian Wahlenbergia of 105 individuals, representing 64\% of the species present in Australasia, was reconstructed based on the nuclear ribosomal ITS marker and the chloroplast $\operatorname{trn} L-F$ and $\operatorname{trn} K$ markers following a primer trial of 22 nuclear, chloroplast and mitochondrial markers. Low levels of genetic variation among individuals from Australia and New Zealand was revealed with all markers, and the phylogenies were poorly resolved as a result, a finding that is in line with other published molecular phylogenetic studies on other New Zealand groups. Conflict between the nrITS and chloroplast markers coupled with this low genetic diversity is probably due to rapid and recent evolution during a period of geological and climatic change, perhaps coupled with incomplete lineage sorting and/or hybridisation. Despite the poor resolution, several morphological species were recovered as monophyletic, notably the morphologically distinctive New Zealand $W$. cartilaginea, $W$. matthewsii and $W$. congesta subsp. congesta. The lowland radicate $W$. gracilis complex may all belong to the same morphologically variable species. In contrast, the other New Zealand radicate species, $W$. vernicosa, is probably a separately-evolving lineage, and is not conspecific with the $W$. gracilis complex or the Australian $W$. littoricola as previously hypothesized. Two recently described Australian species ( $W$. rupicola and $W$. telfordii) formed monophyletic groups and thus the species rank was supported.
\end{abstract}




\section{Introduction}

The genus Wahlenbergia is one of the largest genera within the Campanulaceae, comprising upwards of 260 species (Haberle et al., 2009). It is distributed mainly in the southern hemisphere, although also extends north of the equator into western Europe and eastern Asia (Lammers, 1996). The centre of diversity is South Africa (ca. 81\% of Wahlenbergia species), with a secondary centre in Australasia (ca. 13\%) (Petterson, 1997b; Smith, 1992). The genus is also indigenous to New Caledonia, New Guinea, Malaysia, Japan, Taiwan, China, India and some of the small volcanic Pacific islands, e.g., Juan Fernández and Tonga (Petterson, 1997b). The Australasian centre of diversity is the focus of this chapter. The previous chapter in this thesis found the Australasian Wahlenbergia to be monophyletic (including one unidentified South African specimen), derived with respect to the remaining South African species and with two introductions hypothesised from Australia to New Zealand. The focus of this chapter will be on testing the current morphology-based taxonomy of New Zealand Wahlenbergia using modern molecular systematic techniques. The present study will be the first dedicated phylogenetic study of the Australasian Wahlenbergia and builds on morphological revisions of New Zealand (Petterson 1997b) and Australian (Smith 1992) species.

The current Wahlenbergia taxonomy in New Zealand is mostly based on a recent morphological revision by Judith Petterson (1997b). Petterson's revision represents the culmination of a lifetime of study of a very difficult genus (de Lange, 2005) beginning with her Masters thesis on Wahlenbergia (Petterson, 1953). In her 1997 revision, she provided taxonomic treatment for ten indigenous species, nine of them endemic and one also found on the Kermadecs and possibly Tonga. Of these, three species are further divided at subspecies rank, providing an addition seven endemic subspecies (Table 1.1). Petterson primarily used morphological and ecological characters to distinguish species, and did not attempt any phylogenetic assessment or analysis. A base chromosome number of $\mathrm{x}=9$ is inferred for the New Zealand species, which can be separated into four convenient groups based on growth form and chromosome number: temperate radicate $(2 n=72,4$ octaploid species), subtropical radicate $(2 n=54,1$ hexaploid species), suffructose rhizomatous ( $2 n=36,1$ tetraploid species) and creeping rhizomatous $(2 n=36,4$ tetraploid species) (Petterson, 
1997b; Petterson et al., 1995), growth forms are illustrated in Figure 1.1. There are no diploids $(2 n=18)$ found in New Zealand, suggesting our species might have been derived from polyploidy ancestors.

The current Australian Wahlenbergia revision (Smith, 1992) included 26 species, 25 native and one introduced. This treatment is generally well accepted, though some state-specific Australian flora include slightly different treatments, e.g. the Flora of Victoria considers $W$. littoricola as a synonym of $W$. graniticola (Walsh, 1999). The Australian species are all herbaceous annuals or perennials, and either have a rhizomatous or tufted growth form (Smith, 1992). The radicate growth form of Petterson (1997b) is equivalent to the tufted growth form of Smith (1992). Several chromosome counts of Australian Wahlenbergia have been undertaken (summarized in Smith (1992)), and a base chromosome number of $x=9$ is evident. Plants with the rhizomatous growth form are all diploids $(2 n=2 \mathrm{x}=18)$. Plants with the tufted growth form range from diploids $(2 n=18)$ to octoploids $(2 n=72)$, with most species comprising at least two chromosome races (Smith, 1992).

\section{Research aims}

Developments in molecular techniques such as DNA sequencing have provided taxonomists with a wealth of new characters to help make taxonomic decisions, and programs for analyzing these characters (and morphological characters) by creating phylogenies are now well established in the field. Phylogenetic trees provide the framework for the most meaningful evolutionary comparisons (Soltis and Soltis, 2000). Phylogenetic studies of New Zealand plants using DNA sequences as characters have been useful in aiding taxonomic decisions in a whole range of taxa e.g. Veronica (Garnock-Jones et al., 2007) and Pachycladon (Heenan et al., 2002).

In this chapter, I present the first molecular phylogeny of the Australasian Wahlenbergia, with the primary aim of testing the current New Zealand Wahlenbergia taxonomy, although questions regarding the Australian species will also be addressed where sampling permits. Appropriate markers for DNA sequencing have been selected from a trial of organellar DNA (chloroplast and 
mitochondrial) and nuclear DNA (both low-copy and ribosomal). Specific questions include:

1. species boundaries within the New Zealand $W$. gracilis complex,

2. the status of $W$. littoricola subsp. vernicosa,

3. the status of subspecies of the New Zealand rhizomatous species e.g. those of $W$. albomarginata and $W$. pygmaea and

4. monophyly of the newly described Australian species $W$. rupicola and $W$. telfordii.

These specific questions are further introduced below, along with the species and species complexes they relate to.

\section{Taxonomic introduction}

\section{New Zealand radicate species}

All of the New Zealand radicate species inhabit lowland habitats. Morphologically they appear very similar - especially when dried and pressed - and precise floral measurements are often necessary to distinguish them. The five radicate species have a lively taxonomic history (Petterson, 1997a) and were originally considered to be one species - Campanula gracilis (Forster, 1786). Four of these species are temperate and one, $W$. vernicosa, is subtropical.

The four endemic temperate radicate species ( $W$. violacea, $W$. akaroa, $W$. ramosa and $W$. rupestris) share a chromosome number of $2 n=72$. They are hard to distinguish, and hybrids $(W$. violacea $\times W$. rupestris, $W$. violacea $\times W$. akaroa and $W$. rupestris $\times W$. akaroa) have been reported (Petterson, 1997b). Webb and Simpson (2001) referred to the four temperate radicate species as a single species: Wahlenbergia gracilis (hereafter referred to as the $W$. gracilis complex). They found no way to distinguish between the seeds of any of the five radicate species, but accepted that $W$. vernicosa should be considered a separate species due to its different chromosome count. The radicate species are characterised mainly based on floral characters. $W$. violacea and $W$. akaroa have blue/purple flowers, $W$. akaroa is restricted to Banks Peninsula and has flowers approximately twice as large as $W$. 
violacea. $W$. ramosa and $W$. rupestris have white or pale blue flowers, and are distinguished by $W$. rupestris having non-overlapping elliptic shaped petals, compared to W. ramosa having ovate shaped petals that overlap (Petterson, 1997b). The taxonomy is further confused by the enigmatic $W$. colensoi, which was treated as a starved form of $W$. ramosa, $W$. rupestris or $W$. violacea in Petterson (1997b), but has been found growing in sympatry with more typical samples of these species (JMP, pers. obs. based on notes on herbarium specimens). Whether these entities should be considered varieties, subspecies or species, and how many should be recognised, are unresolved questions. I address species boundaries and ranks in the $W$. gracilis complex by using DNA sequence data to test whether clades based on DNA sequences match the taxonomy based on morphological characters.

The indigenous tropical radicate species, $W$. vernicosa, has a different chromosome count of $2 n=54$ which is unique in New Zealand. Subsequent to Petterson's revision, de Lange and Cameron (1999) reduced $W$. vernicosa to a subspecies of the otherwise Australian $W$. littoricola on the basis that the only morphological character to distinguish $W$. vernicosa from $W$. littoricola is its glossy leaf surface. Petterson (2005) contested this reduction to subspecies rank, but without providing any further evidence for her rejection of the change this matter deserves further investigation. Here, I use DNA sequence data to examine the origins of this hexaploid species (in the absence of a diploid New Zealand species as a putative parent) and to test its proposed conspecificity with Australian W. littoricola.

\section{New Zealand rhizomatous species}

All of the rhizomatous species in New Zealand share the same chromosome count of $2 n=36$. Only one species has the suffructose rhizomatous growth form and four have creeping rhizomatous habit.

The suffructose $W$. matthewsii is easy to identify as a species because of its unique growth form, along with its limestone habitat. Putative hybrids between $W$. matthewsii and W. albomarginata from Castle Hill and Prebble Hill are represented by herbarium specimens at the Allan Herbarium (CHR). These have the creeping rhizomatous growth form characteristic of $W$. albomarginata, yet linear leaves 
characteristic of $W$. matthewsii. Some of these samples (e.g. CHR 76373) were previously identified as $W$. brockiei (Petterson, 1997b). Wahlenbergia brockiei was not recognized by Judith Petterson as she considered the type to be a dry habitat form of $W$. albomarginata (Petterson, 1997b) based on growth observations of $W$. brockiei and $W$. albomarginata in a common garden. Unfortunately, $W$. brockiei was not able to be sampled for the present study.

There are four species with the creeping rhizomatous growth form: Wahlenbergia. albomarginata, $W$. pygmaea, $W$. cartilaginea and $W$. congesta. $W$. congesta is a relatively distinctive species, being the only consistently coastal rhizomatous Wahlenbergia in New Zealand. It inhabits the west coast of the South Island. Petterson (1997) recognised two subspecies, W. congesta subsp. haastii is characterised by smaller capsules and flowers than $W$. congesta subsp. congesta. The most distinctive Wahlenbergia in New Zealand is $W$. cartilaginea, which is restricted to scree slopes in the northwestern South Island and has very thick glaucous cartilaginous leaves and calyx lobes. A number of New Zealand genera each contain just one or a few scree-adapted species (e.g., Stellaria roughii, Lobelia roughii, Notothlaspi rosulatum, Ranunculus haastii and $R$. acraeus), suggesting the scree habitat might be a relatively recent product of greywacke mountain uplift and erosion cycles that include glaciation and interglacial aridity and wind. W. cartilaginea is more locally distributed than many of the others, which might indicate a very recent origin.

Wahlenbergia albomarginata (sensu Judith Petterson) is restricted to the South Island, primarily in alpine habitats. It has five subspecies, which are distinguished by leaf characters combined with geographic and geological (e.g. edaphic) separation. Several of the five subspecies are difficult to identify in practice (e.g. $W$. albomarginata subsp. albomarginata (dry areas of eastern South Island, Central Otago, Fiordland) vs. W. albomarginata subsp. laxa (inland and wetter areas of South Island) and W. albomarginata subsp. decora (higher altitude areas)), as their leaf morphology can be intermediate. W. albomarginata subsp. flexilis is easy to distinguish as it is restricted to eastern Marlborough limestone, though it would be difficult to identify based on morphological characters alone (JMP pers. obs.). The remaining subspecies, $W$. albomarginata subsp. olivina, is the only subspecies 
consistently able to be identified using the key in Petterson (1997b) as it differs both in habitat requirements (it grows on ultramafic serpentine rock outcrops) and in stable morphological characters (it has consistently thicker leaf margins).

$W$. pygmaea sensu Judith Petterson effectively replaces $W$. albomarginata in the North Island as the common alpine species. It is generally a much smaller plant as its name suggests, and in the past small South Island $W$. albomarginata plants have been identified as $W$. pygmaea (JMP pers. obs. of herbarium specimen labels at WELT and NSW). Other than size of plants the main character that separates $W$. albomarginata from $W$. pygmaea is the shape of the flowers. W. albomarginata has a 'narrow-campanulate-rotate corolla, with tube distinctly longer than broad' whereas in $W$. pygmaea the corolla is 'broadly campanulate, with tube as wide as, or wider than long' (Petterson, 1997b). There are three subspecies of W. pygmaea currently recognised, defined primarily by geography, but also apparently by floral and foliar differences (Petterson, 1997b). Wahlenbergia pygmaea subsp. pygmaea is found growing on the central plateau of the North Island and south into the Ruahine ranges. It has leaves that are entire or with 2-4 teeth on each side. Wahlenbergia pygmaea subsp. tararua is restricted to the Tararua ranges, and only one population is known from there now. Its leaves are serrate, with 4-7 teeth on each side. Doubts have been expressed as to whether there is sufficient evidence to consider this a separate subspecies and recently it was excluded from the naturally uncommon species list, on the basis that it was taxonomically indistinct (de Lange et al., 2009). Wahlenbergia pygmaea subsp. drucei is restricted to Mt Taranaki. It subtly differs morphologically by having dentate leaves with 4-7 teeth on each side and broader capsules than W. pygmaea subsp. pygmaea.

Despite the floral differences described above it is not possible to distinguish between $W$. pygmaea and $W$. albomarginata on the basis of their seeds. However, seeds may not be a useful character in this group, because seeds of the otherwise morphologically distinct South Island west coast endemic $W$. congesta cannot be distinguished from those of these two species either (Webb and Simpson, 2001). In this thesis I use molecular characters to examine the monophyly and relationships of these two species and their intraspecific taxa as an independent test of their status. 


\section{Australian species}

There were 25 native species included in Smith's 1992 revision, 19 of which have a radicate ("tuffed") growth form, and six have a rhizomatous growth form. The radicate species can be further split into three groups based on morphological and cytological evidence (Smith, 1992). The W. scopulicola group consists of rockcrevice growing species, $W$. scopulicola, $W$. glabra and $W$. islensis. The $W$. gracilenta group consists of all of the species with an annual life cycle, $W$. gracilenta, $W$. pressii, $W$. victoriensis and $W$. caryophylloides. The $W$. communis group is the largest group, consisting of twelve species. These share the same growth form and life cycle as the New Zealand radicate species and are the most in need of revision (Smith, 1992). The members include W. stricta, W. luteola, $W$. communis, $W$. queenslandica and three subgroups of closely related species. The $W$. graniticola subgroup consists of $W$. graniticola, $W$. aridicola and $W$. littoricola. The $W$. planiflora subgroup consists of $W$. planiflora, $W$. fluminalis and $W$. multicaulis and the $W$. gracilis subgroup consists of $W$. gracilis and $W$. tumidifructa.

The rhizomatous species (termed the $W$. gloriosa group in Smith (1992)) include $W$. ceracea, $W$. gloriosa, $W$. densifolia, $W$. gymnoclada, $W$. saxicola and $W$. insulaehowei. Smith (1992) conjectured the New Zealand rhizomatous species were related to the $W$. gloriosa group, and suggested the group were most likely to have spread from Australia to New Zealand, rather than the other way around, based on the increased ploidy levels of the New Zealand specimens.

Subsequent to Smith's (1992) revision two new Australian radicate species have been described, W. rupicola and W. telfordii (Plunkett et al., 2009). A dendrogram based on phonetic analysis of morphological characters grouped $W$. telfordii with individuals of $W$. glabra, W. ceracea and W. stricta. Wahlenbergia rupicola grouped most closed with $W$. luteola, though had similar seed morphology to $W$. ceracea, and a superficial morphological resemblance to $W$. stricta. Here I use molecular characters to examine the monophyly and relationships of these two new species as an independent test of their status. 


\section{Methods}

\section{Taxon sampling}

Location of samples, collection details, voucher information and GenBank accession numbers are presented in Appendix 1. A total of 105 individuals are included in this study, including 94 Australasian Wahlenbergia individuals (representing 29 of the 45 species and subspecies), two European Wahlenbergia individuals and nine outgroup individuals. Fifteen of the 17 species and subspecies of New Zealand Wahlenbergia (Petterson, 1997b) are represented by 54 individuals in this study. The only New Zealand Wahlenbergia taxa not included in this study are $W$. congesta subsp. congesta and $W$. albomarginata subsp. decora. Six species and two subspecies are represented by more than one sampled population e.g. W. violacea, W. littoricola subsp. vernicosa and $W$. albomarginata subsp. albomarginata. This was not practical for species with more restricted distributions, and in some cases all samples of a particular species/subspecies are members of the same population e.g. W. akaroa and $W$. albomarginata subsp. flexilis. Ten populations are represented by more than one plant.

The Australian Wahlenbergia are represented in this study by 40 samples comprising between 1 and 6 individuals of 14 of the 28 species present in Australia as classified by Smith (1992) and (Plunkett et al., 2009). Not all Australian species were able to be included in this study due to collection constraints - most species present in New South Wales were collected on one trip to this state, and additional samples were extracted from herbarium specimens or sent by other researchers. Sequences of European Wahlenbergia and some outgroups were downloaded from GenBank (http://www.ncbi.nlm.nih.gov/) and were selected to represent closely related genera following Haberle et al. (2009).

\section{DNA isolation, amplification and sequencing}

Total DNA was extracted from silica-dried leaves after manual disruption of dried tissue with a mortar and pestle using a (CTAB) method modified from Doyle \& Doyle (1990) and explained in detail in Chapter Two of this thesis. 
Leaves from older herbarium specimens from the herbarium at the museum of New Zealand Te Papa Tongawera (WELT) collected between 1994-1997 were removed with permission and extracted in a dedicated ancient DNA (aDNA) lab at Massey University, Palmerston North. These samples were extracted using a Qiagen DNeasy plant mini extraction kit following the manufacturers instructions. Half of the eluted aDNA was then returned to the modern lab at Victoria University for amplification and sequencing, with half stored in the aDNA lab for future use.

Twenty chloroplast, mitochondrial and nuclear DNA sequencing markers were trialled. A list of the primers trialled can be found in Table 3.1. Selection criteria for successful primers included those that amplified cleanly (single bands) and universally across a selection of different Wahlenbergia. The primers were trialled on the Australian radicate W. communis (COMM_NSW_Quea), the New Zealand temperate radicate $W$. violacea (VIOL_SI_Dun) and two New Zealand creeping rhizomatous subspecies: W. albomarginata subsp. laxa (LAXA_SI_Cobb1) and $W$. albomarginata subsp.albomarginata (ALBO_SI_Garv). PCR amplification and sequencing of the trial markers followed protocols outlined in the references in Table 3.1. Protocols for the markers eventually chosen for this study (the nuclear ribosomal DNA marker ITS, and the chloroplast DNA regions $\operatorname{trn} L-F$ and $\operatorname{trn} K$ ) are outlined below.

The oligonucleotide primers for the three loci ( $\operatorname{trn} L-F, \operatorname{trn} K$ and ITS) are listed in Table 3.1. PCR amplification was performed in a Biometra $\mathrm{T}$ gradient machine (Whatman, Germany) in a final volume of $25 \mu \mathrm{L}$. Each $25 \mu \mathrm{L}$ volume contained $2 \mu \mathrm{L}$ of DNA template (nanodrop concentrations ranged from $20 \mathrm{ng} / \mu \mathrm{L}$ to $100 \mathrm{ng} / \mu \mathrm{L}$ ), $1 \mathrm{x}$ ThermoPol reaction buffer $\left(10 \mathrm{mM} \mathrm{KCI}, 10 \mathrm{mM}\left(\mathrm{NH}_{4}\right)_{2} \mathrm{SO}_{4}, 20 \mathrm{mM}\right.$ Tris-HCL $(\mathrm{pH}$ $8.8 @ 25^{\circ} \mathrm{C}$ ), 2 mM MgSO $4,0.1 \%$ Triton X-100) (New England BioLabs), 1.5mM $\mathrm{MgCl}_{2}$ (Bioline), $0.4 \mathrm{mg} / \mathrm{mL}$ BSA (Bovine Serum Albumin SIGMA A-2153, fraction $\mathrm{V}), 250 \mu \mathrm{mol}$ dNTPs, $0.4 \mu \mathrm{M}$ of each primer, 0.75 Unit of Taq DNA polymerase (New England Biolabs) and $\mathrm{ddH}_{2} \mathrm{O}$ to make $25 \mu \mathrm{L}$. In some instances $4 \%$ v/v of Betaine (N,N,N-trimethylglycine) and/or 4\% v/v DMSO (Dimethyl sulfoxide) were added to the master mix to optimise the PCR conditions when amplifying ITS 
fragments. The volume of $\mathrm{ddH}_{2} \mathrm{O}$ was altered to maintain a reaction volume of $25 \mu \mathrm{l}$ in the event of such additions.

Products were amplified with the following thermalcycler profiles. For the $\operatorname{trn} L-F$ region and the ITS region there was an initial denaturation for 2 minutes at $94^{\circ} \mathrm{C}$, followed by $35-40$ cycles of denaturation for 30 seconds at $94^{\circ} \mathrm{C}$, annealing for 30 seconds at $52^{\circ} \mathrm{C}$, extension for 1 minute at $72^{\circ} \mathrm{C}$, and a final extension step for 5 minutes at $72^{\circ} \mathrm{C}$. The other chloroplast region, $\operatorname{trn} K$, was amplified using the following profile: an initial denaturation at $94^{\circ} \mathrm{C}$ for 2 minutes followed by 35 cycles of $94^{\circ} \mathrm{C}$ for 1 minute, $50^{\circ} \mathrm{C}$ for 1 minute, $72^{\circ} \mathrm{C}$ for one minute and finished with 5 minutes extension at $72^{\circ} \mathrm{C}$.

Size of amplified PCR products for all samples were checked using a 100 base pair (bp) DNA ladder (Roche, Penzberg, Germany), or in some instances a 123 base pair ladder (Invitrogen) on a 1.5\% agarose gel (photos of gels of all trialled primers are displayed in Figures 3.1 and 3.2). The amplified products were cleaned using exosap with $1-5 \mu \mathrm{L}$ of exo-sap per $25 \mu \mathrm{L}$ of PCR product. The purified PCR product was then sequenced on an AB13730 Genetic Analyzer by the Allan Wilson Centre Genome Service (Massey University, Palmerston North, New Zealand). Reverse compliments were sequenced to confirm reads for a number of samples.

\section{Sequence alignment and phylogenetic analysis}

DNA sequences were aligned using Geneious Pro 3.8.5 (Drummond et al., 2007). Before alignment the sequences were submitted to a BLAST search (http://www.ncbi.nlm.nih.gov/blast/) to check the expected marker had indeed been amplified. Samples that had been sequenced from forward and reverse directions were combined using Geneious Pro 3.8.5 (Drummond et al., 2007). Of the 105 sequences analysed in this study six were downloaded from GenBank, 30 were sequenced in Chapter Two and 69 were newly sequenced here. All new sequences will be deposited in GenBank upon publication.

Analyses were first performed individually for chloroplast ( $\operatorname{trn} L-F$ and $\operatorname{trnK}$ ) and nuclear (ITS) data sets and then on a concatenated data set (trnL-F, trnK and ITS). 
Some analyses were run on the freely available Bioportal (www.bioportal.uio.no). Congruence between the ITS and chloroplast datasets was assessed by visual assessment of the topologies and by running an homogeneity partition test in PAUP* (Swofford, 2002) with 100 replicates, TBR branch swapping and maximum of 1000 trees. Maximum Parsimony (MP) analyses were conducted using PAUP* v4.0b10 (Swofford, 2002). Due to the large number of most parsimonious trees (MPTs), MP analysis was performed in a two-step search strategy. First, multiple islands were searched with 10000 random addition sequence replicates, nchuck $=5$, chuckscore $=$ 1 and maxtrees $=10000$. The resulting trees were then swapped to completion with the same settings but chuckscore $=$ no. Support for clades was assessed using 1000 bootstrap replicates, 10 random addition replicates, and MAXTREES $=20000$ in PAUP*.

Maximum likelihood (ML) tests were conducted using Garli (Zwickl, 2006) using mostly default settings, except that 1000 bootstrap replicates were conducted and the model of evolution was altered to fit those selected by jModelTest (Posada, 2008). JModelTest was used to test the fit amongst 88 different models of different complexity. Models were selected using Akaike information weights criterion (AIC).

MrBayes v3.1.1 (Huelsenbeck, 2001) was used to conduct heuristic searches, also implementing the AIC model that was selected by jModelTest (Posada, 2008). Each dataset was analysed with four chains and either 7 or 10 million generations, in most cases until the standard deviation of split frequencies had fallen below 0.01 , which indicates the runs are stationary. The consensus trees were created excluding $10 \%$ of the trees as burn-in. Ten percent was selected as an appropriate amount to ensure that the runs had converged on a stationary distribution by considering the standard deviation of split frequencies and exploring the MrBayes output in Tracer v. 1.5 (Rambaut and Drummond, 2007). Fifty-percent majority rule consensus trees were created for all Bayesian analyses.

SplitsTree v4.10 (Huson and Bryant, 2006) was used to create neighbour net networks to explore conflicting signals both with the chloroplast and nuclear ITS datasets separately, and within the combined datasets. Outgroup samples were excluded from these analyses. 


\section{Biogeographic and character analysis}

Several character traits were mapped onto the nuclear chloroplast and combined Bayesian trees using MacClade v 4.08 (Maddison, 2001). Characters included geographical location (New Zealand, Australia, Europe) and herbaceous growth form (radicate vs. rhizomatous). No additional characters were mapped as analysis in Chapter Two showed that mapping ploidy levels was unfeasible for this study, and preliminary analysis showed that additional geographical characters were not informative and appeared to explain less of the topology of the phylogenetic trees than the large scale distribution (data not shown, three different scales were explored: 1. North or South Island, 2. west or east of the alpine fault, and 3. North Island, north of the South Island, middle of the South Island and south of the South Island).

\section{Results}

\section{DNA extractions and primer selection}

Large amounts of high quality DNA were extracted from 99 samples of Wahlenbergia, Campanula and Lobelia. Nanodrop concentrations measured ranged from $72.6 \mathrm{ng} / \mu 1$ to $2005.4 \mathrm{ng} / \mu \mathrm{l}$. Of the chloroplast markers trialled only four amplified across all four trial Wahlenbergia samples: psbM1-trnD, psbA-trnH, trnL$F$ and trnK-psbAR (gels in Figure 3.1, primer sequences and references Table 3.1). Of these $p s b M 1-t r n D$ and $p s b A$-trnH failed to sequence cleanly. Both $\operatorname{trn} L-F$ and $\operatorname{trnK}$ - $p s b A R$ (hereafter referred to as $\operatorname{trnK}$ ) amplified and sequenced readily, and contained some level of variation, so were selected for use in this study. Of the nuclear markers trialled, four amplified relatively cleanly across all four Wahlenbergia samples (Figure 3.2): the low-copy markers 'Actin', 'atpB' and Eif3E, and the nuclear ribosomal ITS. Note that 'Actin' and 'atpB' are tag names given to these primer pairs and do not refer the chloroplast regions commonly given these names (Steele et al., 2008). Both 'Actin' and 'atpB' sequenced relatively cleanly but contained only a limited amount of variation between sequences, mostly due to heterozygous positions, so were assessed as having too low a level of phylogenetic information to be useful. The third low-copy marker Eif3E could not be sequenced 
cleanly. The nuclear ribosomal ITS was a challenge to amplify and sequence and one base length indels were found to be present in a few samples, but was nevertheless used in this study. In some cases these sequences could still be used by obtaining the reverse sequence and creating contigs, but in other cases obtaining ITS sequences for certain samples had to be abandoned. Both of the mitochondrial markers trialled amplified and sequenced cleanly (Figure 3.2), but all four Wahlenbergia samples shared the identical haplotype for both markers, so were deemed of no use for phylogenetic analysis at the species level and were not pursued further.

\section{aDNA extractions}

DNA was successfully extracted from six herbarium samples using the aDNA method outlined above (four samples of $W$. littoricola subsp. littoricola (LITT_NSW_Buff1-4), one of $W$.preissii (PREI_WA_Mund) and one of $W$. tumidifructa (TUME_VIC_Black). Because the samples were brought back to a modern lab for PCR and preparation for sequencing there is a chance they may have been contaminated with Wahlenbergia DNA present in the modern lab. This appears unlikely however, as three distinct chloroplast haplotypes were sequenced from the six samples, one of which ( $W$. tumidifructa) had a unique haplotype in the dataset as a whole. Unfortunately no nrITS sequences were able to be generated for the aDNA samples, probably due to the small amount and lower quality of the DNA extracted compared to that extracted from more recently collected samples. 


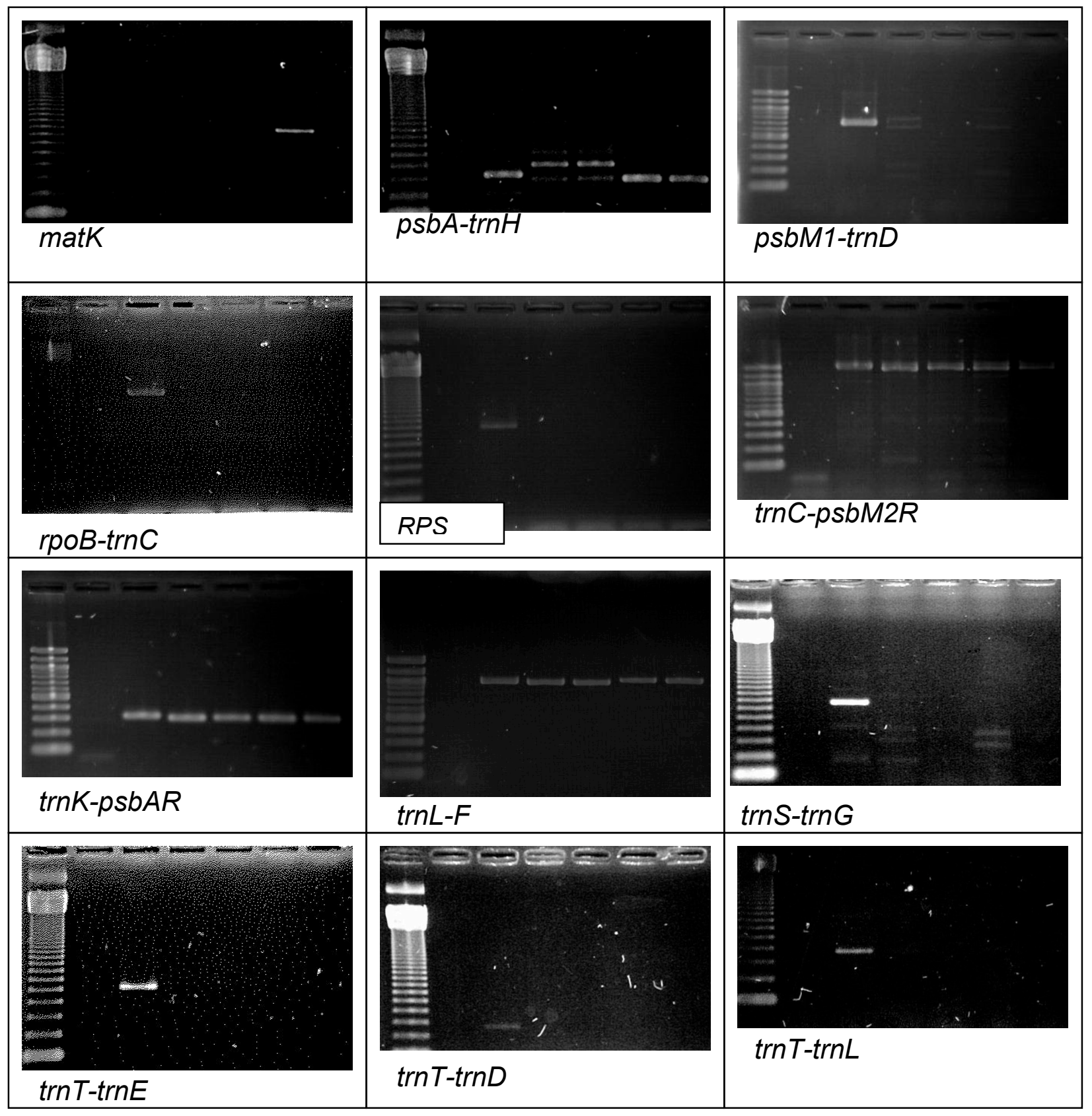

Figure 3.1 Photographs of gels showing chloroplast primers trialled. The ladders are either 123 or 100bp ladders. The columns for each from left to right are ladder, - ve control, + control, W. communis (COMM_NSW_Quea), $W$. violacea(VIOL_SI_Dun), $W$. albomarginata subsp. laxa (LAXA_SI_Cobb1) and W. albomarginatā subsp. albomarginata (ALBO_SI_Garv) except for matK in which the columns are reversed. Primer sequences and references are listed in Table 3.1. Explanation of tag names and voucher information can be found in Appendix 1. 

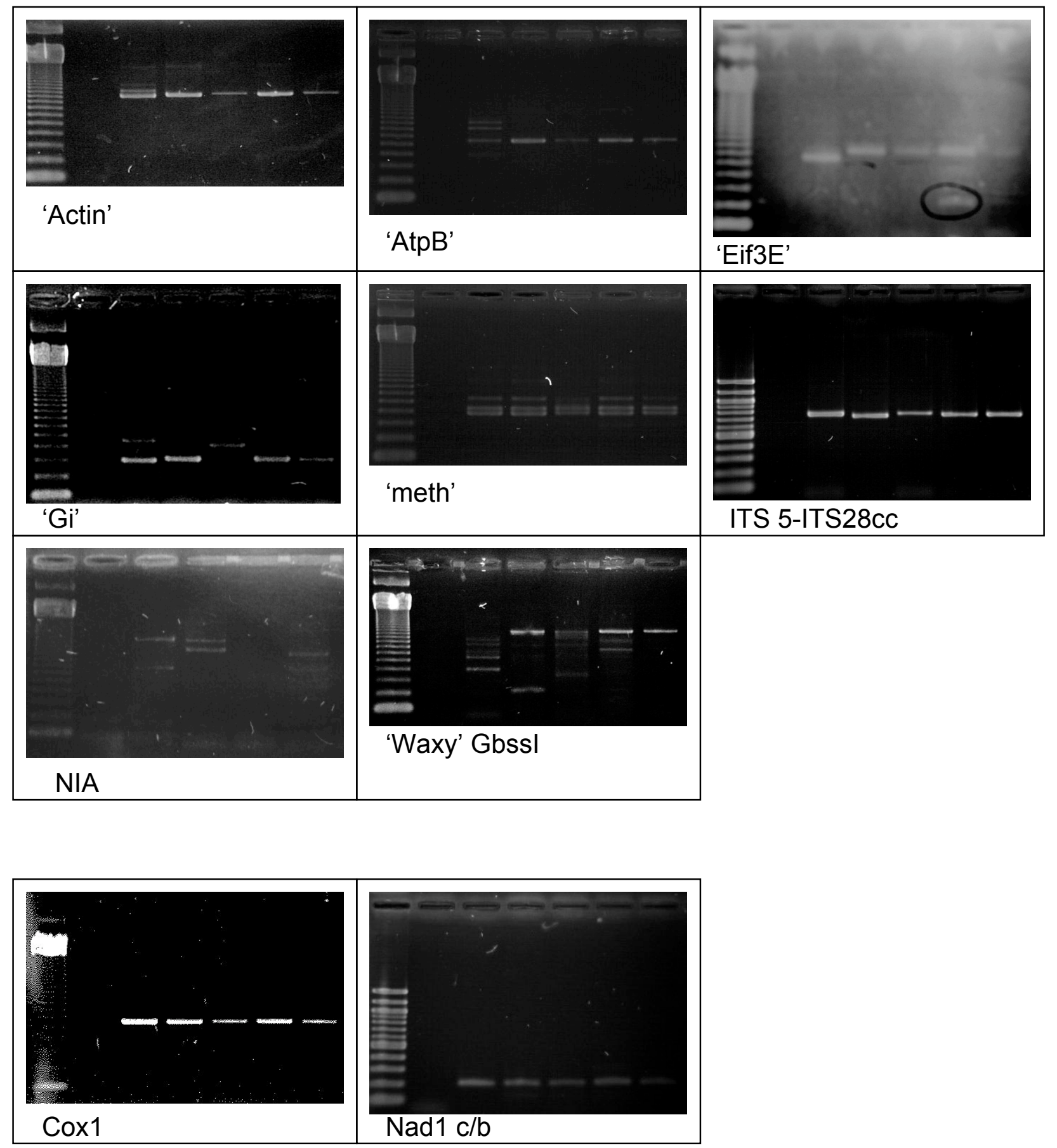

Figure 3.2 Photographs of gels showing nuclear (top) and mitochondrial (bottom) primers trialled. The ladders are either 123 or $100 \mathrm{bp}$ ladders. The columns for each are from left to right ladder, -ve control, + control, $W$. communis (COMM_NSW_Quea), W. violacea (VIOL_SI_Dun), W. albomarginata subsp. laxa (LAXA_SI_Cobb1) and W. albomarginata subsp. albomarginata (ALBO_SI_Garv). Primer sequences and references are listed in Table 3.2. Explanation of tag names and voucher information can be found in Appendix 1. 


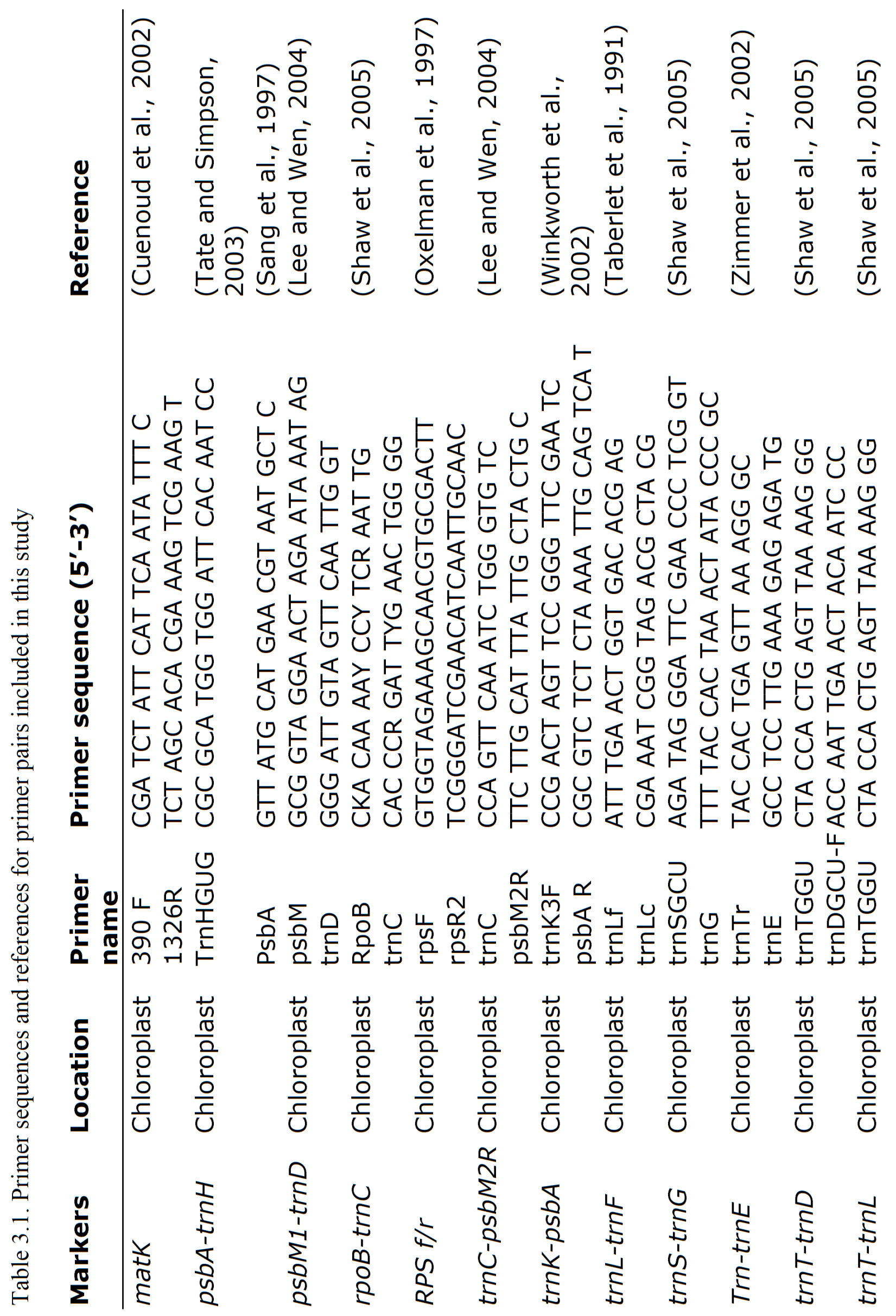




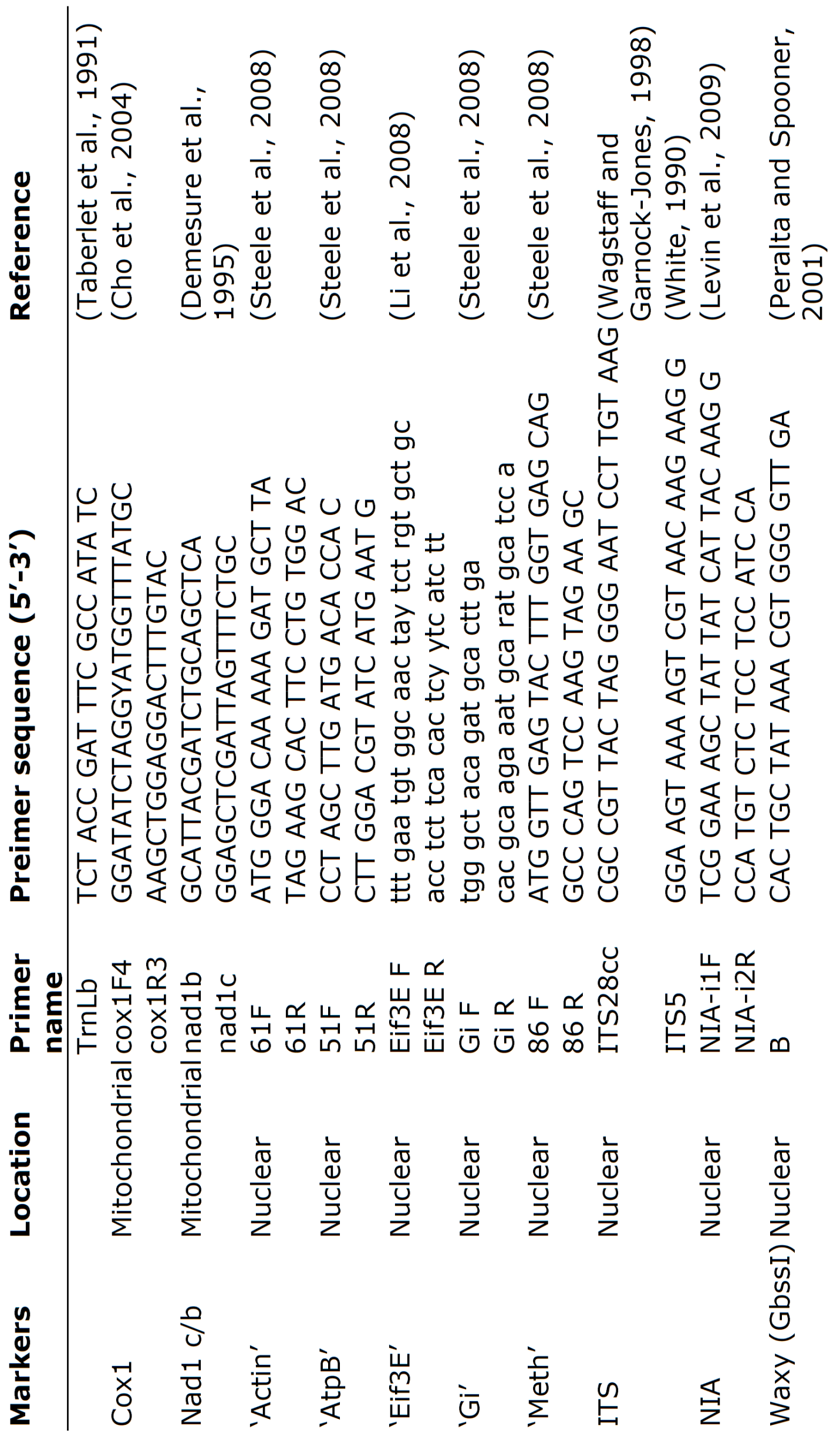




\section{ITS analysis}

The final ITS dataset contained 77 individuals (74 Wahlenbergia plus four outgroups) and was 816 characters long, 279 of which were variable and 133 parsimony informative. The model of evolution selected by jModelTest was GTR $+\mathrm{G}$ with base frequencies of variable sites: $A=17.6 \% \mathrm{C}=29.1 \% \mathrm{G}=31.4 \% \mathrm{~T}=21.9 \%$, scaled transitions and transversion substitutions in the dataset: $\mathrm{AC}=1.3169 \mathrm{AG}=$ $3.5376 \mathrm{AT}=2.0637 \mathrm{CG}=0.8530 \mathrm{CT}=1.1670$ and a gamma shape parameter $=$ 0.3879. The maximum parsimony (MP) analysis returned a tree with the best score of 400. The full number of most parsimonious trees was not recorded due to the sampling method, but was at least 10 000. The Bayesian analysis ran for 7 million generations, at which time the standard deviation of split frequencies was $<0.01$, which increases the likelihood that the runs had converged on a stationary distribution. The score of the best maximum likelihood (ML) tree was -3355.77 .

The MP, Bayesian and ML methods returned 50\% majority rule consensus trees with similar topologies, differing only in that some poorly supported clades evident in the Bayesian analysis were missing from the MP and ML trees. The (Australasian) Wahlenbergia samples formed a highly supported clade (1.0 posterior probability, 100\% ML bootstrap, 100\% MP bootstrap; hereafter 1.0/100/100 see Figure 3.3) separate from the outgroup samples (European Wahlenbergia species were not included in the ITS dataset). Within this clade relationships were poorly resolved, indicated by the large polytomy containing eight clades. Within this polytomy the clades that contained fewer samples were more highly supported than those containing more samples, which generally only had low support from the Bayesian analysis and were absent from the MP or ML trees.

The New Zealand species fell into three clades: first, a poorly supported one containing all of the New Zealand rhizomatous species (clade NZ2 0.75/<50/51); secondly, a clade with most of the New Zealand radicate species (clade NZ1-, $0.94 / 75 / 70$, and thirdly a well-supported clade containing two (radicate) $W$. ramosa samples (1.0/85/87, Figure 3.3). 


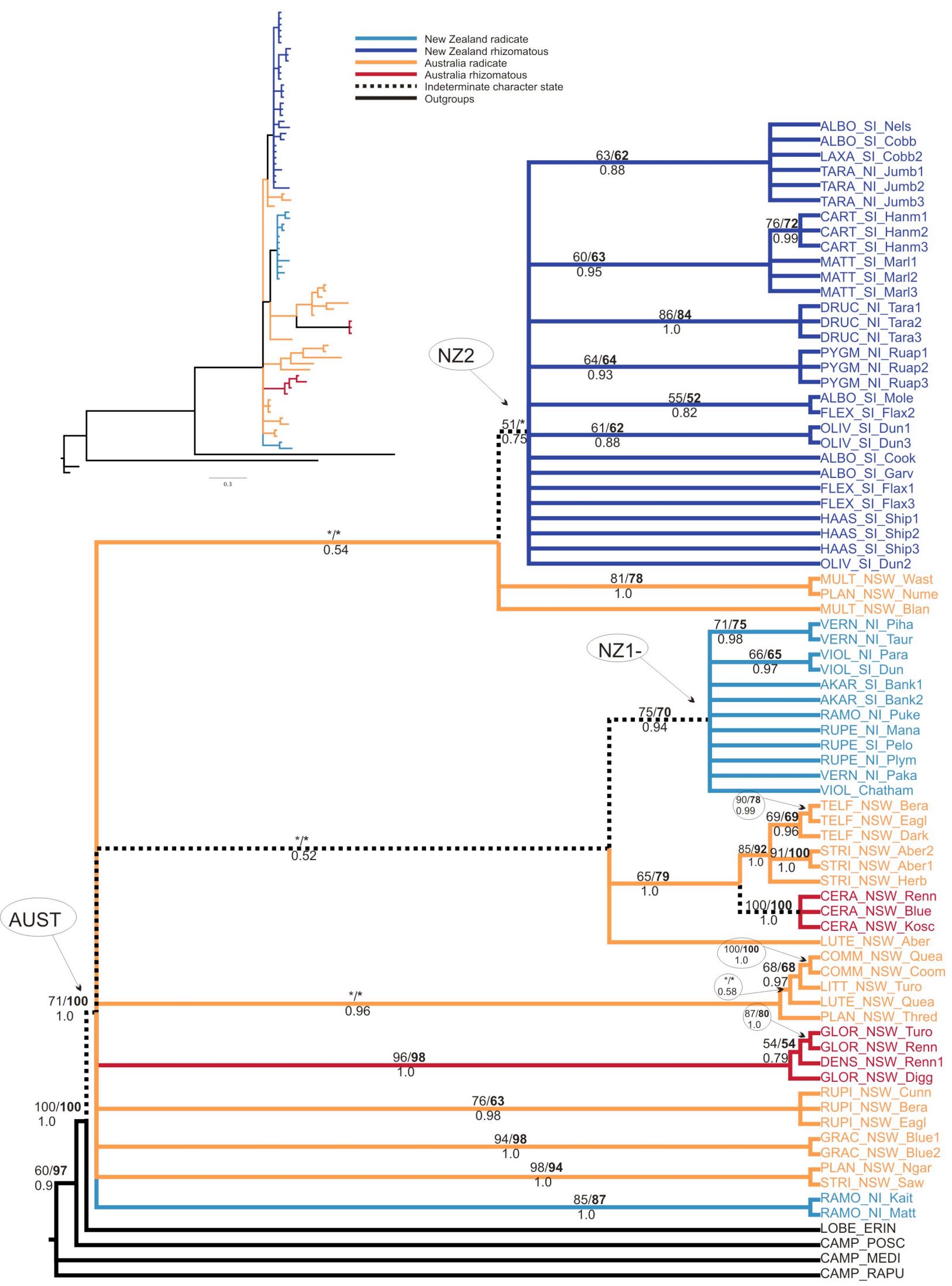

0.3

Figure 3.3 Bayesian 50\% majority rule consensus tree of Wahlenbergia (colours) and outgroup (black) based on the ITS dataset. Inset is the phylogram of the same tree. Numbers above branches are bootstrap support from the ML/MP analyses. Numbers below branches are posterior probability. AUST = Australasian Wahlenbergia clade, NZ1- = most samples of New Zealand species with a radicate growth form. NZ2 $=$ New Zealand species with a rhizomatous growth form. See Appendix 1 for an explanation of the tag names and voucher information. 
None of the New Zealand radicate species formed monophyletic groups within clade NZ1 (Figure 3.3), and samples from each species are either unresolved (e.g. $W$. akaroa and $W$. rupestris), paraphyletic (e.g. W. littoricola. subsp. vernicosa and $W$. violacea) or polyphyletic (e.g. W. ramosa). Thus, relationships within the W. gracilis complex are unresolved, and even the sub-tropical $W$. littoricola. subsp. vernicosa does not form its own lineage. Genetic distances among the New Zealand radicate species were low, as evidence by the $\mathrm{p}$ values (range $0-0.025$, Table 3.2 ). Interestingly the Australian $W$. littoricola subsp. littoricola sample does not group with the New Zealand $W$. littoricola subsp. vernicosa samples.

The only New Zealand rhizomatous species to form a monophyletic group within clade NZ2 (Figure 3.3) was $W$. cartilaginea $(0.99 / 76 / 72)$, although a few subspecies did too e.g. W. albomarginata subsp. drucei (1.0/86/84) and W. pygmaea subsp. pygmaea (0.93/64/64). The majority of species/subspecies relationships were either unresolved (e.g. W. pygmaea subsp. tararua, W. matthewsii, W. congesta subsp. haastii) or paraphyletic (e.g. W. albomarginata subsp. albomarginata, W. albomarginata subsp. olivina, W. albomarginata subsp. flexilis). Thus, while the subspecies of $W$. albomarginata and $W$. pygmaea often formed monophyletic groups the species themselves did not. Genetic distances among New Zealand rhizomatous species were also low, with a range in p distance values of 0 to 0.015 (Table 3.2).

Although large-scale relationships of the Australian species were largely unresolved, in a number of cases all samples from the same species formed well-supported clades (e.g. W. ceracea, W. communis, W. gracilis and the two newly described Australian species $W$. rupicola and $W$. telfordii). However, the majority of samples from one species are either paraphyletic (e.g. W. multicaulis and $W$. gloriosa) or polyphyletic (e.g. W. planiflora, W. luteola and W. stricta). While the newly described $W$. telfordii formed a highly supported clade with the most of the samples of $W$. stricta and all of the samples of $W$. ceracea, the position of the newly described $W$. rupicola was unresolved. The Australian rhizomatous species fall in two separate clades, and there is no relationship between them and the New Zealand rhizomatous species. The genetic distances among Australian species was slightly higher than those among the New Zealand species, among the Australian rhizomatous species $\mathrm{p}$ distance values 
ranged from 0 and 0.054 and among the Australian radicate species p distance values ranged from 0 and 0.065 (Table 3.2)

The neighbour net network (Appendix 2.1) revealed high levels of conflicting signal in the ITS dataset, particularly regarding the Australian radicate samples. Also, as expected due to their low genetic variation for the ITS marker, the New Zealand rhizomatous and radicate species have star-like topologies in the network.

\section{Chloroplast markers analysis}

The final chloroplast dataset contained 105 individuals (96 Wahlenbergia plus 9 outgroups) and was 1131 characters long, 889 characters from the $\operatorname{trnL}-F$ marker and 242 characters from the $\operatorname{trn} K$ marker. There were 370 variable characters, 241 of which were parsimony informative. The 10 samples that were sequenced only for either $\operatorname{trn} L-F$ or $\operatorname{trn} K$ were coded as missing data in the concatenated alignment. The model of evolution selected by jModelTest was GTR $+\mathrm{G}$ with base frequencies of variable sites $\mathrm{A}=32.6 \% \mathrm{C}=17.4 \% \mathrm{G}=18.7 \% \mathrm{~T}=31.3 \%$, scaled transitions and transversion substitutions in the dataset: $\mathrm{AC}=1.0481 \mathrm{AG}=0.6020 \mathrm{AT}=0.1343 \mathrm{CG}$ $=1.2275 \mathrm{CT}=0.8801$ and a gamma shape parameter $=0.9499$. The parsimony analysis returned a tree with the best score of 531. The full number of most parsimonious trees was not recorded due to the sampling method, but was at least 10 000. The Bayesian analysis ran for 7 million generations, by which time the standard deviation of split frequencies was below the 0.01 recommended. The score of the best ML tree was -4575.56 . The MP, Bayesian and ML methods returned 50\% majority rule consensus trees with very similar topologies, differing only in that the ML tree contained one clade not supported by the MP or Bayesian trees (data not shown, discussed further below) and that one poorly supported clade evident in the Bayesian analysis was missing from the MP and ML trees. The Wahlenbergia samples (excluding $W$. hederacea (HEDE_Europe), the placement of which is discussed in more detail in Chapter Two of this thesis) formed a highly supported clade (1.0/100/100) separate from the outgroup samples (Figure 3.4). Within this Wahlenbergia clade, the European $W$. lobelioides (LOBE_Europe) and the Victorian W. tumidifructa (TUME_VIC_Black) are successively sister to the remainder 


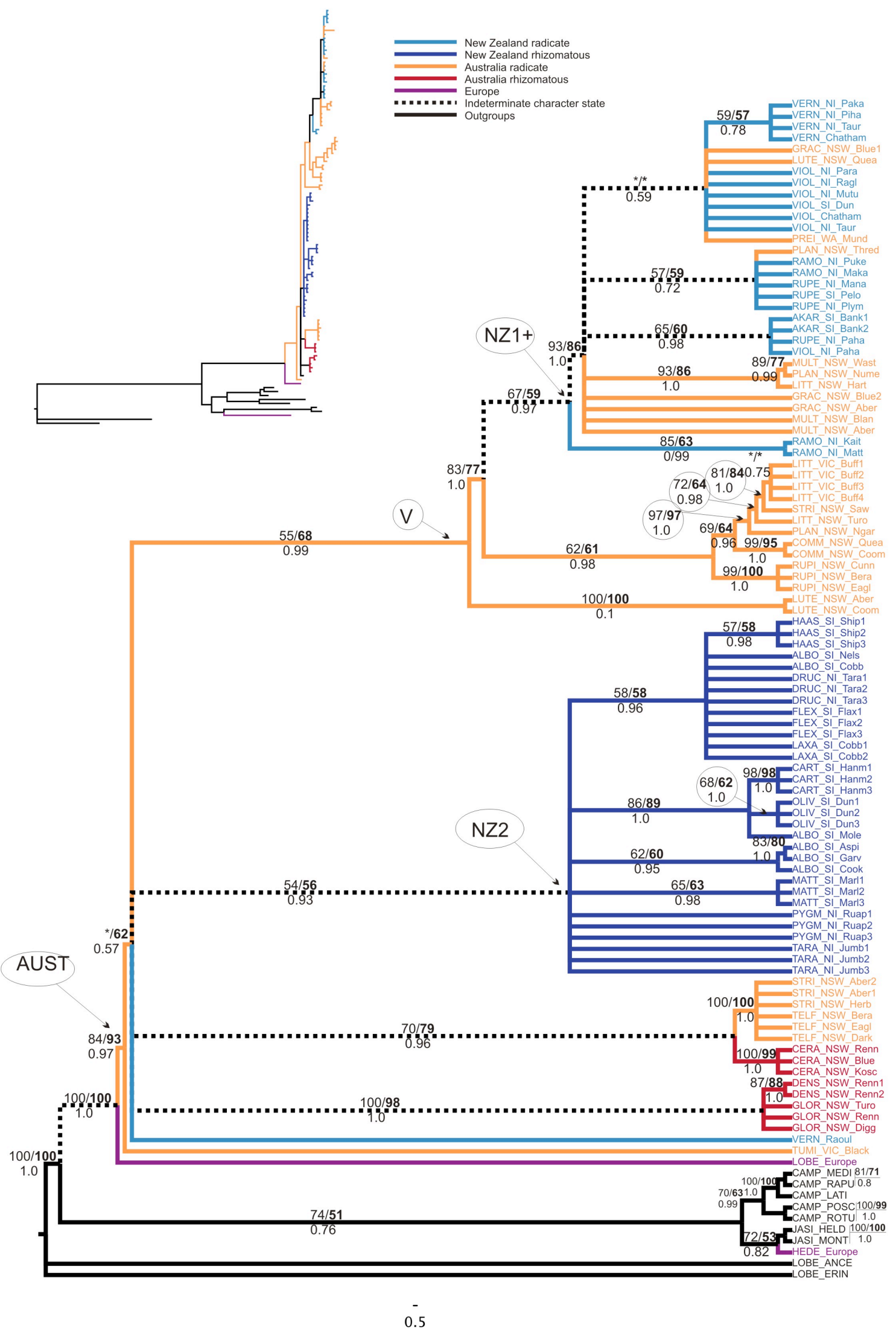

Figure 3.4 Bayesian 50\% majority rule consensus tree of Wahlenbergia (colours) and outgroup (black) based on the $\operatorname{trn} L-F+\operatorname{trn} K$ dataset. Inset is the phylogram of the same tree. Numbers above branches are bootstrap values (\%) from the ML/MP analyses. Number below branches are pp values. AUST $=$ Australasian Wahlenbergia clade, $\mathrm{NZ1}+=$ (mostly) New Zealand species with a radicate growth form. NZ2 = New Zealand species with a rhizomatous growth form. $\mathrm{V}=$ clade VERN_Raoul is sister to in ML analysis. See Appendix 1 for an explanation of the tag names and voucher information. 
of the Australasian Wahlenbergia. Within this main Australasian clade (0.97/84/93), the topology and support values are similar to the ITS tree in that relationships between species are generally poorly resolved, even though some species/subspecies are resolved as monophyletic.

Similar to the ITS tree the New Zealand radicate species do not form a monophyletic group, although all but one of them fall into the same clade (labelled NZ1+ in Figure $3.40 .97 / 67 / 59)$. Like the ITS tree, none of the New Zealand radicate species within this clade are themselves monophyletic. Wahlenbergia akaroa is unresolved, and the remainder (i.e., W. littoricola subsp. vernicosa, $W$. violacea, $W$. ramosa and $W$. rupestris) are all polyphyletic. The $W$. littoricola subsp. vernicosa samples mostly fall in a moderately supported (0.78/5759/) clade of their own and are polyphyletic only due to the placement of the $W$. littoricola subsp. vernicosa sample collected from Raoul Island (VERN_Raoul), the position of which is unresolved. In the MP and Bayesian analysis this sample formed part of a large unresolved polytomy, yet in the ML tree it is grouped as sister to clade V in Figure 3.4, with 57\% ML bootstrap support. It is clear the Australian $W$. littoricola samples do not form a clade with their proposed conspecific New Zealand $W$. littoricola subsp. vernicosa samples however, as these all fall in a separate clade. Genetic distances among the New Zealand radicate species were low, as evidenced by p distances (range $0-0.008$ Table 3.2).

Like the ITS tree, the New Zealand rhizomatous species form a clade (NZ2 $0.96 / 54 / 56$ ) and three species are resolved as monophyletic (i.e., $W$. cartilaginea (1.0/98/98), W. congesta subsp. haastii $(0.98 / 57 / 58)$ and $W$. matthewsii $(0.98 / 65 / 63))$. Additionally a few subspecies grouped in clades (e.g. $W$. albomarginata subsp. olivina, W. pygmaea subsp. drucei, W. pygmaea subsp. pygmaea and $W$. pygmaea subsp. tararua). The remainder were either unresolved (W. albomarginata subsp. flexilis and W. albomarginata subsp. laxa,) or polyphyletic (W. albomarginata subsp. albomarginata). Genetic distances are also low among the New Zealand rhizomatous species ( $\mathrm{p}$ distances range from 0 to 0.009 Table 3.2). 
Although large-scale relationships of the Australian species were (like the ITS tree) largely unresolved in the chloroplast tree, in a number of cases all samples from the same species formed well-supported clades on their own (e.g. W. ceracea (1.0/100/99), W. densifolia (1.0/87/88), W. communis (1.0/99/95) and the newly described species $W$. rupicola (1.0/99/100)) or together with another species (e.g., $W$. gloriosa and $W$. densifolia (1.0/100/98), or $W$. telfordii and $W$. stricta (1.0/100/100). However, the majority of samples from one species are either unresolved (e.g. $W$. telfordii), paraphyletic (e.g. W. gloriosa, W. gracilis and W. multicaulis) or polyphyletic (e.g. W. luteola, W. planiflora, W. littoricola subsp. littoricola and $W$. stricta) within the large clade comprising most of the NZ and Australian radicate species. Interestingly the four Australian $W$. littoricola subsp. littoricola samples extracted from herbarium specimens (LITT_NSW_Buff1-4) formed a highly supported clade, which also includes a sample identified as W. stricta (STRI_NSW_Saw) and an additional recently collected $W$. littoricola (LITT_NSW_Turo) sample. The voucher specimen for the $W$. stricta sample is rather lacking however (it is a poor specimen with few leaves and only one shrivelled flower), and I am not confident in my identification (i.e. there is a chance it could in fact be a $W$. littoricola). A sample identified as $W$. planiflora (PLAN_NSW_Ngar) is sister to this clade, and the identification of this specimen at least as not a $W$. littoricola is believable, as its flowers appear rotate (lacking a corolla tube), whereas $W$. littoricola flowers are generally shortly campanulate (Smith, 1992). The other sample identified as $W$. littoricola in this dataset (LITT_NSW_Hart), which does not group with the other samples mentioned above, is another recent collection, and again has a rather poor voucher specimen (few leaves, solo flower), making confirmation of the identification impossible. As in the ITS analysis the Australian species exhibit greater genetic variation in the chloroplast sequences than the New Zealand Wahlenbergia based on p distance values (a range of 0 to 0.025 among the Australian radicates, and 0 to 0.014 among the Australian rhizomatous Table 3.2).

The neighbour net network (Appendix 2.2) revealed less conflicting signal than the ITS neighbour net network, though conflict still is present. The main split in the network separates the majority of the NZ and Australian radicate species (excluding $W$. luteola, W. stricta, W. telfordii, $W$. tumidifructa and $W$. vernicosa from Raoul 
Island) from the rhizomatous species, with two samples of $W$. luteola exactly intermediate between these groups.

\section{Analysis of concatenated dataset}

The homogeneity partition test showed the nuclear vs. chloroplast alignments to be significantly incongruent $(\mathrm{p}=0.01)$. However, this test can be highly inaccurate even when the topologies of trees are congruent (Reeves et al., 2001; Yoder et al., 2001) although in this case visual assessment of the topologies does reveal a level of incongruence. Most of the incongruence appears to be "soft" incongruence, meaning that a particular relationship was resolved in one dataset but not in the other. An example of this is the $W$. littoricola subsp. vernicosa samples all of which are in the New Zealand radicate clade in the ITS

Table 3.2 Uncorrected $p$ distances calculated from the ITS and combined trnL$F+\operatorname{trn} K$ datasets. Readings for the New Zealand radicate group were calculated excluding the $W$. vernicosa sample from Raoul Island.

\begin{tabular}{|c|c|c|c|c|c|c|}
\hline & $\begin{array}{l}\text { Species } \\
\text { group }\end{array}$ & $\begin{array}{l}\text { Average } \\
\text { uncorrected } \\
\text { p distance }\end{array}$ & $\begin{array}{l}\text { Standard } \\
\text { deviation }\end{array}$ & $\begin{array}{l}\text { Minimum } \\
\text { uncorrected } \\
\text { p distance }\end{array}$ & $\begin{array}{l}\text { Maximum } \\
\text { uncorrected } \\
\text { p distance }\end{array}$ & $\begin{array}{l}\text { Samples with } \\
\text { maximum } \\
\text { uncorrected p } \\
\text { distance }\end{array}$ \\
\hline \multirow[t]{4}{*}{ ITS } & $\begin{array}{l}\text { New } \\
\text { Zealand } \\
\text { radicate }\end{array}$ & 0.008 & 0.006 & 0 & 0.025 & $\begin{array}{l}\text { RAMO_NI_Puke } \\
\text { RUPE_SI_Pelo }\end{array}$ \\
\hline & $\begin{array}{l}\text { New } \\
\text { Zealand } \\
\text { rhizomatous }\end{array}$ & 0.004 & 0.003 & 0 & 0.015 & $\begin{array}{l}\text { CART_SI_Hamn1 } \\
\text { OLVE_SI_Dun2 }\end{array}$ \\
\hline & $\begin{array}{l}\text { Australian } \\
\text { radicate }\end{array}$ & 0.029 & 0.014 & 0 & 0.065 & $\begin{array}{l}\text { LUTE_NSW_Quea } \\
\text { STRI_NSW_Aber2 }\end{array}$ \\
\hline & $\begin{array}{l}\text { Australian } \\
\text { rhizomatous }\end{array}$ & 0.029 & 0.023 & 0 & 0.054 & $\begin{array}{l}\text { CERĀ_NSW__Renn } \\
\text { GLOR_NSW_Renn }\end{array}$ \\
\hline \multirow[t]{4}{*}{$\begin{array}{l}\operatorname{trnL}-F \\
+ \\
\operatorname{trnK}\end{array}$} & $\begin{array}{l}\text { New } \\
\text { Zealand } \\
\text { radicate }\end{array}$ & 0.003 & 0.002 & 0 & 0.008 & $\begin{array}{l}\text { RAMO_NI_Kai } \\
\text { VERN_Chatham }\end{array}$ \\
\hline & $\begin{array}{l}\text { New } \\
\text { Zealand } \\
\text { rhizomatous }\end{array}$ & 0.003 & 0.002 & 0 & 0.009 & $\begin{array}{l}\text { ALBO_SI_Aspi } \\
\text { CART_SI_Hanm3 }\end{array}$ \\
\hline & $\begin{array}{l}\text { Australian } \\
\text { radicate }\end{array}$ & 0.013 & 0.006 & 0 & 0.025 & $\begin{array}{l}\text { LUTE_NSW_Quea } \\
\text { STRI_NSW_Herb }\end{array}$ \\
\hline & $\begin{array}{l}\text { Australian } \\
\text { rhizomatous }\end{array}$ & 0.008 & 0.006 & 0 & 0.014 & $\begin{array}{l}\text { CERĀ_NSW_Blue } \\
\text { DENS_NSW_Renn1 }\end{array}$ \\
\hline
\end{tabular}

tree (clade NZ1 - in Figure 3.3), and two of the three samples form a moderately supported clade within this. In contrast all of the $W$. littoricola subsp. vernicosa samples form a moderately supported clade in the chloroplast tree (Figure 3.4) 
(excluding the Raoul Island sample which was not included in the ITS dataset). A rare example of a highly supported "hard" incongruence between the datasets is the relationship of the $W$. cartilaginea samples to the $W$. matthewsii samples. In the ITS tree a clade containing samples from these species has moderate support (0.95/60/63), but this clade is absent from the $\operatorname{trn} L-F+\operatorname{trn} K$ tree, where instead a clade containing the $W$. cartilaginea samples, the $W$. albomarginata subsp. olivina samples and one $W$. albomarginata subsp. albomarginata sample has high support (1.0/86/89). The neighbour net network (Appendix 2.3) of the concatenated dataset also revealed a high level of conflicting signals within the dataset, though this contained conflict within the individual ITS and chloroplast datasets, and it is therefore difficult to determine the source of the conflict. I nevertheless proceeded with analysis of the concatenated dataset, with the caveat that all results should be treated with caution due to the incongruence discussed above.

\section{Combined ITS, trnK and trnL-F analysis}

The final concatenated dataset contained 105 samples and was 1947 characters long, 1131 (58\%) from the chloroplast and 816 (42\%) from the nuclear genome. There were 649 variable characters and 374 parsimony informative ones. The majority of the variable characters were from the chloroplast dataset $(370,57 \%)$, though this was in proportion with the greater number of characters contributed by the chloroplast dataset. The majority of the parsimony informative characters were also from the chloroplast data (64\%), which was a slightly higher proportion than its contribution of characters. The 28 samples that were sequenced only for chloroplast markers were coded as missing data for the ITS characters in the concatenated alignment. It was not necessary to estimate parameters independently between data partitions as both the nuclear and chloroplast datasets were best estimated by the same model of evolution $(\mathrm{GTR}+\mathrm{G})$. The parsimony analysis returned a tree with the best score of 1005. The full number of most parsimonious trees was not recorded due to the sampling method, but was at least 10 000. The Bayesian analysis ran for 10 million generations, by which time the standard deviation of split frequencies was at 0.02 , which is higher than the point recommended to stop the analysis, but it seemed unlikely to drop below the threshold of 0.01 within an acceptable timeframe. The score of the best ML tree was -7947.01 . 


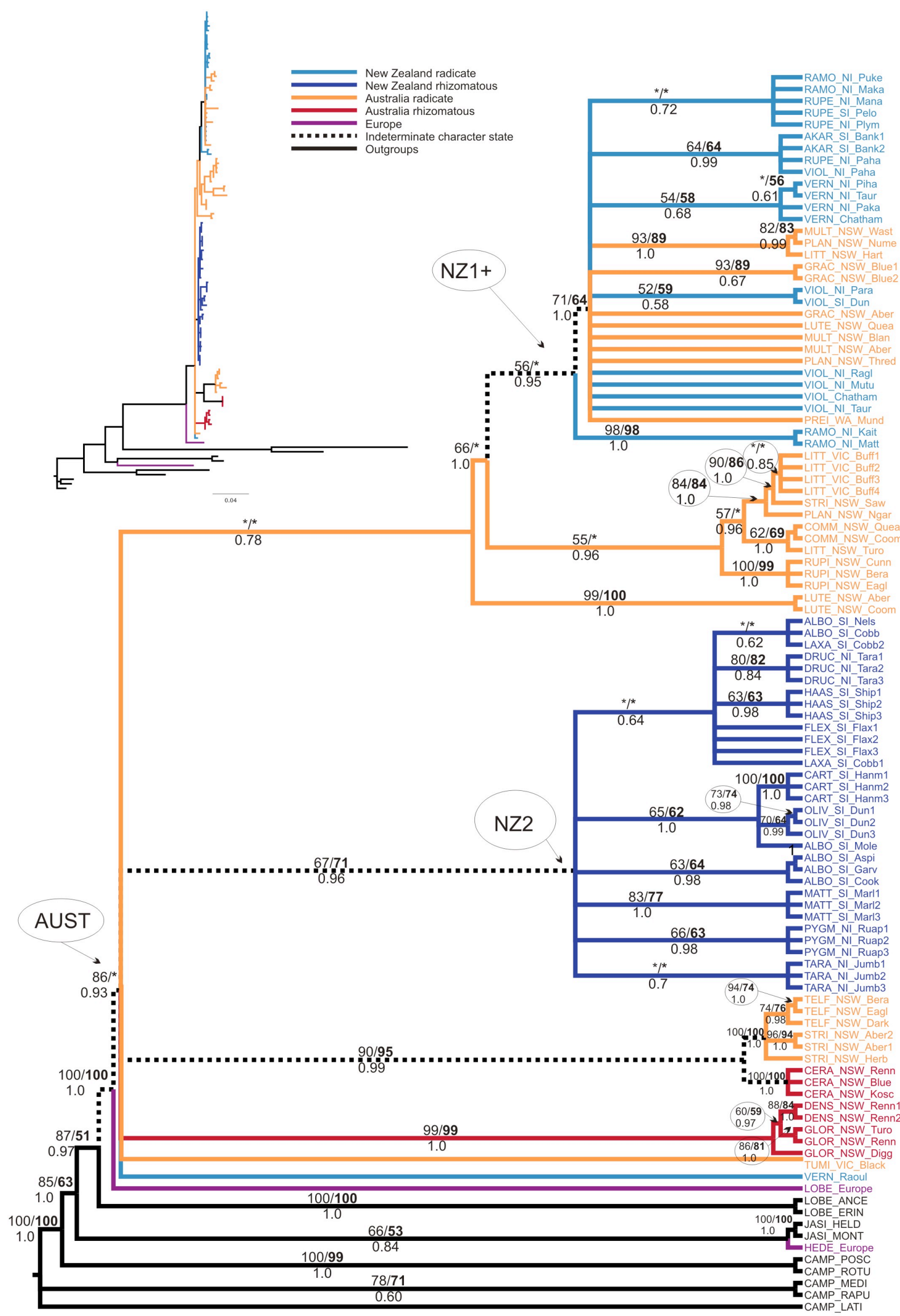

0.04

Figure 3.5 Bayesian 50\% majority rule consensus tree of Wahlenbergia (colours) and outgroup (black) based on the combined ITS $+\operatorname{trn} L-F+\operatorname{trn} K$ dataset. Inset is the phylogram of the same tree. Numbers above branches are bootstrap values (\%) from the ML/MP analyses. Number below branches are pp values. AUST $=$ Australasian Wahlenbergia clade, NZ1 $=$ (mostly) New Zealand species with a radicate growth form, NZ2 $=$ New Zealand species with a rhizomatous growth form. See Appendix 1 for an explanation of the tag names and voucher information. 
The MP, Bayesian and ML methods returned 50\% majority rule consensus trees with relatively similar topologies, differing in that a number of moderately and poorly supported clades evident in the Bayesian analysis were missing from the MP and ML trees, and one clade was present in the MP tree but absent from the Bayesian and ML analyses ( $W$. tumidifructa was placed as sister to all other Australasian Wahlenbergia with 53\% bootstrap support, data not shown). The Wahlenbergia samples (again excluding Wahlenbergia hederacea) formed a highly supported clade (1.0/99/99) separate from the outgroup samples (Figure 3.5). As in the chloroplast tree, within this Wahlenbergia clade the European $W$. lobelioides (LOBE_Europe) and the Victorian W. tumidifructa (TUME_VIC_Black) were sister to the remainder of the Australasian Wahlenbergia (only supported by 54\% bootstrap support in the MP analysis, data not shown). In general the areas of incongruence between the ITS and chloroplast trees have been resolved in favour of the relationships present in the chloroplast tree, probably because the chloroplast tree was reconstructed using a greater number of characters (and higher percentage of the parsimony informative characters) and generally had slightly higher support values than the ITS tree. Unsurprisingly the pattern of low resolution at the among-species level, present in both the nuclear and chloroplast trees, is repeated in the concatenated tree. More species/subspecies are recovered as monophyletic in the concatenated tree compared to either of the nuclear or chloroplast trees though, as information resolving different species/subspecies was present in the different trees.

\section{Discussion}

The central aim of this chapter was to generate the first phylogeny of the Australasian Wahlenbergia, and to use this to test the current taxonomy. The phylogenies presented here are poorly resolved, which limits their usefulness in the role of exploring relationships within Wahlenbergia. Nevertheless some parts of the trees, generally more towards the tips of the branches, are resolved giving some information at the species level.

\section{Evolution of Australasian Wahlenbergia}

In the previous chapter in this thesis the Australasian Wahlenbergia were shown to 
be monophyletic, and two introductions from Australia to New Zealand of

Wahlenbergia were indicated, one leading to the rhizomatous radiation, and the other to the radicate radiation. With greater sampling of both Australian and New Zealand individuals in this chapter that clear pattern is somewhat obscured. It still appears most likely that the New Zealand rhizomatous species are the result of a single introduction from Australia, but there is no support for Smith's (1992) hypothesis that the New Zealand rhizomatous species are derived from the Australian rhizomatous species. In fact, it is unclear which Australian species they may be most closely related to as there is a general lack of support and resolution in the backbone of the Australasian Wahlenbergia phylogenies presented here (Figures 3.3-3.5). Unfortunately this study did not include any samples of the Tasmanian rhizomatous $W$. saxicola, which was even considered conspecific with the New Zealand rhizomatous species at one time, and this may represent the sister species to the New Zealand rhizomatous clade.

Additionally, and in contrast to the findings of Chapter Two of this thesis, there is also no clear New Zealand radicate clade. All the New Zealand radicate species appear to be very closely related to each other and to certain Australian radicate species, particularly $W$. gracilis, $W$. multicaulis and $W$. planiflora. In fact, some individuals from different species from Australia and New Zealand have identical chloroplast haplotypes (e.g. the Australian W. gracilis (GRAC_NSW_Blue1) and a number of the New Zealand $W$. violacea samples (e.g. VIOL_NI_Para). This low level of sequence variation suggests recent evolution, which was also indicated in the last chapter where I found the New Zealand radicate Wahlenbergia had radiated about 0.5 mya. Additionally there is very low sequence divergence within the New Zealand radicate species as indicated by low average uncorrected p distances (e.g. 0.003 for the chloroplast dataset and 0.008 of the nrITS).

Low genetic diversity exhibited by a range of chloroplast and nuclear markers has been found in a number of other New Zealand plant genera, which have also probably radiated within the last 5 my (e.g. Plantago (Tay et al., 2010), Myosotis (Winkworth et al., 2002a) and Ranunculus (Lockhart et al., 2001)). Recent evolution, combined with the geological and climatic conditions during the last $5 \mathrm{my}$, has been implicated in causing this low level of diversity. Species with conspicuous 
morphological and ecological differences exhibit little or no genetic differentiation with both nuclear and chloroplast loci, suggesting that differentiation has occurred over a relatively short period of time. Examples of recent diversification in New Zealand include the Gnaphalieae (Smissen et al., 2004), Ourisia (Meudt and Simpson, 2006), Ranunculus (Lockhart et al., 2001) and the hebe complex (Wagstaff and Garnock-Jones, 1998, 2000).

It can be very challenging to identify species in recently evolved/ evolving lineages, as insufficient time may have passed for reproductive isolation and gene-tree monophyly to have become fixed (Shaffer and Thomson, 2007). Furthermore, Hudson and Coyne (2002) demonstrated that reciprocal monophyly for a pair of species takes from 4 to $7 \mathrm{Ne}$ generations for $50 \%$ of nuclear genes sampled (where $\mathrm{Ne}$ is the historically effective population size of each descendant taxon) to 9 to 12 Ne generations for $95 \%$ of sampled genes. Historical and current population sizes are usually unknown, but it seems likely that reciprocal monophyly for most genes will require from tens of thousands to millions of generations, implying a long waiting time for monophyly to evolve even in completely isolated lineages (Shaffer and Thompson, 2007). As a recently diverged lineage in which hybridisation is known (Smith 1992; Petterson, 1997b) species of Australasian Wahlenbergia may not have evolved reciprocal monophyly. Thus, despite the lack of monophyly for a number of currently accepted species of Australasian Wahlenbergia in this chloroplast and nuclear DNA sequence analysis, other lines of evidence (e.g. morphological, cytological etc) should be considered before the species are rejected.

\section{Testing the New Zealand Wahlenbergia taxonomy}

\section{New Zealand radicate species}

The New Zealand radicate species are not recovered as a monophyletic group in any of the analyses (MP, ML and Bayesian) for either of the datasets (chloroplast or nuclear) and none of the species are recovered as monophyletic. The relationships among samples from the $W$. gracilis complex are unresolved in all analyses. The ITS tree is extremely poorly resolved with regards the $W$. gracilis complex and the chloroplast tree is not much better. The only clade that receives high support in both the ITS and chloroplast trees is that of two W. ramosa individuals (RAMO_NI_Kait 
and RAMO_NI_Matt) that is separate from the remaining New Zealand radicate species. These two individuals were collected from hills in the Wellington region; there are no other obvious morphological or geographical characters that distinguish them.

None of the temperate radicate species (the $W$. gracilis complex) therefore have evolved monophyly according to three DNA sequence markers, but does this mean they shouldn't be considered species? Recall that the morphological differences used to separate them are largely based on floral differences regarding colour, size, and/or degree of petal overlap (Petterson, 1997b). In addition, all have the same chromosome number $2 n=72$, and all except $W$. akaroa have largely overlapping distributions on both the North and South Islands. Thus, the morphological differences are not great and are often difficult to score, and there is no additional genetic, cytological, or ecological data to suggest there are four separate lineages within the $W$. gracilis complex. The ability of several of the $W$. gracilis complex to hybridise further complicates the picture. Plants that are hybridising, even if they are in independent lineages, will take even longer to evolve monophyly than those that have developed complete reproductive isolation (Shaffer and Thompson 2007). Information about hybridisation among the $W$. gracilis complex has so far been restricted to observational studies, recordings of purported hybrid swarms based on field observations of morphologically intermediate individuals growing within proximity of 'pure' populations of the parent species (Petterson, 1997b). If extensive hybridisation and gene flow are occurring, it could provide additional evidence that this complex is effectively a single lineage, and recognition of species within this lineage is therefore not appropriate. It would be useful to undertake controlled crosspollinations in a glass house setting to determine whether hybrids do in fact set viable seed, and whether there is any difference in the viability of seeds formed from parents of the same species vs. different species. This is key to whether there are any barriers to cross-pollination between these four radicate species, which would help to determine their taxonomic status. 
The position of $W$. littoricola subsp. vernicosa

$W$. littoricola subsp. vernicosa, the one New Zealand radicate species to have a different chromosome count from the other New Zealand radicate species ( $2 n=54$ vs. $2 n=72$ (Petterson et al., 1995)), is found in subtropical areas of northern New Zealand, the Chatham Islands, plus several islands in the Pacific (Petterson, 1997b). The phylogenetic analysis conducted in this study does not support the reduction of $W$. vernicosa to a subsp. of $W$. littoricola (de Lange and Cameron, 1999). Evidence to support the subspecies rank could have included samples from the two subspecies forming a monophyletic group, or given the lack of resolution at least samples from both subspecies belonging to the same unresolved grade. However, in both the chloroplast and ITS trees the $W$. vernicosa samples are in a quite separate clade from the $W$. littoricola samples. Both the chloroplast and ITS trees resolve other Australian and New Zealand species as being more closely related to the New Zealand $W$. vernicosa individuals than the Australian $W$. littoricola, though different species are implicated in each case.

In this analysis, $W$. vernicosa is polyphyletic because the sample collected from Raoul Island does not group with the remainder of the samples, which do form a monophyletic group in the chloroplast tree. Due to the uni-parental inheritance of chloroplast DNA the chloroplast tree may not reflect the species tree. Furthermore chloroplast trees have been shown to reflect geographic locations rather than species relationships in a number of cases in the New Zealand flora (e.g. Metrosideros (Gardner et al., 2004)). This does not appear to be the case for Wahlenbergia as the samples that form a monophyletic group are themselves from a range of locations (Chatham Island, Tauranga, Piha and Pakahi Island in the Hauraki Gulf see Appendix 1), which suggests the Raoul Island individual may be a different species altogether. Unfortunately no ITS sequence was generated for the Raoul Island sample identified as $W$. vernicosa, which could have given an independent confirmation of the placement on the chloroplast tree.

Another point to consider is that the two $W$. vernicosa samples that have had their chromosomes counted were from Northland: Surville Cliffs and Three Kings Islands (Petterson et al., 1995). Detailed cytological studies of $W$. vernicosa from across its 
range, including Raoul Island and other Pacific islands, would help to determine whether different chromosome races are present within this species, and even if similar ploidy levels are found, whether these polyploids have originated from different putative parental species (e.g. in Asplenium (Shepherd et al., 2008)). Finally, inclusion of more individuals of this species from throughout its range in the molecular phylogeny would also help determine whether Raoul Island and other Pacific island individuals are conspecific with New Zealand $W$. vernicosa

In summary, the molecular phylogenetic data reported here provide further evidence to support the separation of $W$. vernicosa from the other radicate species of Wahlenbergia in New Zealand, and its recognition as a species in its own right rather than a subspecies of the Australian $W$. littoricola. However, there is no evidence to support the current taxonomy of the other four radicate species. This could indicate that the $W$. gracilis complex should be considered one species, perhaps with varieties to accommodate the morphological variation recorded by Petterson (1997b). Further research using methods able to detect variation at a smaller scale may be able to shed some light on their relationships.

\section{New Zealand rhizomatous species}

The New Zealand species with a rhizomatous growth form (both creeping and suffructose) are recovered as a monophyletic group in all of the datasets (chloroplast, ITS and concatenated) though not for all types of analyses (e.g. the ITS MP analysis does not support a NZ rhizomatous clade). Within this clade the most morphologically distinctive New Zealand species, $W$. cartilaginea (Figure 1.2), is recovered as monophyletic in both the ITS and chloroplast datasets. The specimens of $W$. matthewsii and $W$. congesta subsp. haastii are recovered as monophyletic in the chloroplast data, but not in the nuclear data. These two species are also morphologically distinctive; $W$. matthewsii even has a different growth form (suffructose rhizomatous) that distinguishes it from all other New Zealand Wahlenbergia (Figure 1.1). Unfortunately I was unable to sample any $W$. congesta subsp. congesta individuals to test the status of subspecies in this species. Morphologically the subspecies are very similar, except that $W$. congesta subsp. haastii has smaller capsules and flowers (Petterson, 1997b). There is evidence to 
suggest that individuals of these morphologically diverse species (W. cartilaginea, $W$. matthewsii and $W$. congesta) fall into genetically distinct lineages, which coupled with their morphological differentiation suggests they are appropriately circumscribed at the species level.

Conversely, neither of the other two rhizomatous species, $W$. albomarginata and $W$. pygmaea is monophyletic in either dataset. At the species level there is no DNA sequence evidence to separate $W$. albomarginata from $W$. pygmaea, and as there are few morphological characters that separate them (W. albomarginata has a narrowcampanulate-rotate corolla, whereas in W. pygmaea the corolla is broadly campanulate (Petterson, 1997b)), recognition of these two species may be an artificial split between North and South Island individuals of what is essentially the same species. The lack of any genetic differences separating individuals from the North vs. South Islands could suggest that Cook Strait is not a barrier to gene flow between these individuals, or at least that a periodically emerging land bridge across Cook Strait during the Pleistocene may have facilitated gene-flow (Lewis et al., 1994). If further study indicates that only one species should be recognised, then $W$. albomarginata has priority.

At subspecies rank, in some cases individuals from the same subspecies are grouped together, though this is not evident in subspecies from which individuals were collected across a wider geographic range (e.g. W. albomarginata subsp.

albomarginata) and therefore may be an artefact of the sampling, in that individuals from the same subspecies are often also from the same population due to sampling constraints. Of the four $W$. albomarginata subspecies sampled for this study, subsp. olivina is the only one to form a monophyletic group, and this only occurs in the chloroplast data (two individuals are grouped in the ITS data, the position of the third individual is unresolved). Wahlenbergia albomarginata subsp. olivina is the most morphologically distinctive subspecies, and is able to consistently be identified based on morphological characters (thick white leaf margins, leaves dark green and hairy on top, purplish and glabrous below), and edaphic characters (it only grows on ultramafic rock) (Petterson, 1997b). The evidence of its monophyly, although limited from these analyses, suggests that it is an independent lineage, and that perhaps a subspecies rank is justified in this case. Further exploration may even reveal that an 
elevation to species rank is justified. There is no evidence that subspecies rank is justified for the other subspecies of $W$. albomarginata based on this DNA sequence data, though given the recent evolution of this group it may be unrealistic to expect genetic differentiation among them.

The individual subspecies of $W$. pygmaea are unresolved by the chloroplast data, though the samples from Mt Taranaki (subsp. drucei) are in a separate clade from the individuals from the other two subspecies. In the ITS data the Mt Taranaki samples form a well-supported clade of their own (1.0/86/84) and to a lesser extent so do the samples from the central plateau (W. pygmaea subsp. pygmaea $0.93 / 64 / 64$ ). The samples from the Tararua ranges however are part of a clade also containing $W$. albomarginata subsp. from the north west of the South Island. Although this gives some support for the monophyly of the different subspecies of $W$. pygmaea the conflicting signal from the nuclear and chloroplast datasets makes it difficult to interpret.

\section{Notes on the Australian Wahlenbergia taxonomy}

\section{Australian radicate species}

Although not so extensively sampled as the New Zealand species, some interesting results relating to the Australian Wahlenbergia are presented here. The Australian radicate species do not form a monophyletic clade, though unlike the New Zealand radicates several do form monophyletic species. Species that form monophyletic groups include the two newly described species (discussed further below), $W$. communis and $W$. gracilis (nuclear data only). However, the majority of Australian radicate species sampled for this study do not form monophyletic groups, which could be due to several reasons such as incorrectly identified samples, incorrectly circumscribed species, hybridisation and inappropriate choice of molecular markers (Shaffer and Thomson, 2007). Incorrect identification is a real risk when working with this genus, as the characters distinguishing most of the radicate species are quite precise floral characters, which can be a challenge to apply in the field e.g. ratio of tube to lobe in flowers that are less than one centimetre long. Another factor to consider with the Australian radicate species is that they have mostly been shown to 
contain at least two races of ploidy levels, e.g. the polyphyletic $W$. luteola, which actually morphologically is rather distinctive (opposite linear leaves, bi-colour flowers) has had the chromosomes counted for 10 individuals, three of which had $2 n=18$, and 7 had $2 n=36$ (Smith 1992). It is therefore not unimaginable that the $W$. luteola sample which appears genetically quite different from the other two perhaps has a different ploidy level. Furthermore Smith (1992) reports that hexaploids $(2 n=54)$ hybridise more readily than the diploids $(2 n=18)$ or tetraploids $(2 n=36)$ do. This could potentially explain the peculiar grouping of samples of W. multicaulis (MULT_NSW_Wast), W.planiflora (PLAN_NSW_Nume) and W. littoricola (LITT_NSW_Hart) found in both the nuclear and chloroplast trees (note the ITS dataset is missing the $W$. littoricola (LITT_NSW_Hart) sample), as all of these species have hexaploid counts of $2 n=54$ reported (Smith, 1992).

The relationship between the New Zealand $W$. gracilis complex and the Australian $W$. gracilis species deserves a mention. As touched on in Chapter One the taxonomic history of $W$. gracilis is a confusing one, as there is uncertainty over whether the type of this species (originally Campanula gracilis) is housed at Kew or Gottingen, and whether it is made up of specimens collected in New Zealand or New Caledonia (or both) (Nicolson and Fosberg, 2004). All of the New Zealand radicate species were originally named as C. gracilis (Forster, 1786), hence the use of the term $W$. gracilis complex (Webb and Simpson, 2001), despite the fact that no species in New Zealand have that name now. Petterson (1997a) considered the type to have been collected in New Caledonia, and thought that none of the New Zealand Wahlenbergia were conspecific with the type. However, Smith (1992) considered the Australian entity called $W$. gracilis to belong to this widespread species described by Forster (1786) that also occurred in New Guinea, New Caledonia and New Zealand. In this study the ITS tree contains a moderately supported clade containing all of the New Zealand radicate $W$. gracilis complex individuals (labelled NZ1- in Figure 3.3). This clade has moderate support and does not include $W$. gracilis from Australia. However, the cpDNA tree shows that some cpDNA types are shared among New Zealand $W$. gracilis complex individuals and Australian W. gracilis (clade NZ1+ in Fig 3.4). Further research is required to determine which entities are conspecific, and which belong to the type of $W$. gracilis. Extensive sampling from New Caledonia, New 
Guinea, New Zealand, Australia and Pacific islands will be necessary, analysed using sufficiently variable DNA sequence markers, or AFLPs.

\section{Australian rhizomatous species}

Unlike in New Zealand the three Australian rhizomatous species are not monophyletic, although two of them, $W$. densifolia and $W$. gloriosa, do form a wellsupported clade. The two species form a morphological continuum, with small samples of $W$. gloriosa sharing characters with $W$. densifolia (see descriptions in Smith (1992)). There is evidence in the chloroplast data that $W$. densifolia is differentiated from $W$. gloriosa, but not in the nuclear data. The samples here identified as $W$. densifolia were collected from the same location as one of the $W$. gloriosa samples (start of the Rennix walking track, Mt Kosciuszko see Appendix 1). So the fact that sympatric samples of $W$. gloriosa and $W$. densifolia are genetically differentiated (even only in the chloroplast data) is fairly strong evidence that they do form separate lineages. However, as the $W$. densifolia samples are nested within the $W$. gloriosa samples there may be a case for reducing their level of taxonomic recognition (e.g. to a subspecies of $W$. gloriosa). Further research with greater sampling across both species ranges would be necessary before such a decision could be made. The other rhizomatous species, W. ceracea, forms a clade with most of the $W$. stricta samples and the $W$. telfordii samples (discussed below). There is insufficient resolution to speculate on the evolution of the rhizomatous growth form in Australia.

\section{Newly described species}

Both of the species newly described by Plunkett et al. (2009) represent distinct genetic lineages, a result that is congruent with their recent recognition at the species level. Analysis of DNA sequence data shows that Wahlenbergia telfordii is affiliated with $W$. stricta and the rhizomatous $W$. ceracea, and is in fact indistinguishable from $W$. stricta on chloroplast data alone, but is differentiated by the ITS data. On the other hand using morphological data Plunkett et al. (2009) noted morphological floral and leaf similarities between $W$. rupicola and $W$. stricta, and seed morphological similarities between $W$. rupicola and $W$. ceracea. It is therefore 
surprising that here the $W$. telfordii individuals grouped with $W$. stricta and $W$. ceracea samples, though this had been implicated by the morphological dendrogram in Plunkett et al. (2009). The position of the W. rupicola individuals was less well resolved, as they grouped in a clade containing $W$. littoricola and $W$. communis samples among other species, but were entirely unresolved in the ITS tree. The lack of resolution is not surprising as Plunkett et al. (2009) hypothesised this entity was most closely related to either $W$. glabra (based on an initial morphological assessment), a species not included in this dataset or $W$. luteola (based on the phonetic dendrogram) which in the chloroplast data it does in fact group nearer to than any of the other species included in their morphological assessment.

\section{Summary and conclusions}

The Australasian Wahlenbergia exhibit very low genetic diversity as measured by the chloroplast markers $\operatorname{trn} L-F$ and $\operatorname{trn} K$ and the nuclear ribosomal ITS. The low genetic diversity (especially in the New Zealand species) is probably due to rapid evolution during a period of geological and climatic change, potentially coupled with incomplete lineage sorting and hybridisation. While this low level of variation has resulted in poorly resolved phylogenetic trees a certain amount of information regarding relationships between individuals and species has resulted in an increased understanding of the evolution of the Australasian Wahlenbergia species.

The New Zealand radicate species as a group are probably not monophyletic, though further study is necessary. The only New Zealand radicate species clearly monophyletic in this study is the subtropical radicate $W$. vernicosa, which should be recognised at the species rank and not as a subspecies of $W$. littoricola as it is not closely related to that species. The other radicate species, the $W$. gracilis complex, should be perhaps considered as varieties of a single species, but further detailed studies are needed. The New Zealand rhizomatous species are probably monophyletic, which indicates radiation following a single introduction from Australia. Three morphologically distinctive rhizomatous species have some level of genetic distinction also ( $W$. cartilaginea, $W$. matthewsii and $W$. congesta), which is consistent with their species ranking. The other two species ( $W$. albomarginata and 
W. pygmaea) are indistinguishable on the basis of this study and should perhaps be recognised as one species, though one subspecies of $W$. albomarginata might be a distinct genetic lineage ( $W$. albomarginata subsp. olivina) and requires further study. The Australian species are very poorly resolved, though the two newly described species do form distinct lineages ( $W$. rupicola and $W$. telfordii).

The poorly-resolved and complex phylogenetic relationships exhibited in this study make species delimitation on the basis of sequence data a difficult task. In this study, some species hypotheses have been supported, but a larger number of species hypotheses have not been adequately addressed by this dataset as there are too few characters to distinguish between individuals leading to very poorly resolved trees. In order to address these issues it will be necessary to explore this genus using another method, such as analysis of low-copy nuclear markers or perhaps amplified fragment length polymorphisms (AFLPs). AFLP analysis can be very useful when genetic variability is low, when studying polyploids and when hybridisation is occurring (Meudt and Clarke, 2007), three situations that all apply to the Australasian Wahlenbergia. 


\title{
Chapter Four: Using AFLPs to resolve phylogenetic relationships in a plant
} species complex when nuclear and chloroplast genes fail to reveal sufficient variability

\begin{abstract}
AFLPs were used to analyse 38 individuals of Australasian Wahlenbergia, with a focus on the New Zealand representatives. Overall support values were slightly higher in the AFLP analyses than in the DNA sequence analysis of Chapter Three, indicating greater resolution for this technique. However, the higher support was generally found towards the tips of the branches, whereas deeper relationships were equally poorly supported using both AFLPs and sequence data. The morphologically distinctive $W$. matthewsii and $W$. congesta subsp. congesta were recovered as being distinctively different using AFLP analysis. Members of the $W$. albomarginata/W. pygmaea complex may all belong to the same species, as may the lowland radicate $W$. gracilis complex.
\end{abstract}

\section{Introduction}

An interesting characteristic of the New Zealand flora is its relatively low rate of molecular evolution of commonly used markers such as nuclear ribosomal internal transcribed spacer (nrITS) and chloroplast loci, coupled with high rates of morphological and ecological variation (Winkworth et al., 2005). This pattern is found across several New Zealand plant genera both alpine and lowland (e.g. Myosotis (Winkworth et al., 1999; Winkworth et al., 2002a), Veronica as the hebe complex (Wagstaff et al., 2002; Wagstaff and Garnock-Jones, 1998), Ourisia (Meudt 
and Simpson, 2006) and Sophora (Mitchell and Heenan, 2002)) and contributes to the difficulty in understanding species boundaries and evolutionary relationships in these groups. It also suggests that these species radiations have occurred relatively recently (Winkworth et al., 2005). Indeed molecular clock analyses of nrITS sequence data from several large genera in New Zealand (e.g. Myosotis (Winkworth et al., 2002a), Ourisia (Meudt et al., 2009) and Ranunculus (Lockhart et al., 2001)) suggest that these lineages radiated within the last $5 \mathrm{my}$. The last 5 million years was a period of rapid geological uplift and a changeable climate that created many new and fragmented habitats with fluctuating connectivity (Winkworth et al., 2005), which could well explain the pattern of low molecular vs. high morphological variation. Recent molecular studies of the New Zealand flora indicate this period was also a time of numerous long distance dispersal events between Australia and New Zealand and was therefore an important time for establishing trans-Tasman relationships in the flora (Winkworth et al., 1999).

Nuclear ribosomal DNA (nrDNA) and chloroplast DNA (cpDNA) are commonly used in plant phylogenetic analysis (Alvarez and Wendel, 2003). Chloroplast DNA is favoured because it is easy to extract and amplify due to the large number of copies in each cell (Kelchner, 2000) and universal primers can easily be developed for it (Wolfe and Randle, 2004). Additionally, organelles are usually inherited uniparentally, which potentially results in more straightforward interpretation. Uniparental inheritance restricts the usefulness of cpDNA though, as it reveals only half of the parentage in plants of hybrid or polyploid origin (Small et al., 2004). An additional limitation of cpDNA in molecular phylogenetic studies is its relatively slow rate of evolution, which means that even non-coding cpDNA regions often fail to resolve phylogenies at low taxonomic levels. Nuclear ribosomal DNA (e.g. the internal transcribed spacer (ITS)) has been widely used to complement organellar DNA by obtaining additional, independent, bi-parentally inherited phylogenetic estimates that evolve at a much higher rate. The high level of sequence variation can make alignment very difficult though, and there are a number of other concerns with using ITS as marker (see Alvarez and Wendel (2003) for a good summary). When both cpDNA and ITS sequences fail to resolve phylogenies, the amplified fragment length polymorphism (AFLP) approach has the potential to solve such difficulties (Meudt and Clarke, 2007). 
The low level of variation in DNA sequence data frequently found in New Zealand studies means it can be difficult to resolve phylogenetic trees (e.g. Ourisia (Meudt and Simpson, 2006)). Molecular systematists in New Zealand have started turning to alternative molecular techniques, such as AFLPs, to explore molecular variation in the flora (e.g. Veronica (Meudt and Bayly, 2008), Leocogenes (Smissen and Breitwieser, 2008), Pseudopanax (Perrie and Shepherd, 2009), Metrosideros (Broadhurst et al., 2008), Ourisia (Meudt et al., 2009) and Polystichum (Perrie et al., 2003)). AFLP markers are sampled throughout the (largely nuclear) genome and therefore they can display rare genetic differences in groups with low sequence variation e.g. among closely related species, crop species, or at the intraspecific level (Meudt and Clarke, 2007). This simultaneous analysis of many loci representing the whole genome is also more likely to generate a true species tree, rather than generating a particular gene tree as sequencing does (Després et al., 2003).

The New Zealand species of Wahlenbergia (Campanulaceae) appear typical of New Zealand alpine plant radiations - though half of the species inhabit the lowland - as they are mostly white flowered, polyploid species that occur in a diversity of habitats. Chapter two of this thesis has shown the ancestors of New Zealand Wahlenbergia probably arrived in New Zealand from Australia, then radiated, in the last 5 million years. Chapter three showed the low sequence variation of nrITS and two chloroplast loci and the accompanying difficulties in resolving the relationships of species of Australasian Wahlenbergia because of this. There are two clear lineages present within the New Zealand Wahlenbergia, corresponding to two different growth forms, radicate and rhizomatous. Within the New Zealand radicate group $W$. vernicosa has a unique chromosome count, and there was evidence from chloroplast DNA sequence data that it forms a distinct lineage. However, there was no evidence to falsify the hypothesis that the other four radicate species (the $W$. gracilis complex) are conspecific. Based on cpDNA and nDNA sequence data the New Zealand rhizomatous species are probably monophyletic, which indicates radiation following a single introduction from Australia. Three morphologically distinctive rhizomatous species had some level of genetic distinction based on DNA sequence data ( $W$. cartilaginea, $W$. matthewsii and $W$. congesta), which is consistent with their species ranking. The other two species ( $W$. albomarginata and $W$. pygmaea) were 
indistinguishable on the basis of nuclear and chloroplast sequence data, though one subspecies of $W$. albomarginata formed a distinct group ( $W$. albomarginata subsp. olivina). The New Zealand species of Wahlenbergia are therefore a prime candidate for analysing with AFLP data to attempt to resolve relationships and reconstruct their evolutionary history.

The aim of the present chapter is to analyse AFLP data for Australasian Wahlenbergia to assess species relationships and species limits, with a particular focus on New Zealand species. The current taxonomy and specific research questions of this thesis regarding Australasian Wahlenbergia have been described in detail in Chapter Three. Thus, the monophyly or otherwise of all of the species put forward in Petterson's (1997b) revision will be assessed, with specific interest in whether there is any evidence to falsify the hypothesis that all members of the $W$. gracilis complex are conspecific. The conspecificity of the New Zealand radicate $W$. vernicosa (treated as a subspecies of $W$. littoricola by de Lange and Cameron (1999)) with the Australian $W$. littoricola is of interest too. Evidence for species monophyly will also be assessed in species with the rhizomatous growth form, paying particular attention to the W. albomarginata/W. pygmaea complex and their subspecies. In this research, I follow de Queiroz (2007) in defining species as separately evolving metapopulation lineages. That is, a species is a demographically and genetically interconnected set of populations (metapopulation) that has continuity through time (Mayden, 1997).

Although this definition does not provide an unambiguous cutoff for when speciation has occurred, it emphasizes that the primary goal of species delimitation research is lineage discovery and delimitation by using evidence from numerous sources of data.

\section{Methods}

\section{Sampling and DNA extraction}

Location of samples, collection details and voucher information are presented in Appendix 1. A total of 38 individuals were chosen for AFLP analysis, $31 \mathrm{New}$ Zealand samples and 7 Australian samples. These individuals were selected in order that two or more representatives of each species and subspecies present in New Zealand were included in the dataset. This sampling strategy is effectively that of a 
pilot study i.e. the intention was to assess the level at which the AFLPs are informative for New Zealand Wahlenbergia. Total DNA was extracted from silicadried leaves after manual disruption of dried tissue with a mortar and pestle using a (CTAB) method modified from Doyle \& Doyle (1990) and described fully in Chapter Two. Only DNAs of high quality and high concentration were used. Quality was determined by running out the extracted DNA on a $1.5 \%$ agarose gel and quantity was assessed by use of a nano-drop machine. Only samples with concentrations of DNA greater then $250 \mathrm{ng} / \mu \mathrm{L}$ were used.

\section{Generation of AFLP data}

AFLPs were generated based on the protocol of Vos et al. (1995) using an updated protocol for capillary detection of fluorescently labelled markers (see:

http://awcmee.massey.ac.nz/aflp/AFLP_Protocol.pdf). Total DNAs were digested using EcoRI (Roche) and MseI (NEB) restriction enzymes at $37^{\circ} \mathrm{C}$ for $2 \mathrm{~h}$, followed by 15 mins at $70^{\circ} \mathrm{C}$ to denature the enzymes. If nanodrop readings were higher than $1000 \mathrm{ng} / \mu \mathrm{L}, 3 \mu \mathrm{L}$ of DNA was digested, if less than $1000 \mathrm{ng} / \mu \mathrm{L}, 5 \mu \mathrm{L}$ were digested, thus ensuring a similar amount of DNA in each sample. Complete digestion was checked by running the digests out on a $1.5 \%$ agarose gel. Eco- and Mse-linkers were then ligated to the resulting DNA fragments by incubating with T4 DNA Ligase (Roche) at $37^{\circ} \mathrm{C}$ for 3 hours. Pre-selective PCR amplification was performed using primers $\mathrm{Eco}+\mathrm{A}$ and $\mathrm{Mse}+\mathrm{C}$ in a Biometra $\mathrm{T}$ gradient machine (Whatman, Germany) in a final volume of $20 \mu \mathrm{L}$. Each $20 \mu \mathrm{L}$ volume contained $1 \mu \mathrm{L}$ of ligated DNA, $4 \mu \mathrm{L}$ of $5 \mathrm{M}$ betaine, $2.5 \mu \mathrm{L}$ of $2 \mathrm{nM}$ dNTPs, $2 \mu \mathrm{L}$ of $10 \mathrm{x}$ PCR buffer (Roche), $1 \mu \mathrm{L}$ of each of the primers $(10 \mathrm{pmol} / \mu \mathrm{L}), 0.2 \mu \mathrm{L}$ (corresponding to 1 unit) of Taq DNA polymerase (Roche) and $\mathrm{ddH}_{2} \mathrm{O}$ to make $20 \mu \mathrm{L}$. The PCR protocol consisted of 20 cycles of denaturation at $94^{\circ} \mathrm{C}$ for 30 seconds, annealing at $56^{\circ} \mathrm{C}$ for 1 minute followed by extension at $72^{\circ} \mathrm{C}$ for 1 minute.

Selective PCR amplification was performed using fluorescently-labelled Eco+ANN primer and $\mathrm{Mse}+\mathrm{CNN}$ primer in a final volume of $20 \mu \mathrm{L}$. Each $20 \mu \mathrm{L}$ volume contained $1 \mu \mathrm{L}$ of pre-selective PCR product, $2.5 \mu \mathrm{L}$ of $25 \mathrm{mM} \mathrm{MgCl}_{2}, 2.5 \mu \mathrm{L}$ of $2 \mathrm{nM}$ dNTPs, $2 \mu \mathrm{L}$ of 10x PCR buffer (Roche), $1 \mu \mathrm{L}$ of each of the primers $(10 \mathrm{pmol} / \mu \mathrm{L})$, $0.2 \mu \mathrm{L}$ (corresponding tol unit) of Taq DNA polymerase (Roche) and $\mathrm{ddH}_{2} \mathrm{O}$ to make 
$20 \mu \mathrm{L}$. A step-down PCR program was used. The protocol involved an initial twominute denaturation at $94^{\circ} \mathrm{C}$ followed by ten cycles of $94^{\circ} \mathrm{C}$ for $30 \mathrm{sec}, 30 \mathrm{sec}$ at $65^{\circ} \mathrm{C}-56^{\circ} \mathrm{C}$ (dropping a degree every cycle) and 1 minute at $72^{\circ} \mathrm{C}$ followed by a further 30 cycles with the annealing temperature at $56^{\circ} \mathrm{C}$. Four different fluorescently labelled Eco primers were used and trialled with a selection of potential combinations of eight different Mse $+\mathrm{CNN}$ primers. The following primer combinations were chosen based on a screen involving 4 individuals: 6FAM$\mathrm{Eco}+\mathrm{ACT} / \mathrm{Mse}+\mathrm{CAA}, \mathrm{VIC}-\mathrm{Eco}+\mathrm{ATA} / \mathrm{Mse}+\mathrm{CGT}, \mathrm{NED}-\mathrm{Eco}+\mathrm{ACC} / \mathrm{Mse}+\mathrm{CAC}$ and PET-Eco+AGG/Mse-CAA (hereafter, 6FAM, VIC, NED, PET). All primers were from Sigma except VIC-, NED- and PET- labelled primers (Applied Biosystems). For each individual, selective amplifications of each of the four dyes were mixed together in the ratio of 1:1:1:2 (6FAM:VIC:NED:PET), along with a GS-500 LIZ size standard, and $1 \mu \mathrm{L}$ of each sample was run on an Applied Biosystems Genetic Analyzer (ABI3730) at the Allan Wilson Centre Genome Service (Massey University, Palmerston North, New Zealand). To ascertain reproducibility replicate AFLP profiles were generated from independent restriction digests of the same DNA extraction for six individuals (ca. 10\% of the dataset). Replicates were included in different runs on the 3730 Genetic Analyzer to ensure samples in different runs were comparable.

\section{Parameter optimisation and automated scoring of AFLP data}

Automated scoring was performed on the resulting electronic AFLP profiles using GeneMarker v 1.80 (SoftGenetics). Scoring parameters were optimised following the procedure developed by Holland et al. (2008). After preliminary testing, they determined that peak height threshold (PHT), minimum fragment length (MFL), stutter peak filter (SPF) and local and global detection percentages (LGDP) were the most important parameters to be optimised (Holland et al. 2008). Thirty-six different Wahlenbergia AFLP character matrices were generated that varied by setting PHT to 50,100 or 150 relative fluorescence units (rfu), MFL to 50, 75 or 100 base pairs (bp), SPF to either its default of 5\%, or turned off, and LGDP to its default of $1 \%$ (both local and global) or turned off in GeneMarker. When scoring all matrices the 'smoothing' option was turned on and all other parameters were left at their default 
values in GeneMarker. Data matrices containing characters from all four dyes were exported from GeneMarker and converted into NEXUS format files.

Comparisons of the 36 datasets to determine optimal scoring parameter settings were based on the following measures of accuracy: resolution (and normalised resolution), the number of parsimony informative characters, the number of replicate pairs that were correctly assigned as sister to one another, and the error rates (both Euclidean and Jaccard (Holland et al., 2008)). Holland et al. (2008) also developed a set of Python scripts to streamline the process of analysing the PAUP* output and producing resolution scores, normalised resolution scores and both types of error rates. These scripts were implemented with Barbara Holland's help for this study. Resolution scores were thus calculated by performing 100 repetitions of 100 bootstrap replicates of neighbour joining $(\mathrm{NJ})$ trees created using PAUP* version $4.0 \mathrm{~b} 10$ (Swofford, 2002). For each replicate the bootstrap scores over 50\% were summed and this number was divided by the number of internal edges in each tree, to give a number between $0 \%$ and $100 \%$. Both the number and quality of characters influence resolution scores. To get a measure of character quality independent of sequence length Holland et al. (2008) defined a normalised resolution score, which was calculated by creating datasets the length of the shortest dataset for each combination for parameter settings. The resolution score was then calculated as above. The number of parsimony informative characters and the number of replicate pairs that were correctly assigned as sister to one another were assessed from the PAUP* NJ trees.

Both Euclidean and Jaccard error rates are measures of the replicate error. The Euclidean error rate compares the number of correct calls where a replicate pair both have no peak or both have a peak, to the number of incorrect calls where one half of the replicate pair has a peak and the other half does not. Holland et al. (2008) pointed out that this error rate may be influenced by the size of the data set, as more individuals (especially closely related ones) would increase the number of double absent calls in replicate pairs, thus apparently lowering the error rate. To counteract this Holland et al. (2008) developed an alternative error rate, which doesn't include double absent calls in the denominator, termed the Jaccard error rate. 
To assess the robustness of the phylogenies to changes in parameter settings, majority rule consensus trees were created for each of the 36 datasets. The sets of 36 trees were then analysed using consensus networks as implemented in SplitsTree4 v. 10 (Huson and Bryant, 2006).

The parameter optimisation exercise was performed twice, once on the 36 datasets made up of characters from all four dyes, and once on another 36 datasets with the characters from 6FAM excluded. The 6FAM characters were excluded as further exploration of the samples in GeneMarker revealed that the 6FAM primer combination had failed in some samples, including one replicate. Optimal parameter settings were determined based upon the above analyses by assessing trends in each of the accuracy measures. Following scoring optimization and replicate checking, the six replicate profiles were removed; one additional individual (CART_SI_Hanm1) was also removed as NED failed in that sample.

\section{Phylogenetic analysis}

Phylogenetic trees were reconstructed using maximum parsimony (MP) and Bayesian inference models. MP trees were built using PAUP* v4b10 (Swofford, 2002). MP analysis was performed in a two-step search strategy. First, multiple islands were searched with 10,000 random addition sequence replicates, nchuck $=5$, chuckscore $=1$ and maxtrees $=10,000$. The resulting trees were then swapped to completion with the same settings but chuckscore $=$ no. Support for clades was assessed using 1000 bootstrap replicates, 10 random addition replicates, and MAXTREES $=20,000$ in PAUP*. For Bayesian inference a restriction site (binary) model was used. Although the evolution of AFLP markers is far more complex than the model assumes (Luo et al., 2007), this model has been shown to be useful for analyzing AFLP data nonetheless (Koopman et al., 2008). Four independent chains with 10 million iterations were run using MrBayes v3.1 (Huelsenbeck, 2001). Convergence was assumed to have occurred when the standard deviation of split frequencies was below 0.01, but was also assessed using Tracer v1.4.1(Rambaut and Drummond, 2007). A burn in of 5\% was used. 


\section{Principal coordinate analysis (PCOA) of dominant data}

The program NTSYS-pc (Rohlf, 1990) was used to conduct a principal coordinate analysis $(\mathrm{PCoA})$ to investigate clusters in the dataset. $\mathrm{PCoA}$ is an ordination method, which graphically explores the relationships within the data by mapping a similarity matrix. A PCoA allows the assessment of the dimensionality of the data and a description of the major patterns of variation within and between populations (Ishida et al., 2003). The PCoA was performed using Jaccard distances, DCENTER, EIGEN and MOD3D modules with 3 dimensions analysed and visualised. Initially the full dataset was analysed, followed by subsets of the dataset to further investigate clusters of interest.

\section{Results}

\section{Selection of optimal AFLP automated scoring parameters}

The resolution, normalised resolution, number of parsimony informative characters, the number of replicate pairs that were correctly assigned as sister to one another, and the error rates (both Euclidean and Jaccard) for all 36 datasets are presented in Table 4.1. The number of characters in each of the 36 datasets generated in GeneMarker using different scoring parameter settings varied between 945 and 1473 with 6FAM characters included, and between 714 and 1039 with them excluded. The datasets with the greatest number of characters also had the largest number of parsimony informative characters and the highest resolution scores. Unfortunately they also had the highest error rates and the lowest normalised resolution scores. Nevertheless, overall none of the accuracy measures varied all that much. When 6FAM characters were included the number of parsimony informative characters ranged from 731 to 1204 , replicates correctly paired from 3-5, resolution scores from $56 \%$ - $65 \%$, normalised resolution from $66 \%-71 \%$, Euclidean error rates from $7 \%-9 \%$ and Jaccard error rates from $42 \%-46 \%$. When the 6 FAM characters were excluded the number of parsimony informative characters ranged from 555 to 842, replicates correctly paired from $4-5$, resolution scores from $54 \%-60 \%$, normalised resolution from $80 \%-87 \%$, Euclidean error rates from 7\%-9\% and Jaccard error rates from $42 \%-46 \%$. 
When other published AFLP studies have reported Euclidean error rates, they are usually between $2-5 \%$ (see (Bonin et al., 2004)). The higher error rate reported here could be due to the low number of correctly paired replicates (see Table 4.1). When the 6FAM characters were excluded the error rates decreased only slightly for some datasets, despite the higher replicate pairing rate. The higher pairing rate was due to the consistent pairing of the $W$. vernicosa (VERN_NI_Piha) pair, as 6FAM had failed for one of the replicates. The two replicates that mostly did not pair even when 6FAM characters were excluded were $W$. akaroa (AKAR_SI_Bank1) and $W$. matthewsii (MATT_SI_Mar12). In all 36 datasets the W. akaroa replicates grouped in a clade containing only the two replicates and the other $W$. akaroa (AKAR_SI_Bank2) sample (data not shown), which indicates merely that the AFLPs are unable to distinguish between individuals from the same population. In contrast there was no pattern to the relationships between the $W$. matthewsii replicates in the first 24 datasets, they were often in different clades (though the clades always had low bootstrap support). For the remaining datasets they followed the same pattern as the $W$. akaroa replicates.

As well as the low replicate pairing, the higher Euclidean error rates reported in this study could be due to the completely automated scoring process implemented here. Most published AFLP studies have used a combination of manual or semi-automated scoring. Holland et al. (2008) reported similarly high Euclidean error rates in their fully automatedly scored datasets, and after exploring their results with ReplicateError (available from http://baseplugins.thep.lu.se/wiki/se.lu.onk.ReplicateError) suggested the majority of errors were scoring errors (rather than PCR errors). They therefore suggested that the incorporation of improved scoring algorithms into GeneMarker would further increase its power and usefulness (Holland et al., 2008). They also nevertheless advocated use of automated scoring of AFLPs, as manual scoring is subjective, timeconsuming and not repeatable in the same way that automated scoring is (Holland et al., 2008). 


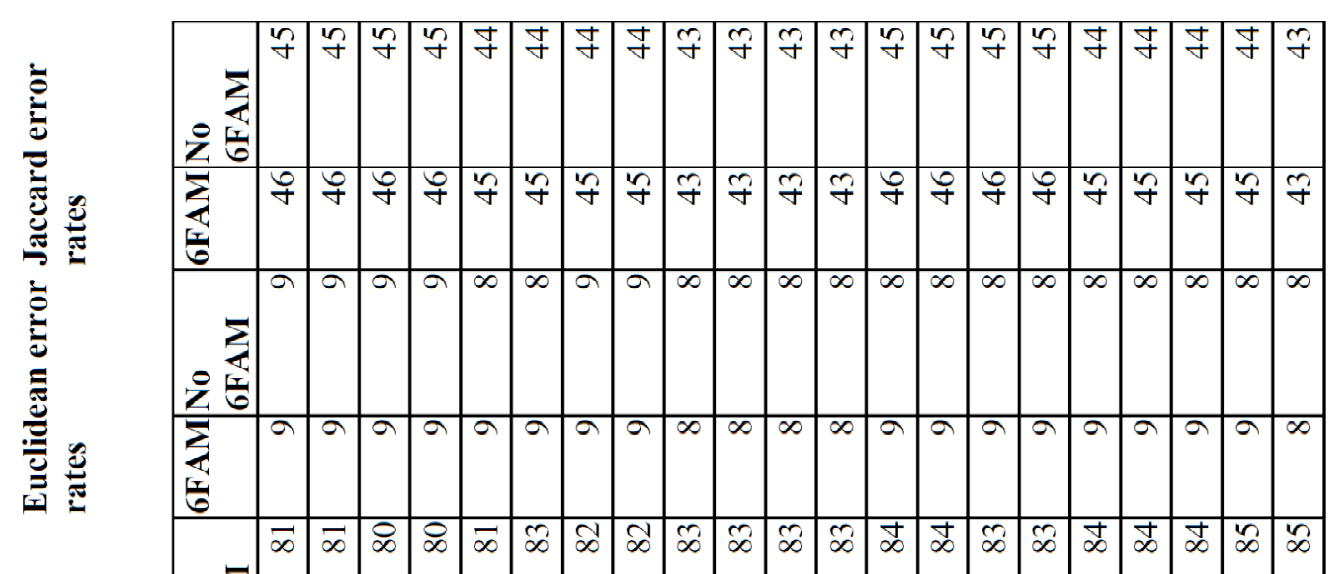

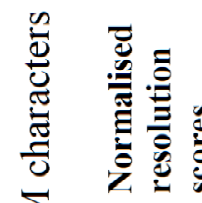

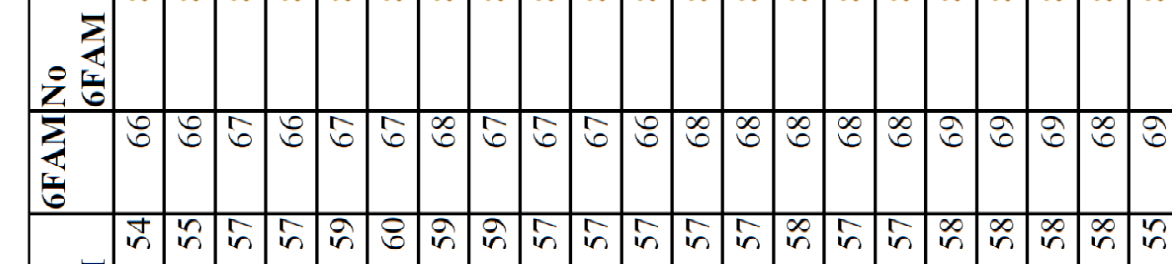

造

$\stackrel{8}{\approx}$

.

三

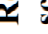

党:

흔

능

80

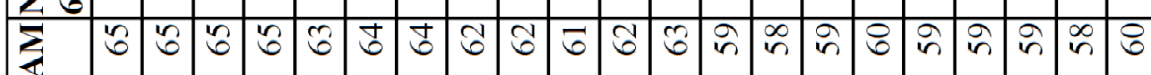

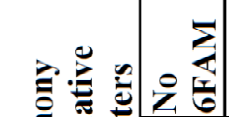

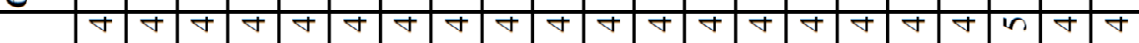

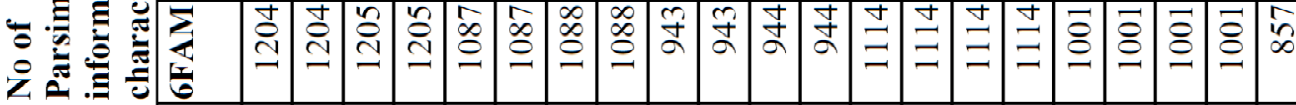

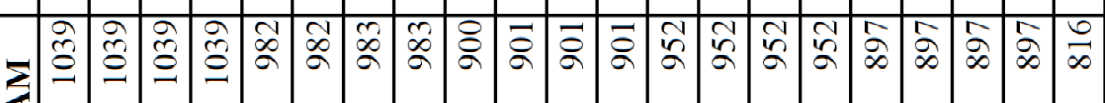

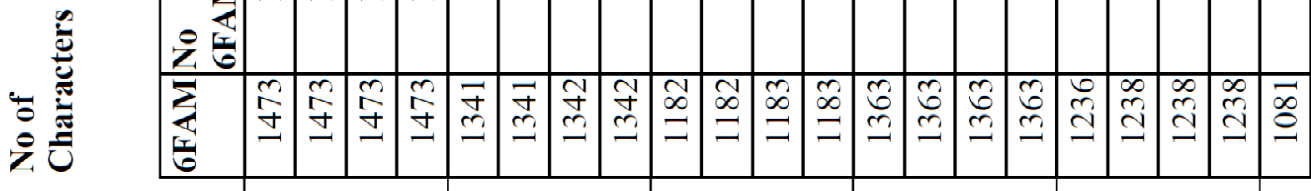

× @

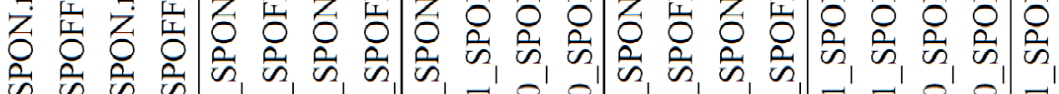
تُ تُ

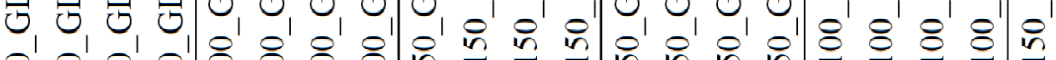

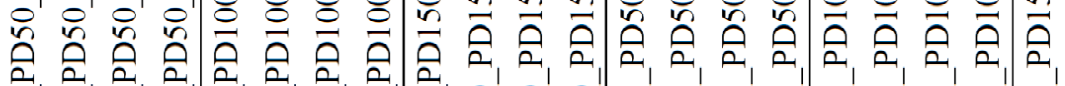

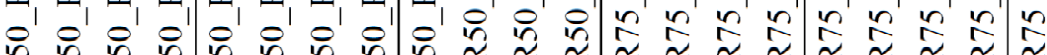

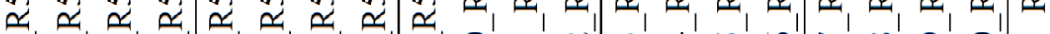

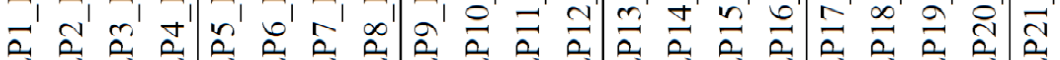

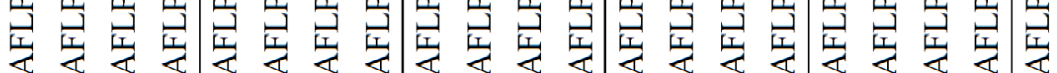

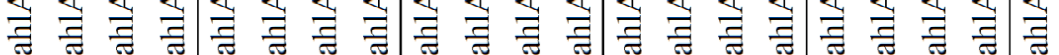

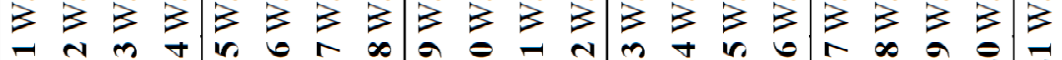




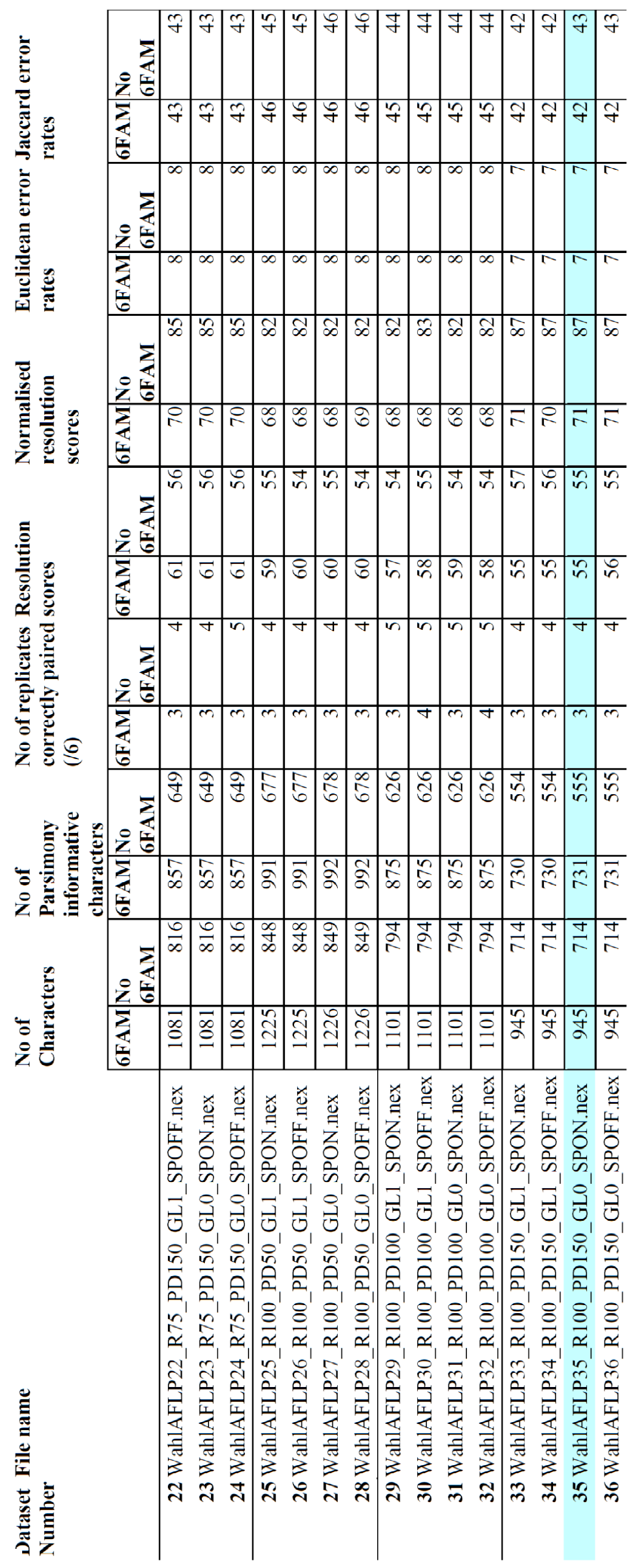




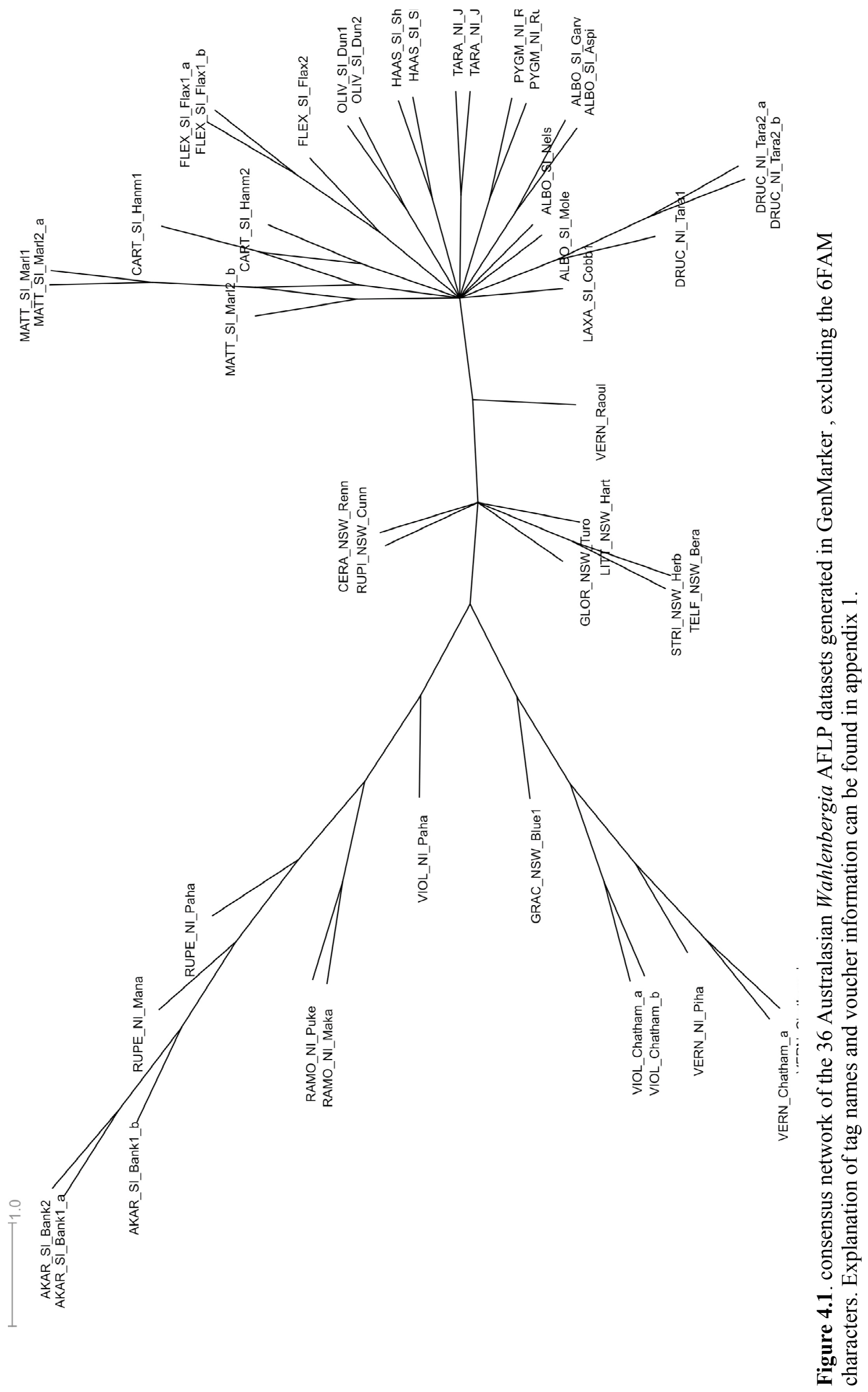


The small amount of variation in all measures suggests this particular dataset is relatively robust to changes in the scoring parameters, which is also indicated by the lack of "boxiness" in the majority rule consensus network (Figure 4.1). The main conflict is in the placement of the $W$. matthewsii replicates. The low variation suggests it is of low importance which dataset is selected for use in this instance. Therefore, I decided to treat the correct replicate pairing as the most important indicator of which datasets to use, which meant excluding 6FAM characters. Of the datasets with 6FAM characters excluded, datasets 25-36 were better at placing the $W$. matthewsii replicates. Within these datasets I selected the dataset with the lowest error rates and highest normalised resolution score. The optimal scoring parameters were selected to be PHT of 150, MFL of 100, SPF on and LGDP off (dataset number 35 , highlighted in blue in Table 4.1). With 6FAM characters included this set of scoring parameters yielded the highest normalised resolution score $(71 \%)$ and the lowest error rates (Euclidean $=7$, Jaccard $=42$ ). With 6FAM excluded this set of scoring parameters again had the highest normalised resolution score $(87 \%)$, and the lowest Euclidean error rate (7), and the Jaccard error rate was second lowest (43, rather than 42).

\section{Phylogeny of New Zealand Wahlenbergia based on AFLP data}

The parsimony analysis returned five most parsimonious trees, one of which is shown here with bootstrap values added (Figure 4.2). The Bayesian analysis ran for 10 million generations, at which point the standard deviation of split frequencies was 0.003. A 50\% majority rule consensus tree was created (Figure 4.3). The Bayesian and MP trees presented here are not rooted, as it is unclear which species should be considered the root of the tree based on the DNA sequence phylogenies reconstructed in chapter three. The 50\% majority rule trees reconstructed with AFLP data using Bayesian and MP estimation had similar topologies, though with some important differences (note the 50\% majority rule MP tree is not shown, but instead bootstrap values of greater than $50 \%$ are recorded on one of the most parsimonious trees Figure 4.2). Overall, the Bayesian tree had higher posterior probability (pp) values than the parsimony tree bootstrap (bs) values, which is in line with previous 


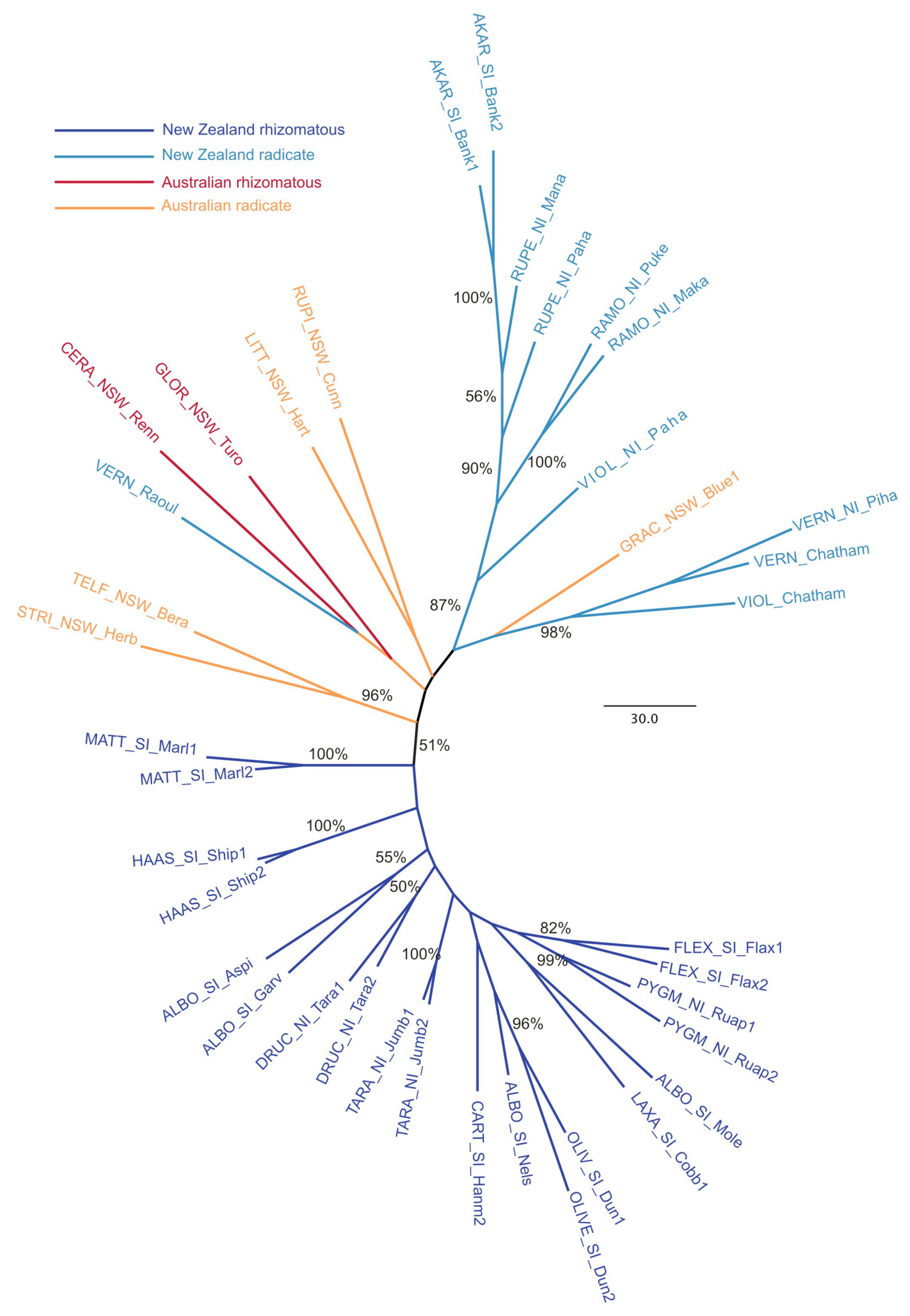

Figure 4.2. One of the five most parsimonious trees reconstructed from Australasian Wahlenbergia AFLP data. Numbers next to branches are bootstrap values (\%). Explanation of tag names and voucher information can be found in Appendix 1. 


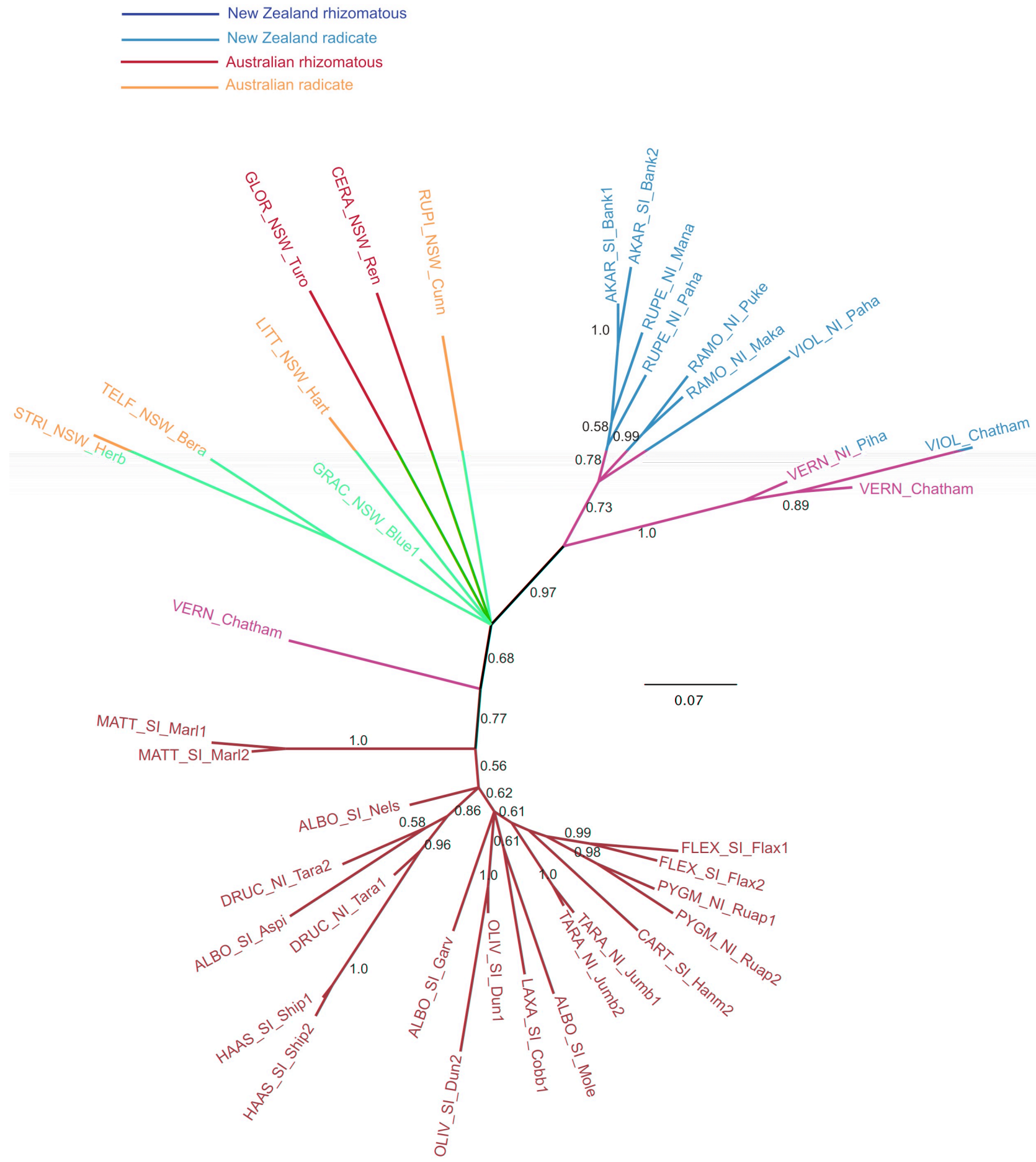

Figure 4.3 Bayesian 50\% majority rule consensus tree of Australasian Wahlenbergia based on the AFLP dataset. Numbers near branches are posterior probability (pp) values. See Appendix 1 for an explanation of the tag names and voucher information. 
studies (Erixon et al., 2003; Wilcox et al., 2002), and can be explained by the fact that posterior probabilities and parsimony bootstrap support values measure different characteristics of the data (Alfaro et al., 2003). Posterior probabilities measure the probability of a node being correct, conditional on the evolutionary model employed and the data set. Therefore their reliability heavily depends on the accuracy of both the data sample and the evolutionary model, which has been acknowledged as being lacking in the case of analysis of AFLP data (Luo et al., 2007). Parsimony bootstrap values are different in that they measure the sensitivity of the observed results to the sampling error associated with collecting characters from a hypothesized underlying character distribution, without the use of an explicitly specified evolutionary model (Alfaro et al., 2003). Given the fact that posterior probabilities and parsimony bootstrapping measure different features of the data, the most reliable nodes are those that have high values of both.

The Bayesian and MP analysis methods both grouped the New Zealand rhizomatous species into a poorly supported clade $(0.77$ posterior probability $(\mathrm{pp}), 51 \%$ bootstrap support (bs)). The Bayesian 50\% majority rule tree (Figure 4.3) recovered the New Zealand radicate species (excluding the $W$. vernicosa sample from Raoul Island (VERN_Raoul)) in a clade with high (0.97) pp, but this relationship was unsupported using parsimony methods (Figure 4.2). In both trees the position of the Australian samples is unresolved between the New Zealand rhizomatous and New Zealand radicate groups. Higher support values were found usually on branches connecting terminal taxa, which corroborates the general notion that AFLP markers are most reliable at the lower taxonomic levels (Mueller and Wolfenbarger, 1999).

Within the New Zealand radicates, $W$. akaroa $(1.0 \mathrm{pp}, 100 \% \mathrm{bs})$ and $W$. ramosa ( $0.99 \mathrm{pp}, 100 \% \mathrm{bs}$ ) formed clades in both analyses while $W$. violacea and $W$. rupestris did not. Interestingly the $W$. vernicosa samples (excluding VERN_Raoul) formed a monophyletic group in the parsimony analysis $(<50 \% \mathrm{bs})$, but in the Bayesian analysis the $W$. vernicosa sample from the Chatham Islands (VERN_Chatham) formed a clade with the $W$. violacea (VIOL_Chatham) sample also from the Chatham Islands (0.89 pp). The position of the Australian $W$. littoricola (LITT_NSW_Hart) sample was unresolved, but it was not within the clade containing the New Zealand $W$. vernicosa samples. 
Within the New Zealand rhizomatous clade samples of $W$. matthewsii (1.0 pp, $100 \% \mathrm{bs})$ and $W$. congesta $(1.0 \mathrm{pp}, 100 \% \mathrm{bs})$ formed species clades, but the $W$. albomarginata and W. pygmaea samples did not. However, subspecies of these two alpine species mostly formed clades, apart from the $W$. albomarginata subsp. albomarginata individuals that were spread throughout the rhizomatous clade in both analyses, and the $W$. pygmaea subsp. drucei samples that grouped in separate clades in the Bayesian tree.

\section{Cluster analysis using principal coordinates analysis}

Principal coordinates analysis revealed similar patterns to the tree building methods (Figure 4.4); the NZ rhizomatous species were clearly separated from the remaining species. The New Zealand radicate species also formed a separate cluster with the exception of the $W$. vernicosa sample from Raoul Island (VERN_Raoul), which was closer to the Australian species. The Australian samples cluster tightly together and are separate from the New Zealand samples. Dimension one explained $9.4 \%$ of the variation, dimension two $5.3 \%$ and dimension three $4.8 \%$. The New Zealand radicate samples are fairly well separated on dimensions $1 \& 2$ (Figure 4.4) and the NZ rhizomatous species are mainly separated on dimensions $1 \& 3$, particularly the individuals of $W$. matthewsii and $W$. congesta subsp. haastii. Individuals of Wahlenbergia albomarginata, W. pygmaea and W. cartilaginea are not well separated from each other or as species. A reduced dataset containing only members of $W$. albomarginata and W. pygmaea was analysed (Figure 4.5). With the other samples removed, more structure is visible, and members of the same subspecies generally group together. However, there is still no clear separation between $W$. albomarginata and $W$. pygmaea. Of this reduced dataset dimension one explained $10.5 \%$ of the variation, dimension two $9.4 \%$ and dimension three $8.6 \%$.

\section{Discussion}

AFLPs are a useful technique to explore genetic variation within New Zealand Wahlenbergia based on the tree-like phylogenies recovered using MP and Bayesian techniques, and the distinct clusters formed using PCoA. Given the low posterior 


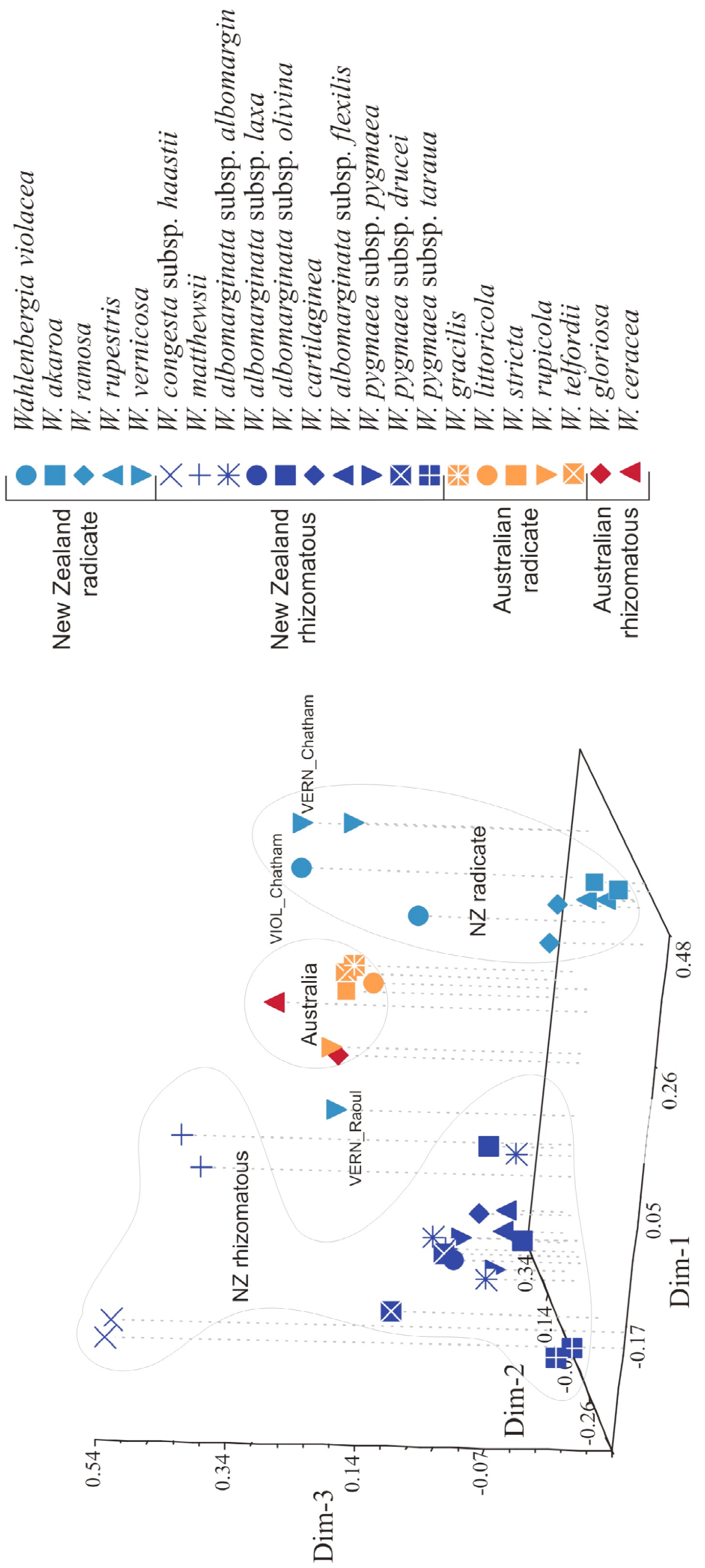

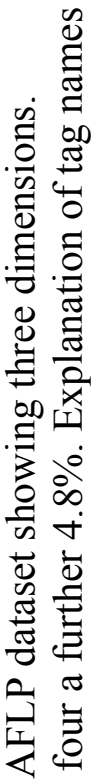

.

จ

ฐ.

ह

ह

.్ํำ ㅇํㄹ

的

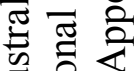

过:

谟

ชิ สี 두

ن용

品

츌

क ज्ञ

政

当

8 प

은

웅

槥

+ 。 ํㅠ

勿

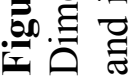




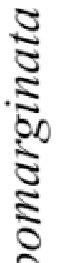

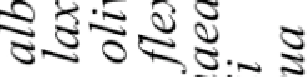

की

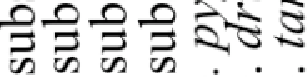

i $\approx$ के

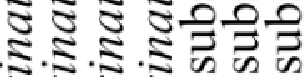

200000000

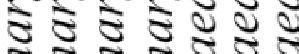

ㅇำ

హ)

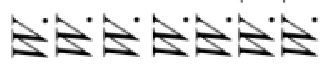

米・쇼

ฮี

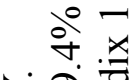

$\therefore \frac{2}{0}$

छี Оิ

on:

政

फ $\approx$

¿

䒕

ㄴ. जै

$\overrightarrow{0}$ 过

0 .

की in के

20

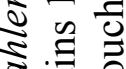

$\frac{\pi}{2}$

₹

政

접 플

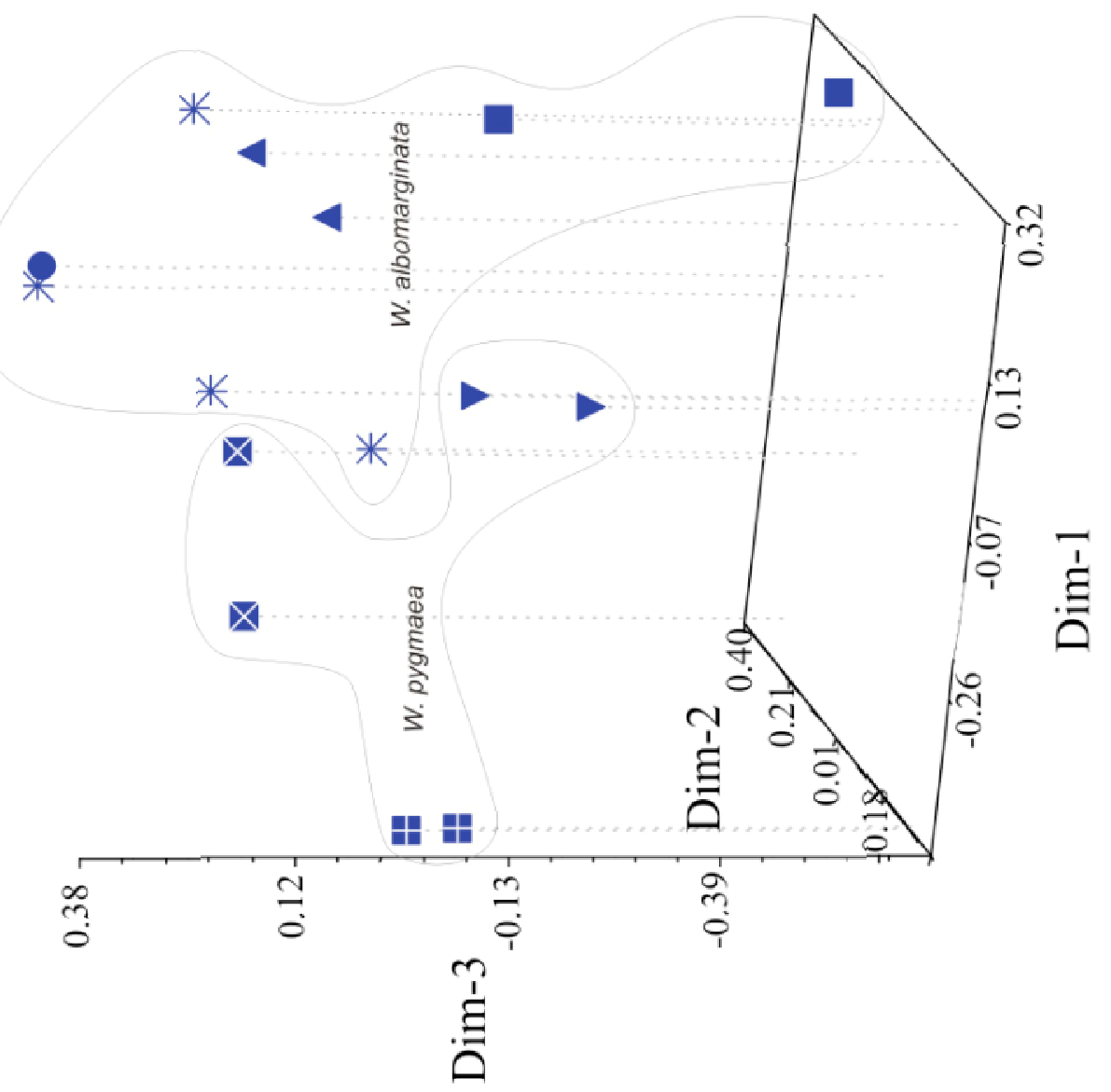

$3 \cdot \frac{0}{0}$

ब..

芑

$\ddot{\theta} \dot{0}$

후을

पू

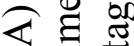

记

¿

을 을

$\lambda . \Xi$

를 远

\&

氜

$\exists$ 艾

8 :

०े है

용

ป

ก.

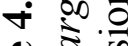

ป ई

일 : 
probabilities and bootstrap support for branches deeper in the trees it seems likely that AFLPs will be more useful at species delimitation rather than assessing phylogenetic relationships among species. Other studies on New Zealand plants using AFLP data have come to a similar conclusion (e.g. Ourisia (Meudt et al., 2009)) and this is consistent with the accepted view that AFLPs are more useful at lower taxonomic levels (Meudt and Clarke, 2007; Mueller and Wolfenbarger, 1999). Unfortunately, this means we have not yet managed to discover a molecular technique that is able to resolve deeper relationships within the Australasian Wahlenbergia. Nevertheless, AFLPs will be a useful technique for exploring species boundaries within ambiguous species complexes in this genus.

\section{Phylogenetic patterns and species delimitation in New Zealand Wahlenbergia based on AFLP}

\section{Radicate species}

The New Zealand radicate species (excluding $W$. vernicosa from Raoul Island) form a cluster in the PCoA analysis and a well-supported group in the Bayesian tree analysis $(0.97 \mathrm{pp})$. They do not form a clade in the MP tree, where $W$. gracilis (an Australian radicate species) is nested within them in an unsupported clade which is unable to be clearly separated from the remaining Australian samples (Figure 4.2). This repeats the pattern found in Chapter Three of this thesis using nuclear and chloroplast DNA sequences, where there was also no clear separation of Australian and New Zealand radicate samples. From the dating analyses conducted in Chapter Two the radiation of all of the Australasian Wahlenbergia was estimated to have occurred about 3.7 mya, and it appeared the New Zealand radicate species probably radiated more recently still about 0.5 mya. This may have been insufficient time for reciprocal species monophyly to have evolved (Shaffer and Thomson, 2007) and therefore the lack of clear structure in the radicate group may simply reflect recent evolution.

Despite this recent evolution, there is some structure within the New Zealand radicates; the $W$. akaroa samples form a well-supported clade (1.0 pp, $100 \% \mathrm{bs})$, as do the $W$. ramosa samples ( $0.99 \mathrm{pp}, 100 \% \mathrm{bs})$. These also appear to be closely 
related based on the PCoA analysis. Neither of these species formed monophyletic groups in the analysis of DNA sequence data. That the relationships are recovered using AFLP data could indicate a greater resolution found using this method. Alternatively it could reflect the smaller sampling in this AFLP study, as two $W$. ramosa samples that grouped separately to the other New Zealand radicate sample in the nuclear and chloroplast sequence trees were not included in the AFLP dataset. However, because the two $W$. akaroa individuals that were included in all studies received high support in the AFLP analysis this suggests more information is available using AFLP data relative to DNA sequence data.

The other NZ radicate species do not form monophyletic groups in the AFLP trees. This is not surprising for $W$. rupestris and $W$. violacea as they do not form monophyletic groups in the DNA sequence data either, but the $W$. vernicosa samples (excluding the one collected from Raoul Island) were monophyletic at least in the chloroplast tree. In the parsimony AFLP analysis the clade containing the two $W$. vernicosa samples (one of which, VERN_Chatham, is from the Chatham Islands) also includes a $W$. violacea sample that was collected from Chatham Islands (VIOL_Chatham). Samples of $W$. vernicosa that have had their chromosomes counted are tetraploid $(2 n=54)$, whereas $W$. violacea (and all other New Zealand temperate radicate species) are octoploid ( $2 n=72)$ (Petterson et al., 1995). Albach (2007) found that AFLPs are sensitive to the analytical method employed (i.e. different relationships are recovered), especially if hybridisation and allopolyploidy are involved. The origins of the New Zealand polyploid Wahlenbergia species are unknown, but analysis of samples with different ploidy levels could explain why the relationship between the $W$. violacea and $W$. vernicosa samples changes subtly with different analysis methods (parsimony vs. Bayesian). Detailed genomic and hybridisation studies would be needed to test this hypothesis. Despite the nonmonophyly of $W$. vernicosa in both the AFLP and nuclear DNA sequence data the consistent morphological differences (e.g. glossy leaves) and different chromosome number appear to set it apart from the other New Zealand radicate species.

The position of the Raoul Island sample identified as W. vernicosa (VERN_Raoul) is again unresolved, though the PCoA analysis appears to group it more closely with the Australian samples than the other New Zealand samples. The single Australian 
W. littoricola (LITT_NSW_Hart) sample included in the AFLP dataset is more closely related to other Australian species than it is to the $W$. vernicosa samples, a pattern also found in chloroplast and nuclear sequence data. The patterns observed in the nrDNA, cpDNA and AFLP are not those expected from conspecific samples, thus they falsify the hypothesis (de Lange and Cameron, 1999) that these entities are conspecific.

Overall this dataset yields little evidence for speciation within the $W$. gracilis complex (W. violacea, W. akaroa, W. ramosa and $W$. rupestris) despite the monophyly of $W$. akaroa and $W$. ramosa. The two $W$. akaroa samples were from the same population, and both of the $W$. ramosa samples were from the Wellington west coast, suggesting the AFLP could be finding population level geographic structure within a single species, rather than species level variation. Additionally the morphological characters differentiating $W$. ramosa and $W$. akaroa are no more than those differentiating the non-monophyletic $W$. violacea and $W$. rupestris. It appears that $W$. akaroa is derived from within the $W$. rupestris samples with high support (Figures 4.2 and 4.3). This result ties in with the original species diagnosis in Petterson (1997b), which described W. akaroa as being "similar to W. rupestris, differing in the pale-violet corolla..." Although these findings do not refute a hypothesis that the $W$. gracilis complex is a single variable species, the flower size differences (Petterson, 1997b) indicate that more sampling and further independent tests should be conducted.

\section{Rhizomatous species}

The New Zealand rhizomatous species form a poorly supported clade in both the parsimony and Bayesian tree building methods $(0.77 \mathrm{pp}, 51 \% \mathrm{bs})$. This clade was also recovered using DNA sequence data (Chapter Three), with slightly lower support values (Figures 3.3-3.5). Within this clade $W$. matthewsii and $W$. congesta subsp. haastii form distinct lineages in the genus based on the AFLP data. This is recovered by both the parsimony and Bayesian tree building methods, as well as the PCoA. There was also some evidence from the DNA sequence data that these species were distinct from each other and the remaining species, and morphological characters also suggest these represent distinct genetic lineages that should continue 
to be treated at the species rank. Only one specimen in the AFLP analyses represented the other morphologically distinct rhizomatous Wahlenbergia, $W$. cartilaginea (CART_SI_Hanm2). Despite its very different morphology this one specimen clusters tightly with the $W$. albomarginata/W. pygmaea complex in the PCoA analysis and its placement is unresolved in both tree building methods for the AFLP data. However, its distinct morphology and habitat (it is a scree plant), and monophyly in phylogenies reconstructed from both nrDNA and cpDNA sequence data, is sufficient evidence to falsify a hypothesis of conspecificity with the other New Zealand rhizomatous species.

As found in the DNA sequence data the two species $W$. albomarginata and $W$. pygmaea do not form monophyletic groups. The samples from these two species were analysed using PCoA without the rest of the samples to try to tease out their relationships. This analysis, along with the tree building methods, showed that despite the non-monophyly of the two species several of their subspecies do form groups or clades. As discussed in Chapter Three this could partly be an artefact of the reduced range of some of the subspecies in that the duplicate samples are from the same population whereas samples of the more widespread (non-monophyletic) subspecies e.g. W. albomarginata subsp. albomarginata are from a range of populations. This AFLP study could not refute the hypothesis that $W$. albomarginata and $W$. pygmaea are conspecific. This question deserves further study and AFLPs are potentially useful to test this with increased sampling to represent different populations of all subspecies from throughout their ranges.

\section{Trans-Tasman relationships}

AFLP data are not able to distinguish among the Australian samples, which is probably due to inadequate sampling as only one individual of each of seven species was included.

\section{Summary and conclusions}

Similar patterns of relationships were found with this analysis of AFLP data relative to those found using analyses of chloroplast and nuclear DNA. Overall, support 
values - which can be used as a proxy for level of resolution within the data (Holland et al., 2008) - were slightly higher in the AFLP analyses. The support values were generally higher towards the tips of the branches, whereas deeper relationships were equally poorly supported using both AFLP and sequence data, suggesting neither method has the appropriate level of variation to elucidate these relationships. It may be possible to find a more variable DNA marker (such as low copy nuclear genes), which may be able to provide information at this level. Although several low-copy nuclear markers trialled in chapter three could not be successfully sequenced, others should be attempted. It is also possible that there are no molecular techniques able to resolve the relationships in question, as there may not be the appropriate signal in the genomes. These kinds of molecular techniques rely on mutations that arise by chance, and the probability that mutations will have occurred that are able to correctly assign each individual to a species group must be relatively low, as their radiation (in Australasia) has probably occurred only in the last $3.7 \mathrm{my}$. Thus, some level of fuzziness and uncertainty in delimiting young species at the beginning of differentiation as shown here is therefore perhaps not unexpected (Shaffer and Thompson, 2007). Hence, rapid evolution and species radiation, such as that occurring within Australasian Wahlenbergia, continues to present a challenge to systematists and taxonomists. 


\section{Chapter Five: General Discussion}

The overall aim of this thesis was to explore the evolution of the Australasian Wahlenbergia at three different scales. At the large scale I undertook a molecular phylogenetic analysis exploring the biogeography of Wahlenbergia including samples from South Africa, Europe, Australia and New Zealand. At the medium scale I assessed the current taxonomy of the New Zealand Wahlenbergia (and Australian species where sampling permitted) using DNA sequencing, and at the small scale I further explored relationships among New Zealand Wahlenbergia species using AFLP techniques.

\section{Large scale phylogeny and biogeography}

At the large scale, the genus Wahlenbergia, which comprises $\sim 260$ species worldwide, was confirmed to be polyphyletic in a phylogeny reconstructed using $\operatorname{trnL}-F$ and ITS sequence data from $\sim 20 \%$ of the species in the genus, although the majority of Wahlenbergia species formed a clade. Further sampling is required to confirm whether this monophyly extends to include Asian and Pacific Wahlenbergia, and whether it is maintained when other closely related wahlenbergioid species are included in the dataset. The genus originally evolved in South Africa (with a herbaceous growth form) and began diversifying about 16.2 mya before dispersing to Australasia about 3.7 mya and radiating into 45 species and subspecies there. It is unclear whether this dispersal event included a step-wise progression through Asia, as unfortunately no Asian Wahlenbergia were included in this study. Two introductions from Australia to New Zealand appear likely, leading to two radiations: one of species with the radicate growth form and one of species with the rhizomatous (both creeping and suffructose) growth form. The ancestor of the New Zealand rhizomatous species arrived and began diverging about 1 mya, and the ancestor of the radicate species about 0.5 mya. The recent evolution of the Wahlenbergia genus as a whole, and the relatively recent introductions to New Zealand, refute the 
Gondwanan vicariance hypothesis for the origins of the New Zealand Wahlenbergia and instead provide overwhelming evidence for arrival via long distance dispersal. Recent studies have shown long-distance dispersal to be a common component in forming the New Zealand flora e.g. Plantago (Tay et al., 2010), Veronica (as the hebe complex) (Wagstaff and Garnock-Jones, 1998) and Pittosporum (Gemmill et al., 2002).

\section{Medium and small scale: Relationships between species}

\section{and taxonomy}

The Australasian Wahlenbergia exhibit very low genetic diversity as measured by the chloroplast DNA markers $\operatorname{trn} L-F$ and $\operatorname{trn} K$ and the nuclear ribosomal DNA marker. The low genetic diversity (especially in the New Zealand species) is probably due to rapid evolution during a period of geological and climatic change, perhaps coupled with incomplete lineage sorting and hybridisation (Winkworth et al., 1999). While this low level of variation has resulted in poorly resolved phylogenetic trees a certain amount of information regarding relationships among individuals and species has resulted in an increased understanding of the evolution of the Australasian Wahlenbergia species.

With greater sampling in Chapter Three, the New Zealand radicate species no longer formed a clade as they had in Chapter Two, although further study is necessary as this could simply be due to the lack of variation in the markers used in this chapter, and does not necessarily point to ongoing gene-flow between Australia and New Zealand Wahlenbergia. Other studies on the Australasian flora have also found very low levels of sequence divergence between Australia and New Zealand (e.g. Craspedia (Ford et al., 2007), Sophora (Hurr et al., 1999; Mitchell and Heenan, 2002) and Veronica (Wagstaff and Garnock-Jones, 1998). Although the relationship was unsupported, the Australian and New Zealand radicate species did appear to fall into two separate groups when analysed with the more sensitive AFLP data for instance. 
The monophyly of the New Zealand rhizomatous species was confirmed using DNA sequence and AFLP data, although as in Chapter Two, their relationship with other Wahlenbergia species remained unresolved. Interestingly there did not appear to be a close relationship between the Australian rhizomatous species included in this study and the New Zealand rhizomatous clade as was hypothesised by Smith (1992). Unfortunately this study did not include any samples of the Tasmanian rhizomatous $W$. saxicola, which was even considered conspecific with the New Zealand rhizomatous species at one time, as this may represent the sister species to the New Zealand rhizomatous clade. Close relationships between Tasmanian and New Zealand species has been found in other plant groups e.g. Pachycladon (Heenan et al., 2002).

The Australian Wahlenbergia do not form a monophyletic group in any of the DNA sequence analyses, but they do form a cluster in the AFLP analyses. This lack of monophyly perhaps is not surprising given the ancestors of the New Zealand Wahlenbergia arrived from Australia only in the last ca. 1 my, meaning reciprocal monophyly may not have had sufficient time to evolve (Shaffer and Thomson, 2007). Interestingly, neither the Australian rhizomatous nor the Australian radicate species form monophyletic groups. Smith (1992) had hypothesised that all of the Australian rhizomatous species were closely related (termed the $W$. gloriosa group), and also split the radicate species into three other groups that he hypothesised as being related to each other, termed the $W$. scopulicola, $W$. gracilenta and $W$. communis groups. Within the $W$. communis group he further identified sub-groups termed the $W$. graniticola, $W$. planiflora and $W$. gracilis groups. This study included no species belonging to the $W$. scopulicola group, and only one belonging to the $W$. gracilenta group ( $W$. preissii) so monophyly of these groups was not able to be assessed. However, the $W$. preissii sample had an identical chloroplast haplotype to Australian radicates belonging to the $W$. communis and $W$. gracilis groups, as well as several New Zealand radicate samples (e.g. see placement in Figure 3.5), which indicates there is little evidence to support this hypothesised group. None of the other groups proposed by Smith (1992) were reconstructed as being monophyletic in this thesis either. However, sampling of the Australian species was not extensive in this thesis, and further phylogenetic research is warranted. 


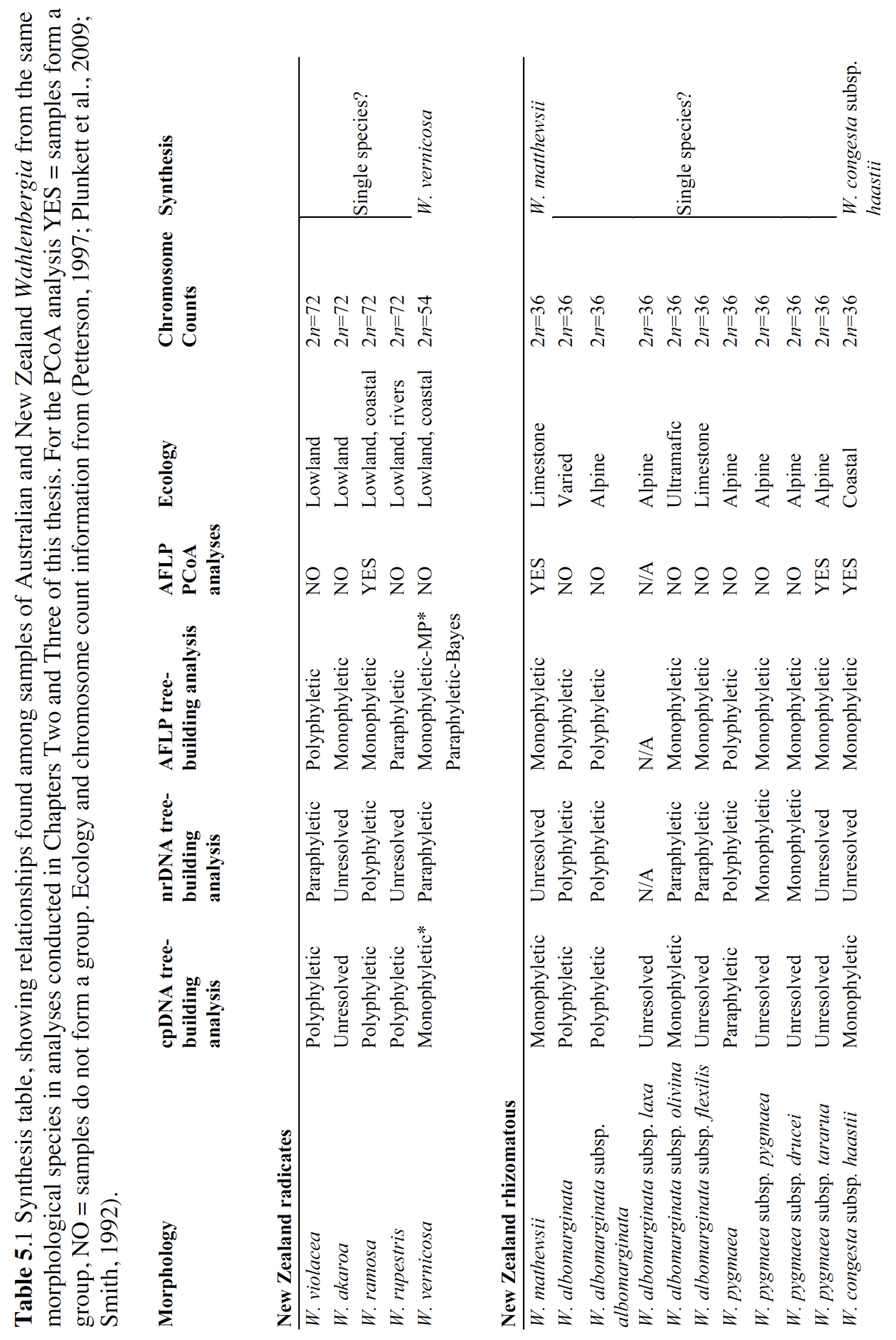




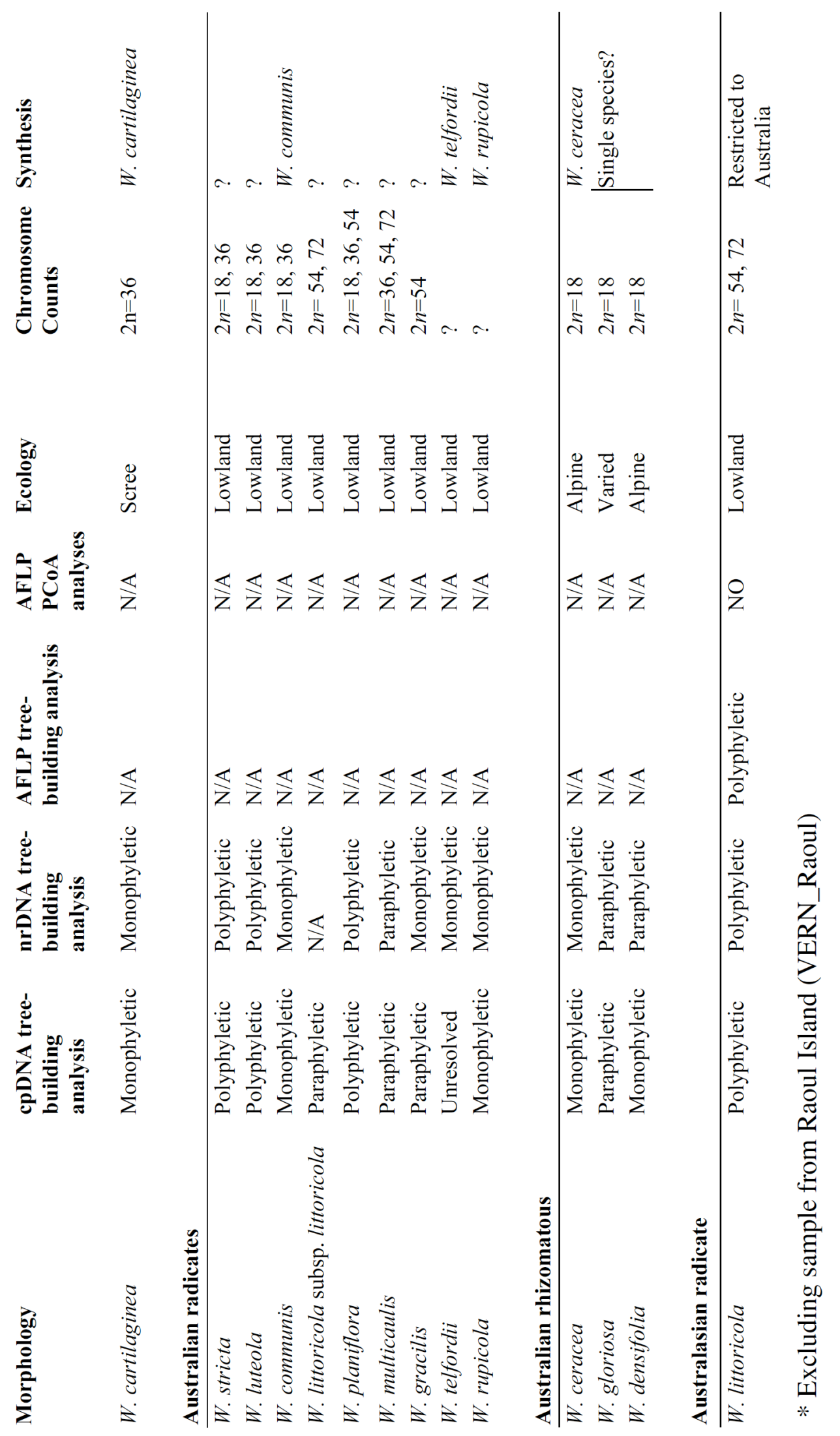


Although phylogenetic relationships among Wahlenbergia species are poorly resolved and difficult to interpret in this thesis, some relationships at the species level could be clarified. Other studies on the New Zealand flora have found that molecular data (both DNA sequences and AFLP) are useful at the same taxonomic level (e.g. Ourisia (Meudt et al., 2009). The complex phylogenetic relationships exhibited in this study make species delimitation on the basis of sequence data a difficult task. In this case some species hypotheses have been supported, but a larger number of species hypotheses have not been adequately addressed by this dataset as there are too few characters to distinguish between individuals leading to very poorly resolved trees. The unified species concept was adopted in this study, with the central line of investigation involving monophyly at one or multiple DNA loci and AFLP analysis, coupled with morphological, chromosomal and/or ecological distinctness. Table 5.1 displays all of the Wahlenbergia species represented by more than one sample in at least one analysis in this thesis, and details what each analysis, or line of evidence, suggests about each species hypothesis.

The only New Zealand radicate species clearly monophyletic based on DNA sequence data in this thesis is the subtropical radicate $W$. vernicosa, which incidentally is probably more closely related to other Australian and New Zealand species, and not $W$. littoricola as hypothesised (de Lange and Cameron, 1999) and therefore there is no evidence to support conspecificity between the two. Regarding the other four (temperate) radicate species, the so-called $W$. gracilis complex, there is very little evidence presented in this thesis to suggest that they should not be considered a single species. None of the four species form monophyletic groups in the DNA sequence data. Two do form monophyletic groups in the AFLP tree building analysis (W. akaroa and W. ramosa), but it is difficult to say whether this is representing population level structure or species boundaries, and deserves further investigation with additional sampling.

Within the New Zealand rhizomatous clade two morphologically distinctive rhizomatous species have some level of genetic distinction in both the DNA sequence and AFLP analysis ( $W$. matthewsii and $W$. congesta), which is consistent with their species ranking. A third morphologically-distinctive rhizomatous species, the scree plant $W$. cartilaginea, was only represented by one sample in the AFLP 
analysis, but was found to be monophyletic in the DNA sequencing data and its distinct morphology and habitat all point to recognition at the species level. The other two New Zealand rhizomatous species (W. albomarginata and W. pygmaea) are indistinguishable based on the DNA sequence and AFLP analyses conducted for this thesis. The two species are also indistinguishable based on seed morphology (Webb and Simpson, 2001) and chromosome count (Petterson, 1997b; Petterson et al., 1995). The main character that separates $W$. albomarginata from $W$. pygmaea is the shape of their flowers. W. albomarginata has a 'narrow-campanulate-rotate corolla, with tube distinctly longer than broad' whereas in W. pygmaea the corolla is 'broadly campanulate, with tube as wide as, or wider than long' (Petterson, 1997b). Further study is necessary to determine whether this is simply an artificial North/South Island split between individuals of the same species.

Interestingly although there is no monophyly at the species level several subspecies of both $W$. albomarginata and $W$. pygmaea formed monophyletic groups either in the DNA sequencing analyses or the AFLP analyses (or both). In particular one subspecies of $W$. albomarginata might be a distinct genetic lineage ( $W$. albomarginata subsp. olivina) as the samples included in this thesis consistently formed clades, it can consistently be identified based on morphological characters, and it has a unique habitat (ultramafic rock).

The diversification of the New Zealand rhizomatous group appears to have been driven by local habitat adaptations. Of the distinct lineages, $W$. cartilaginea is found in scree habitats, $W$. matthewsii grows on limestone and $W$. congesta is a coastal plant. The variation between the subspecies of $W$. albomarginata can also be explained by habitat, for example $W$. albomarginata subsp. olivina grows only on ultramafic rock and $W$. albomarginata subsp. flexilis grows on limestone.

Multiple samples of Australian species were not included in the AFLP dataset, so there is less evidence pertaining to species delimitation for them. However, several species were shown to be monophyletic based on DNA sequence data alone, including the rhizomatous $W$. ceracea and the two newly described radicate species (W. rupicola and W. telfordii). Species relationships within the Australian radicate samples are complicated by the fact that they have mostly been shown to contain at least two races of different ploidy levels. Therefore, a dedicated research project into 
the Australian (and New Zealand) radicate species is essential to resolve the taxonomy among these species.

\section{Assessment of molecular techniques used in this thesis}

In this thesis, amplification and sequencing using 22 primer pairs from all three plant genomes was tested on four Wahlenbergia species. Two chloroplast (trnL-F \& trnK) and one nuclear (ITS) marker were selected for phylogenetic reconstruction. These markers were the easiest to amplify, sequence and align and had the highest variability of markers trialed, yet still overall revealed very little genetic variability within the Australasian Wahlenbergia species (Table 3.2). Despite this low genetic variability within Australasian Wahlenbergia the level of variability between Australasian and South African Wahlenbergia using ITS sequences in particular was very high, which made for difficulties in aligning these sequences. This highlights the importance of selecting genetic markers for the particular study in question.

The Australasian phylogeny (Chapter Three) was poorly resolved, due to the low levels of sequence variation, so AFLP analysis was conducted with the hopes that greater variation would be recovered. In the AFLP analysis support values were generally higher towards the tips of the branches than in the DNA sequencing analysis, whereas deeper relationships were equally poorly supported using both AFLP and sequence data, suggesting neither method has the appropriate level of variation to elucidate these relationships. Unfortunately, this means we have not yet managed to discover a molecular technique that is able to resolve deeper relationships within the Australasian Wahlenbergia.

\section{Future directions}

It may be possible to find a more variable DNA marker (such as low copy nuclear genes), which may be able to provide information at the among species level. Although several low-copy nuclear markers trialled in chapter three could not be successfully sequenced, others should be attempted. Alternatively, 'next-generation' sequencing methods could be employed to tackle this question. Next generation 
sequencing methods are high-throughput sequencing technologies that parallelize the sequencing process, producing thousands or millions of sequences at once (Shendure and Ji, 2008). Perhaps more bases will reveal greater numbers of variable sites and hence greater resolution? Another method gaining popularity when traditional methods for finding variable DNA sequence markers has failed is one similar to that taken by Padolina (2006) to design low-copy nuclear markers. Padolina (2006) took a computational approach to design universal angiosperm primers by querying the MoBIoS (Miranker et al., 2003) database to compare the genomes of the monocot $O$. sativa and the eudicot $A$. thaliana to search for primer combinations that occurred only once in each of the two genomes. It is becoming ever more feasible to sequence whole chloroplast genomes and although this has mainly been accomplished for model or crop species such as cotton (Lee et al., 2006) to date, phylogenetic studies using fully sequenced chloroplasts are not unknown (Moore et al., 2007). If the chloroplasts of two closely related Wahlenbergia samples were fully sequenced, it may be possible to design primers for sequences that contained sufficient variation to create resolved phylogenetic trees, and hence resolve deeper relationships within the Australasian Wahlenbergia.

It is also possible that there are no molecular techniques able to resolve the relationships in question, as there may not be the appropriate signal in the genomes. These kinds of molecular techniques rely on mutations that arise by chance, and the probability that mutations will have occurred that are able to correctly assign each individual to a species group must be relatively low, as their radiation (in Australasia) has probably occurred only in the last $3.7 \mathrm{my}$. Thus, some level of fuzziness and uncertainty in delimiting young species at the beginning of differentiation as shown here is therefore perhaps not unexpected (Shaffer and Thompson 2007). A systematic morphological study of the genus, with extensive sampling and accompanying statistical analysis, may even yield more evidence regarding species boundaries than molecular techniques are able to do.

Further research into large-scale relationships of Wahlenbergia should include samples from Asia, the Pacific, South America and Europe. Chromosome counts would be very useful for a larger number of South African species also. At the medium scale (if useful markers can be found) it would be very interesting to assess 
the relationships between the Australian and New Zealand radicate species, particularly the Australian $W$. communis group (which includes the Australian $W$. gracilis), and the New Zealand ' $W$. gracilis' complex (which does not currently contain any species named $W$. gracilis (Webb and Simpson, 2001)). AFLPs have been shown here to be useful at delimiting New Zealand species of Wahlenbergia, and greater sampling of the $W$. gracilis complex, and the $W$. albomarginata/W. pygmaea complex is necessary to tease out relationships within these complexes. There are also outstanding questions relating to two uncollected New Zealand taxa, $W$. congesta subsp. congesta and $W$. albomarginata subsp. decora, which would be good to include in future datasets.

\section{Conclusions}

In this thesis I have reconstructed the first phylogeny of Wahlenbergia, including ca. $20 \%$ of the genus, with samples from its two main areas of diversity, South Africa and Australasia. Wahlenbergia was confirmed to be polyphyletic, although most of these species formed a monophyletic group. Wahlenbergia originated in South Africa, dispersed to and radiated in Australia about 3.7 mya, and from there two introductions to New Zealand are hypothesised, one leading to a rhizomatous clade ca. 1 mya, the other to a radicate group ca. 0.5 mya, thus refuting the hypothesis of Gondwanan vicariance for this group. With regards the New Zealand taxonomy of the group there was no evidence to support the hypothesis that $W$. vernicosa was conspecific with the Australian $W$. littoricola, but also no evidence to refute the hypothesis that all members of the $W$. gracilis complex ( $W$. violacea, $W$. akaroa, $W$. ramosa and $W$. rupestris) were conspecific. There was evidence to support species rank for three morphologically distinct species ( $W$. cartilaginea, $W$. matthewsii and $W$. congesta), but no evidence to refute the conspecificity of the common alpine species $W$. albomarginata and $W$. pygmaea. The nuclear ITS and chloroplast trnL-F and $\operatorname{trnK}$ may not be suitable markers for exploring species relationships within this group, as phylogenies were poorly resolved at deeper levels. However, AFLPs are useful for delimiting New Zealand Wahlenbergia at the species level, and will be more useful for future work. 


\section{References}

Abdel-Monem, A.A., Fernandea, L.A., \& Boone, G.M. 1975. K-Ar ages from the eastern Azores group (Santa Maria, Sao Miguel and the Formigas Islands). Lithos 8:247254.

Albach, D.C. 2007. Amplified fragment length polymorphisms and sequence data in the phylogenetic analysis of polyploids: multiple origins of Veronica cymbalaria (Plantaginaceae). New Phytologist 176:481-498.

Alfaro, M.E., Zoller, S., \& Lutzoni, F. 2003. Bayes or bootstrap? A simulation study comparing the performance of Bayesian Markov chain Monte Carlo sampling and bootstrapping in assessing phylogenetic confidence. Molecular Biology and Evolution 20:255-266.

Allan, H.H. 1961. Flora of New Zealand. Government Printer, Wellington.

Alvarez, I., \& Wendel, J.F. 2003. Ribosomal ITS sequences and plant phylogenetic inference. Molecular Phylogenetics and Evolution 29:417-434.

Antonelli, A. 2008. Higher level phylogeny and evolutionary trends in Campanulaceae subfam. Lobelioideae: Molecular signal overshadows morphology. Molecular Phylogenetics and Evolution 46:1-18.

Bakker, F.T., Breman, F., \& Merckx, V. 2006. DNA sequence evolution in fast evolving mitochondrial DNA nad1 exons in Geraniaceae and Plantaginaceae. Taxon 55:887896.

Bergh, N.G., \& Linder, H.P. 2009. Cape diversification and repeated out-of-southernAfrica dispersal in paper daisies (Asteraceae-Gnaphalieae). Molecular Phylogenetics and Evolution 51:5-18.

Berry, P.E., Hahn, W.J., Sytsma, K.J., Hall, J.C., \& Mast, A. 2004. Phylogenetic relationships and biogeography of Fuchsia (Onagraceae) based on noncoding nuclear and chloroplast DNA data. American Journal of Botany 91:601-614.

Bonin, A., Bellemain, E., Eidesen, P.B., Pompanon, F., Brochmann, C., \& Taberlet, P. 2004. How to track and assess genotyping errors in population genetics studies. Molecular Ecology 13:3261-3273.

Britten, R. 1986. Rates of DNA sequence evolution differ between taxonomic groups. Science 231:1393-1398. 
Broadhurst, L.M., Young, A.G., \& Murray, B.G. 2008. AFLPs reveal an absence of geographical genetic structure among remnant populations of pohutukawa (Metrosideros excelsa, Myrtaceae). New Zealand Journal of Botany 46:13-21.

Bromham, L., \& Penny, D. 2003. The modern molecular clock. Nature Reviews Genetics $4: 216-224$.

Bush, C.M., Wagstaff, S.J., Fritsch, P.W., \& Kron, K.A. 2009. The phylogeny, biogeography and morphological evolution of Gaultheria (Ericaceae) from Australia and New Zealand. Australian Systematic Botany 22:229-242.

Cano-Maqueda, J., Talavera, S., Arista, M., \& Catalan, P. 2008. Speciation and biogeographical history of the Campanula lusitanica complex (Campanulaceae) in the Western Mediterranean region. Taxon 57:11252-11266.

Cellinese, N., Smith, S.A., Edwards, E.J., Kim, S.T., Haberle, R.C., Avramakis, M., \& Donoghue, M.J. 2009. Historical biogeography of the endemic Campanulaceae of Crete. Journal of Biogeography 36:1253-1269.

Chandler, G.T., Plunkett, G.M., Pinney, S.M., Cayzer, L.W., \& Gemmill, C.E.C. 2007. Molecular and morphological agreement in Pittosporaceae: phylogenetic analysis with nuclear ITS and plastid trnL-trnF sequence data. Australian Systematic Botany 20:390-401.

Cho, Y., Mower, J.P., Qiu, Y.L., \& Palmer, J.D. 2004. Mitochondrial substitution rates are extraordinarily elevated and variable in a genus of flowering plants. Proceedings of the National Academy of Sciences of the United States of America 101:1774117746.

Cosner, M.E., Raubeson, L.A., \& Jansen, R.K. 2004. Chloroplast DNA rearrangements in Campanulaceae: phylogenetic utility of highly rearranged genomes. Bmc Evolutionary Biology 4.

Cowan, R.S., Chase, M.W., Kress, W.J., \& Savolainen, V. 2006. 300,000 species to identify: problems, progress, and prospects in DNA barcoding of land plants. Taxon 55:611-616.

Crayn, D.M., Rossetto, M., \& Maynard, D.J. 2006. Molecular phylogeny and dating reveals an Oligo-Miocene radiation of dry-adapted shrubs (former Tremandraceae) from rainforest tree progenitors (Elaeocarpaceae) in Australia. American Journal of Botany 93:1328-1342.

Cuenoud, P., Savolainen, V., Chatrou, L.W., Powell, M., Grayer, R.J., \& Chase, M.W. 2002. Molecular phylogenetics of Caryophyllales based on nuclear 18S rDNA and plastid $r b c L$, atpB, and matK DNA sequences. American Journal of Botany 89:132144. 
Cummings, M.P., Otto, S.P., \& Wakeley, J. 1995. Sampling properties of DNA-sequence data in phylogenetic analysis. Molecular Biology and Evolution 12:814-822.

Cupido, C.N. 2009. Systematic studies of the South African Campanulaceae sensu stricto with an emphasis on generic delimitation. Ph.D. thesis. In. University of Cape Town.

de Lange, P.J. 2005. A comment on Wahlenbergia littoricola subsp. vernicosa. Wellington Botanical Society Newsletter.

de Lange, P.J., \& Cameron, E.K. 1999. The vascular flora of Aorangi Island, Poor Knights Islands, northern New Zealand. New Zealand Journal of Botany 37:433-468.

de Lange, P.J., Norton, D.A., Courtney, S.P., Heenan, P.B., Barkla, J.W., Cameron, E.K., Hitchmough, R., \& Townsend, A.J. 2009. Threatened and uncommon plants of New Zealand (2008 revision). New Zealand Journal of Botany 47:61-96.

De Queiroz, K. 2007. Species concepts and species delimitation. Systematic Biology 56:879-886.

Demesure, B., Sodzi, N., \& Petit, R.J. 1995. A set of universal primers for amplification of polymorphic non-coding regions of mitochondrial and chloroplast DNA in plants Molecular Ecology 4:129-131.

Després, L., Gielly, L., Redoutet, W., \& Taberlet, P. 2003. Using AFLP to resolve phylogenetic relationships in a morphologically diversified plant species complex when nuclear and chloroplast sequences fail to reveal variability. Molecular Phylogenetics and Evolution 27:185-196.

Doyle, J.J., \& Doyle, J.L. 1990. Isolation of plant DNA from fresh tissue. Focus 12:13-15.

Drummond, A.J., Ashton, B., Cheung, M., Heled, J., Kearse, M., Moir, R., Stones-Havas, S., Thierer, T., \& Wilson, A. 2007. Geneious v3.0 available from http://www.geneious.com/.

Drummond, A.J., Ho, S.Y.W., Phillips, M.J., \& Rambaut, A. 2006. Relaxed phylogenetics and dating with confidence. Plos Biology 4:699-710.

Drummond, A.J., \& Rambaut, A. 2007. BEAST: Bayesian evolutionary analysis by sampling trees. Bmc Evolutionary Biology 7.

Eddie, W.M.M., Shulkina, T., Gaskin, J., Haberle, R.C., \& Jansen, R.K. 2003. Phylogeny of Campanulaceae s. str. inferred from its sequences of nuclear ribosomal DNA. Annals of the Missouri Botanical Garden 90:554-575. 
Ennos, R.A., Sinclair, W.T., Hu, X.-S., \& Langdon, A. 1999. Using organelle markers to elucidate the history, ecology and evolution of plant populations. in, Molecular systematics and plant evolution. Taylor and Francis, London.

Erixon, P., Svennblad, B., Britton, T., \& Oxelman, B. 2003. Reliability of Bayesian posterior probabilities and bootstrap frequencies in phylogenetics. Systematic Biology 52:665-673.

Ford, K.A., Ward, J.M., Smissen, R.D., Wagstaff, S.J., \& Breitwieser, I. 2007. Phylogeny and biogeography of Craspedia (Asteraceae : Gnaphalieae) based on ITS, ETS and psbA-trnH sequence data. Taxon 56:783-794.

Forster, G.J.A. 1786. Florulae insularum australium prodromus, Goettingen.

Frajman, B., \& Schneeweiss, G.M. 2009. A Campanulaceous Fate: the Albanian stenoendemic Asyneuma comosiforme in fact belongs to isophyllous Campanula. Systematic Botany 34:595-601.

Gallagher, R.V., Hughes, L., \& Leishman, M.R. 2009. Phenological trends among Australian alpine species: using herbarium records to identify climate-change indicators. Australian Journal of Botany 57:1-9.

Gardner, R.C., De Lange, P.J., Keeling, D.J., Bowala, T., Brown, H.A., \& Wright, S.D. 2004. A late Quaternary phylogeography for Metrosideros (Myrtaceae) in New Zealand inferred from chloroplast DNA haplotypes. Biological Journal of the Linnean Society 83:399-412.

Garnock-Jones, P., Albach, D., \& Briggs, B.G. 2007. Botanical names in Southern Hemisphere Veronica (Plantaginaceae): sect. Detzneria, sect. Hebe, and sect. Labiatoides. Taxon 56:571-582.

Gemmill, C.E.C., Allan, G.J., Wagner, W.L., \& Zimmer, E.A. 2002. Evolution of insular Pacific Pittosporum (Pittosporaceae): Origin of the Hawaiian radiation. Molecular Phylogenetics and Evolution 22:31-42.

Goldblatt, P., Savolainen, V., Porteous, O., Sostaric, I., Powell, M., Reeves, G., Manning, J.C., Barraclough, T.G., \& Chase, M.W. 2002. Radiation in the Cape flora and the phylogeny of peacock irises Moraea (Iridaceae) based on four plastid DNA regions. Molecular Phylogenetics and Evolution 25:341-360.

Gustafsson, M.H.G., \& Bremer, K.r. 1995. Morphology and Phylogenetic Interrelationships of the Asteraceae, Calyceraceae, Campanulaceae, Goodeniaceae, and Related Families (Asterales). American Journal of Botany 82:250-265.

Haase, M., Marshall, B., \& Hogg, I. 2007. Disentangling causes of disjunction on the South Island of New Zealand: the Alpine fault hypothesis of vicariance revisited. Biological Journal of the Linnean Society 91:361-374. 
Haberle, R.C., Dang, A., Lee, T., Penaflor, C., Cortes-Burns, H., Oestreich, A., Raubeson, L., Cellinese, N., Edwards, E.J., Kim, S.T., Eddie, W.M.M., \& Jansen, R.K. 2009. Taxonomic and biogeographic implications of a phylogenetic analysis of the Campanulaceae based on three chloroplast genes. Taxon 58:715-734.

Heenan, P.B., Mitchell, A.D., \& Koch, M. 2002. Molecular systematics of the New Zealand Pachycladon (Brassicaceae) complex: generic circumscription and relationship to Arabidopsis sens. lat. and Arabis sens. lat. New Zealand Journal of Botany 40:543-562.

Hey, J. 2006. On the failure of modern species concepts. Trends in Ecology \& Evolution 21:447-450.

Hillis, D.M., \& Dixon, M.T. 1991. Ribosomal DNA - molecular evolution and phylogenetic inference. Quarterly Review of Biology 66:411-453.

Holland, B., Clarke, A., \& Meudt, H. 2008. Optimizing automated AFLP scoring parameters to improve phylogenetic resolution. Systematic Biology 57:347-366.

Houliston, G.J., \& Olson, M.S. 2006. Nonneutral evolution of organelle genes in Silene vulgaris. Genetics 174:1983-1994.

Hudson, R.R., \& Coyne, J.A. 2002. Mathematical consequences of the genealogical species concept. Evolution 56:1557-1565.

Huelsenbeck, J.P., Ronquist, F. . 2001. MRBAYES: Bayesian inference of phylogeny. Bioinformatics 17: 754-755.

Hughes, C.E., Eastwood, R.J., \& Bailey, C.D. 2006. From famine to feast? Selecting nuclear DNA sequence loci for plant species-level phylogeny reconstruction. Philosophical Transactions of the Royal Society B-Biological Sciences 361:211225.

Hurr, K.A., Lockhart, P.J., Heenan, P.B., \& Penny, D. 1999. Evidence for the recent dispersal of Sophora (Leguminosae) around the Southern Oceans: molecular data. Journal of Biogeography 26:565-577.

Huson, D.H., \& Bryant, D. 2006. Application of phylogenetic networks in evolutionary studies. Molecular Biology and Evolution 23:254-267.

Ishida, T.A., Hattori, K., Sato, H., \& Kimura, M.T. 2003. Differentiation and hybridization between Quercus crispula and Q. dentata (Fagaceae): Insights from morphological traits, amplified fragment length polymorphism markers, and leafminer composition. American Journal of Botany 90:769-776. 
Joly, S., Heenan, P.B., \& Lockhart, P.J. 2009. A Pleistocene inter-tribal allopolyploidization event precedes the species radiation of Pachycladon (Brassicaceae) in New Zealand. Molecular Phylogenetics and Evolution 51:365372.

Kamp, P.J.J. 1986. Late Cretaceous-Cenozoic tectonic development of the southwest pacific region. Tectonophysics 121:225-251.

Kelchner, S.A. 2000. The evolution of non-coding chloroplast DNA and its application in plant systematics. Annals of the Missouri Botanical Garden.

Knapp, M., Mudaliar, R., Havell, D., Wagstaff, S.J., \& Lockhart, P.J. 2007. The drowning of New Zealand and the problem of Agathis. Systematic Biology 56:862-870.

Kochanek, J., Steadman, K.J., Probert, R.J., \& Adkins, S.W. 2009. Variation in seed longevity among different populations, species and genera found in collections from wild Australian plants. Australian Journal of Botany 57:123-131.

Koopman, M.M., \& Ayers, T.J. 2005. Nectar spur evolution in the Mexican lobelias (Campanulaceae : Lobelioideae). American Journal of Botany 92:558-562.

Koopman, W.J.M. 2005. Phylogenetic signal in AFLP data sets. Systematic Biology 54:197-217.

Koopman, W.J.M., Wissemann, V., De Cock, K., Van Huylenbroeck, J., De Riek, J., Sabatlno, G.J.H., Visser, D., Vosman, B., Ritz, C.M., Maes, B., Werlemark, G., Nybom, H., Debener, T., Linde, M., \& Smulders, M.J.M. 2008. AFLP markers as a tool to reconstruct complex relationships: A case study in Rosa (Rosaceae). American Journal of Botany 95:353-366.

Kosman, E., \& Leonard, K.J. 2005. Similarity coefficients for molecular markers in studies of genetic relationships between individuals for haploid, diploid, and polyploid species. Molecular Ecology 14:415-424.

Lammers, T.G. 1996. Phylogeny, biogeography, and systematics of the Wahlenbergia fernandeziana complex (Campanulaceae: Campanuloideae). Systematic Botany 21:397-415.

Lee, C., \& Wen, J. 2004. Phylogeny of Panax using chloroplast trnC-trnD intergenic region and the utility of $t r n C-t r n D$ in interspecific studies of plants. Molecular Phylogenetics and Evolution 31:894-903.

Lee, S.-B., Kaittanis, C., Jansen, R., Hostetler, J., Tallon, L., Town, C., \& Daniell, H. 2006. The complete chloroplast genome sequence of Gossypium hirsutum: organization and phylogenetic relationships to other angiosperms. BMC Genomics $7: 61$. 
Levin, R.A., Blanton, J., \& Miller, J.S. 2009. Phylogenetic utility of nuclear nitrate reductase: A multi-locus comparison of nuclear and chloroplast sequence data for inference of relationships among American Lycieae (Solanaceae). Molecular Phylogenetics and Evolution 50:608-617.

Lewis, K.B., Carter, L., \& Davey, F.J. 1994. The opening of cook strait - interglacial tidal scour and aligning basins at a subduction to transform plate edge Marine Geology 116:293-312.

Li, M., Wunder, J., Bissoli, G., Scarponi, E., Gazzani, S., Barbaro, E., Saedler, H., \& Varotto, C. 2008. Development of COS genes as universally amplifiable markers for phylogenetic reconstructions of closely related plant species. Cladistics 24:727745 .

Lloyd, D.G., \& Yates, J.M.A. 1982. Intra-sexual selection and the segregation of pollen and stigmas in hermaphrodite plants, exemplified by Wahlenbergia albomarginata (Campanulaceae). Evolution 36:903-913.

Lockhart, P.J., McLenachan, P.A., Harell, D., Glenny, D., Huson, D., \& Jensen, U. 2001. Phylogeny, radiation, and transoceanic dispersal of New Zealand alpine buttercups: Molecular evidence under split decomposition. Annals of the Missouri Botanical Garden 88:458-477.

Lundberg, J., \& Bremer, K. 2003. A phylogenetic study of the order Asterales using one morphological and three molecular data sets. International Journal of Plant Sciences 164:553-578.

Luo, R.Y., Hipp, A.L., \& Larget, B. 2007. A bayesian model of AFLP marker evolution and phylogenetic inference. Statistical Applications in Genetics and Molecular Biology 6.

MacPhail, M.K. 1997. The New Zealand flora - Entirely long-distance dispersal? Comment. Journal of Biogeography 24:113-117.

Maddison, D.R., Maddison, W.P. 2001. MacClade 4. In. Sinauer Associates, Sunderland.

Mayden, R.L. 1997. A hierarchy of species concepts: The denouement in the saga of the species problem. Pp. 381-424 in: Claridge, M.F., Dawah, H.A., \& Wilson, M.R., (eds), Species: The units of biodiversity. Chapman and Hall, London. p 381-424.

Mayr, E. 1942. Systematics and origin of species. Columbia University Press, New York.

Meudt, H., \& Bayly, M. 2008. Phylogeographic patterns in the Australasian genus Chionohebe (Veronica sl, Plantaginaceae) based on AFLP and chloroplast DNA sequences. Molecular Phylogenetics and Evolution 47:319-338. 
Meudt, H., \& Clarke, A. 2007. Almost forgotten or latest practice? AFLP applications, analyses and advances. Trends in Plant Science 12:106-117.

Meudt, H., Lockhart, P., \& Bryant, D. 2009. Species delimitation and phylogeny of a New Zealand plant species radiation. BMC Evol Biol 9:111.

Meudt, H.M., \& Simpson, B.B. 2006. The biogeography of the austral, subalpine genus Ourisia (Plantaginaceae) based on molecular phylogenetic evidence: South American origin and dispersal to New Zealand and Tasmania. Biological Journal of the Linnean Society 87:479-513.

Miranker, D., Xu, W., \& Mao, R. 2003. MoBIoS: A Metric-Space DBMS to Support Biological Discovery. In: 15th International Conference on Scientific and Statistical Database Management. p 241.

Mitchell, A.D., \& Heenan, P.B. 2002. Sophora sect. Edwardsia (Fabaceae): further evidence from nrDNA sequence data of a recent and rapid radiation around the Southern Oceans. Botanical Journal of the Linnean Society 140:435-441.

Moore, M.J., Bell, C.D., Soltis, P.S., \& Soltis, D.E. 2007. Using plastid genome-scale data to resolve enigmatic relationships among basal angiosperms. Proceedings of the National Academy of Sciences of the United States of Amercia 104 19363-19368.

Morgan-Richards, M., Smissen, R.D., Shepherd, L.D., Wallis, G.P., Hayward, J.J., Chan, C.H., Chambers, G.K., \& Chapman, H.M. 2009. A review of genetic analyses of hybridisation in New Zealand. Journal of the Royal Society of New Zealand 39:1534.

Mueller, U.G., \& Wolfenbarger, L.L. 1999. AFLP genotyping and fingerprinting. Trends in Ecology \& Evolution 14:389-394.

Muller, J. 1981. Fossil pollen records of extant angiosperms. Botanical Revue 47.

Murray, B.G., Datson, P.M., Lai, E.L., Sheath, K.M., \& Cameron, E.K. 2004. Polyploidy, hybridization and evolution in Pratia (Campanulaceae). New Zealand Journal of Botany 42:905-920.

Nei, M., \& Kumar, S. 2000. Molecular evolution and phylogenetics. Oxford University Press, New York.

Nicolson, D.H., \& Fosberg, D.R. 2004. The Forsters and the botany of the second Cook expedition (1772-1775) 2ed. Gantner Verlag, Vienna.

Odhav, B., Beekrum, S., Akula, U., \& Baijnath, H. 2007. Preliminary assessment of nutritional value of traditional leafy vegetables in KwaZulu-Natal, South Africa. Journal of Food Composition and Analysis 20:430-435. 
Oxelman, B., Liden, M., \& Berglund, D. 1997. Chloroplast rps16 intron phylogeny of the tribe Sileneae (Caryophyllaceae). Plant Systematics and Evolution 206:393-410.

Padolina, J.M. 2006. Phylogenetic Reconstruction of Phalaenopsis Using Nuclear and Chloroplast DNA Sequence Data and Using Phalaenopsis as a Natural System for Assessing Methods to Reconstruct Hybrid Evolution in Phylogenetic Analyses. $\mathrm{PhD}$ Thesis. In. The University of Texas at Austin.

Park, J.M., Kovacic, S., Liber, Z., Eddie, W.M.M., \& Schneeweiss, G.M. 2006. Phylogeny and biogeography of isophyllous species of Campanula (Campanulaceae) in the Mediterranean area. Systematic Botany 31:862-880.

Peralta, I., \& Spooner, D. 2001. Granule-bound starch synthase (GBSSI) gene phylogeny of wild tomatoes (Solanum L. section Lycopersicon [Mill.] Wettst. subsection Lycopersicon) 1. American Journal of Botany 88:1888-1902.

Perez-Espona, S., Sales, F., Hedge, I., \& Moller, M. 2005. Phylogeny and species relationships in Jasione (Campanulaceae) with emphasis on the 'montana' complex. Edinburgh Journal of Botany 62:29-51.

Perrie, L.R., Brownsey, P.J., Lockhart, P.J., Brown, E.A., \& Large, M.F. 2003. Biogeography of temperate Australasian Polystichum ferns as inferred from chloroplast sequence and AFLP. Journal of Biogeography 30:1729-1736.

Perrie, L.R., \& Shepherd, L.D. 2009. Reconstructing the species phylogeny of Pseudopanax (Araliaceae), a genus of hybridising trees. Molecular Phylogenetics and Evolution 52:774-783.

Peter, C.I., \& Johnson, S.D. 2008. Mimics and magnets: The importance of color and ecological facilitation in floral deception. Ecology 89:1583-1595.

Petit, C., Bretagnolle, F., \& Felber, F. 1999. Evolutionary consequences of diploidpolyploid hybrid zones in wild species. Trends in Ecology \& Evolution 14:306-311.

Petterson, J. 1953. The genus Wahlenbergia in New Zealand. Unpublished MSc thesis In. Victoria University of Wellington.

Petterson, J. 2005. The genus Wahlenbergia, (Campanulaceae): the harebells of New Zealand. Wellington Botanical Society Bulletin 49:16 - 35.

Petterson, J.A. 1997a. Identity of the original Wahlenbergia gracilis (Campanulaceae) and allied species. An historical review of early collections. New Zealand Journal of Botany 35:55-78.

Petterson, J.A. 1997b. Revision of the genus Wahlenbergia (Campanulaceae) in New Zealand. New Zealand Journal of Botany 35:9-54. 
Petterson, J.A., Williams, E.G., \& Dawson, M.I. 1995. Contributions to a chromosome atlas of the New Zealand flora .34. Wahlenbergia (Campanulaceae). New Zealand Journal of Botany 33:489-496.

Plunkett, G.T., Bruhl, J.J., \& Telford, I.R.H. 2009. Two new, sympatric species of (Campanulaceae) from the New England Tableland escarpment, New South Wales, Australia. Australian Systematic Botany 22:319-331.

Pole, M. 1994. The New Zealand Flora - entirely long-distance dispersal. Journal of Biogeography 21:625-635.

Posada, D. 2008. jModelTest: Phylogenetic Model Averaging. Molecular Biology and Evolution 25:1253-1256.

Prebble, M., Sim, R., Finn, J., \& Fink, D. 2005. A Holocene pollen and diatom record from Vanderlin Island, Gulf of Carpentaria, lowland tropical Australia. Quaternary Res 64:357-371.

Rambaut, A., \& Drummond, A.J. 2007. Tracer v1.4 available at: http://beast.bio.ed.ac.uk/Tracer. In.

Raven, P.H. 1973. Evolution of subalpine and alpine plant groups in New Zealand New Zealand Journal of Botany 11:177-200.

Reeves, G., Chase, M., Goldblatt, P., Rudall, P., Fay, M., Cox, A., Lejeune, B., \& SouzaChies, T. 2001. Molecular systematics of Iridaceae: evidence from four plastid DNA regions 1. American Journal of Botany 88:2074-2087.

Rivadavia, F., Kondo, K., Kato, M., \& Hasebe, M. 2003. Phylogeny of the sundews, Drosera (Droseraceae), based on chloroplast $r b c L$ and nuclear 18S ribosomal DNA sequences. American Journal of Botany 90:123-130.

Rohlf, F.J. 1990. NTSYS-pc: Numerical Taxonomy and Multivariate Analysis System. Applied Biostatistics, Setauket, New York.

Roquet, C., Saez, L., Aldasoro, J.J., Susanna, A., Alarcon, M.L., \& Garcia-Jacas, N. 2008. Ural Delineation, Molecular Phylogeny and Floral Evolution in Campanula. Systematic Botany 33:203-217.

Roquet, C., Sanmartin, I., Garcia-Jacas, N., Saez, L., Susanna, A., Wikstrom, N., \& Aldasoro, J.J. 2009. Reconstructing the history of Campanulaceae with a Bayesian approach to molecular dating and dispersal-vicariance analyses. Molecular Phylogenetics and Evolution 52:575-587.

Roth, A.W. 1821. Novae plantarum species praeserim Indiae orientalis. 
Sanderson, M.J., Thorne, J.L., Wikstrom, N., \& Bremer, K. 2004. Molecular evidence on plant divergence times. American Journal of Botany 91:1656-1665.

Sang, T., Crawford, D.J., \& Stuessy, T.F. 1997. Chloroplast DNA phylogeny, reticulate evolution, and biogeography of Paeonia (Paeoniaceae). In: American Journal of Botany. p 1120-1136.

Sanmartín, I., Wanntorp, L., \& Winkworth, R.C. 2007. West Wind Drift revisited: testing for directional dispersal in the Southern Hemisphere using event-based tree fitting. Journal of Biogeography 34:398-416.

Schrader, H.A. 1814. Plantarum semina in Horto Academico Gottingensi, Anno 1814 collecta.

Shaffer, H., \& Thomson, R. 2007. Delimiting Species in Recent Radiations. Systematic Biology 56:896-906.

Shaw, J., Lickey, E.B., Beck, J.T., Farmer, S.B., Liu, W.S., Miller, J., Siripun, K.C., Winder, C.T., Schilling, E.E., \& Small, R.L. 2005. The tortoise and the hare II: Relative utility of 21 noncoding chloroplast DNA sequences for phylogenetic analysis. In: American Journal of Botany. p 142-166.

Shendure, J., \& Ji, H. 2008. Next-generation DNA sequencing. Nature Biotechnology 26:1135-1145.

Shepherd, L.D., Perrie, L.R., \& Brownsey, P.J. 2008. Low-copy nuclear DNA sequences reveal a predominance of allopolyploids in a New Zealand Asplenium fern complex. Molecular Phylogenetics and Evolution 49:240-248.

Simpson, G. 1945. Notes on some New Zealand plants and descriptions of new species (No. 4). Transactions of the Royal Society of New Zealand 75:187-202.

Simpson, G. 1952. Notes on some New Zealand plants and descriptions of new species (No. 5). Transactions and Proceedings of the Royal Society of New Zealand 79:419-435.

Small, R.L., Cronn, R.C., \& Wendel, J.F. 2004. Use of nuclear genes for phylogeny reconstruction in plants. Australian Systematic Botany 17:145-170.

Smissen, R., \& Breitwieser, I. 2008. Species relationships and genetic variation in the New Zealand endemic Leucogenes (Asteraceae: Gnaphalieae). New Zealand Journal of Botany 46:65-76.

Smissen, R.D., Breitwieser, I., \& Ward, J.M. 2004. Phylogenetic implications of transspecific chloroplast DNA sequence polymorphism in New Zealand Gnaphalieae (Asteraceae). Plant Systematics and Evolution 249:37-53. 
Smith, A.B., \& Peterson, K.J. 2002. Dating the time of origin of major clades: molecular clocks and the fossil record. Annual Review of Earth and Planetary Sciences 30:6588 .

Smith, P.J. 1992. A revision of the genus Wahlenbergia (Campanulaceae) in Australia. Telopea 5:91-175.

Soltis, E.D., \& Soltis, P.S. 2000. Contributions of plant molecular systematics to studies of molecular evolution. Plant Molecular Biology 42:45-75.

Soltis, P.S., \& Soltis, D.E. 2009. The role of hybridization in plant speciation. Annual Review of Plant Biology 60:561-588.

Steele, P.R., Guisinger-Bellian, M., Linder, C.R., \& Jansen, R.K. 2008. Phylogenetic utility of 141 low-copy nuclear regions in taxa at different taxonomic levels in two distantly related families of rosids. Molecular Phylogenetics and Evolution 48:1013-1026.

Sterck, L., Rombauts, S., Vandepoele, K., Rouze, P., \& Van de Peer, Y. 2007. How many genes are there in plants (. . . and why are they there)? Current Opinion in Plant Biology 10:199-203.

Stöckler, K., Daniel, I.L., \& Lockhart, P.J. 2002. New Zealand Kauri (Agathis australis (D. don) Lindl., Araucariaceae) survives oligocene drowning. Systematic Biology 51:827-832.

Swofford, D.L. 2002. PAUP*. Phylogenetic Analysis Using Parsimony (*and Other Methods). Version 4. In. Sinauer Associates Sunderland, Massachusetts.

Taberlet, P., Gielly, L., Pautou, G., \& Bouvet, J. 1991. Universal primers for amplification of three non-coding regions of chloroplast DNA. Plant Molecular Biology.

Tate, J.A., \& Simpson, B.B. 2003. Paraphyly of Tarasa (Malvaceae) and diverse origins of the polyploid species. In: Syst Bot. p 723-737.

Tay, M.L., Meudt, H.M., Garnock-Jones, P.J., \& Ritchie, P.A. 2010. DNA sequences from three genomes reveal multiple long-distance dispersals and non-monophyly of sections in Australasian Plantago (Plantaginaceae). Australian Systematic Botany 23:47-68.

Thorsen, M.J., Dickinson, K.J.M., \& Seddon, P.J. 2009. Seed dispersal systems in the New Zealand flora. Perspectives in Plant Ecology, Evolution and Systematics 11:285309. 
Trewick, S.A., \& Morgan-Richards, M. 2005. After the deluge: mitochondrial DNA indicates Miocene radiation and Pliocene adaptation of tree and giant weta (Orthoptera : Anostostomatidae). Journal of Biogeography 32:295-309.

Trewick, S.A., Paterson, A.M., \& Campbell, H.J. 2007. Guest Editorial: Hello New Zealand. Journal of Biogeography.

van Steenis, C.G.G.J. 1960. Original generic description and typification of the genus 8668 Wahlenbergia Schrad. ex Roth (1921) Campanulaceae. Taxon 9:122.

Vos, P., Hogers, R., Bleeker, M., Reijans, M., Vandelee, T., Hornes, M., Frijters, A., Pot, J., Peleman, J., Kuiper, M., \& Zabeau, M. 1995. AFLP - a new technique for DNAfingerprinting Nucleic Acids Research 23:4407-4414.

Vriesendorp, B., \& Bakker, F.T. 2005. Reconstructing patterns of reticulate evolution in angiosperms: what can we do? Taxon 54:593-604.

Wagstaff, S.J., Bayly, M.J., \& Garnock-Jones, P.J. 2002. Classification, origin, and diversification of the New Zealand hebes (Scrophulariaceae). Annals of the Missouri Botanical Garden.

Wagstaff, S.J., \& Garnock-Jones, P.J. 1998. Evolution and biogeography of the Hebe complex (Scrophulariaceae) inferred from ITS sequences. New Zealand Journal of Botany 36:425-437.

Wagstaff, S.J., \& Garnock-Jones, P.J. 2000. Patterns of diversification in Chionohebe and Parahebe (Scrophulariaceae) inferred from ITS sequences. New Zealand Journal of Botany 38:389-407.

Wagstaff, S.J., Heenan, P.B., \& Sanderson, M.J. 1999. Classification, origins, and patterns of diversification in New Zealand Carmichaelinae (Fabaceae). American Journal of Botany 86:1346-1356.

Wagstaff, S.J., Molloy, B.P.J., \& Tate, J.A. 2010. Evolutionary significance of long distance dispersal and hybridisation in the New Zealand endemic genus Hoheria (Malvaceae). in Press.

Wallis, G.P., \& Trewick, S.A. 2009. New Zealand phylogeography: evolution on a small continent. Molecular Ecology 18:3548-3580.

Walsh, N.G. 1999. Wahlenbergia. in: Walsh, N.G., Entwisle, T.J, (ed), Flora of Victoria Volume 4: Dicotyledons (Cornanceae to Asteraceae). Inkata Press, Australia.

Wardle, P. 1963. Evolution and distribution of the New Zealand flora, as affected by quaternary climates. New Zealand Journal of Botany 1:3-17. 
Waters, J.M., \& Roy, M.S. 2004. Out of Africa: The slow train to Australasia. Systematic Biology 53:18-24.

Webb, C.J., \& Simpson, M.J.A. 2001. Seeds of New Zealand gymnosperms \& dicotyledons. Manuka Press, Christchurch.

White, T. 1990. Amplification and direct sequencing of fungal ribosomal RNA genes for phylogenetics. Pp. 315-322 in: M Innis, C.G., J Sninsky, T White, (ed), PCR protocols: a guide to methods and applications. Academic Press, San Diego. p 315322.

Wikström, N., Savolainen, V., \& Chase, M.W. 2001. Evolution of the angiosperms: calibrating the family tree. Proceedings of the Royal Society B-Biological Sciences 268:2211-2220.

Wikström, N., Savolainen, V., \& Chase, M.W. 2003. Angiosperm divergence times: congruence and incongruence between fossils and sequence divergence estimates. Pp. 142-165 in: Donoghue, P.C.J., \& Smith, M.P., (eds), Telling the Evolutionary Time: Molecular Clocks and the Fossil Record. p 142-165.

Wilcox, T.P., Zwickl, D.J., Heath, T.A., \& Hillis, D.M. 2002. Phylogenetic relationships of the dwarf boas and a comparison of Bayesian and bootstrap measures of phylogenetic support. Molecular Phylogenetics and Evolution 25:361-371.

Winkworth, Robertson, A.W., \& Ehrendorfer, F. 1999. The importance of dispersal and recent speciation in the flora of New Zealand. Journal of Biogeography.

Winkworth, R., Grau, J., Robertson, A., \& Lockhart, P. 2002a. The origins and evolution of the genus Myosotis L.(Boraginaceae). Molecular Phylogenetics and Evolution 24:180-193.

Winkworth, R., Wagstaff, S.J., Glenny, D., \& Lockhart, P. 2002b. Plant dispersal NEWS from New Zealand. Trends in Ecology \& Evolution 17:514-520.

Winkworth, R.C., Wagstaff, S.J., \& Glenny, D. 2005. Evolution of the New Zealand mountain flora: Origins, diversification and dispersal. Organisms Diversity \& Evolution.

Wolfe, A.D., \& Randle, C.P. 2004. Recombination, heteroplasmy, haplotype polymorphism, and paralogy in plastid genes: implications for plant molecular systematics. Systematic Botany 29:1011-1020.

Woo, V., L. . 2007. Evolution, biogeography and taxonomy of the southwest Pacific Tribe Coronanthereae (Gesneriaceae). PhD thesis In: Botany. Victoria University of Wellington. 
Woolley, S.M., Posada, D., \& Crandall, K.A. 2008. A Comparison of Phylogenetic Network Methods Using Computer Simulation. Plos One 3.

Yoder, A.D., Irwin, J.A., \& Payseur, B.A. 2001. Failure of the ILD to determine data combinability for slow loris phylogeny. Systematic Biology 50:408-424.

Zimmer, E., Roalson, E., Skog, L., Boggan, J., \& Idnurm, A. 2002. Phylogenetic relationships in the Gesnerioideae (Gesneriaceae) based on nrDNA ITS and cpDNA trn LF and trn ET spacer region sequences 1. American Journal of Botany 89:296-311.

Zwickl, D.J. 2006. Genetic algorithm approaches for the phylogenetic analysis of large biological sequence data sets under the maximum likelihood criterion. Ph.D. Thesis In. The University of Texas Austin. 


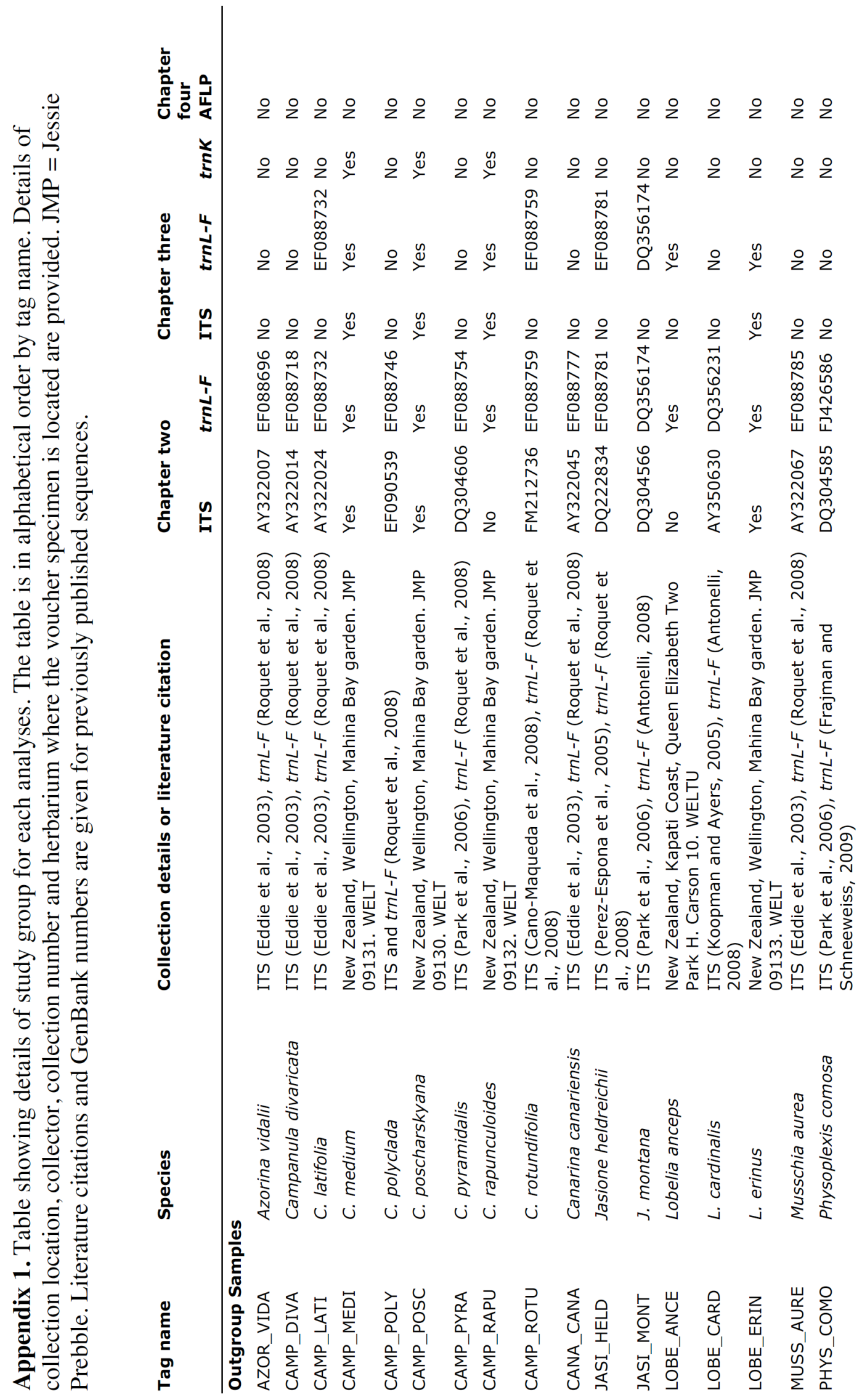




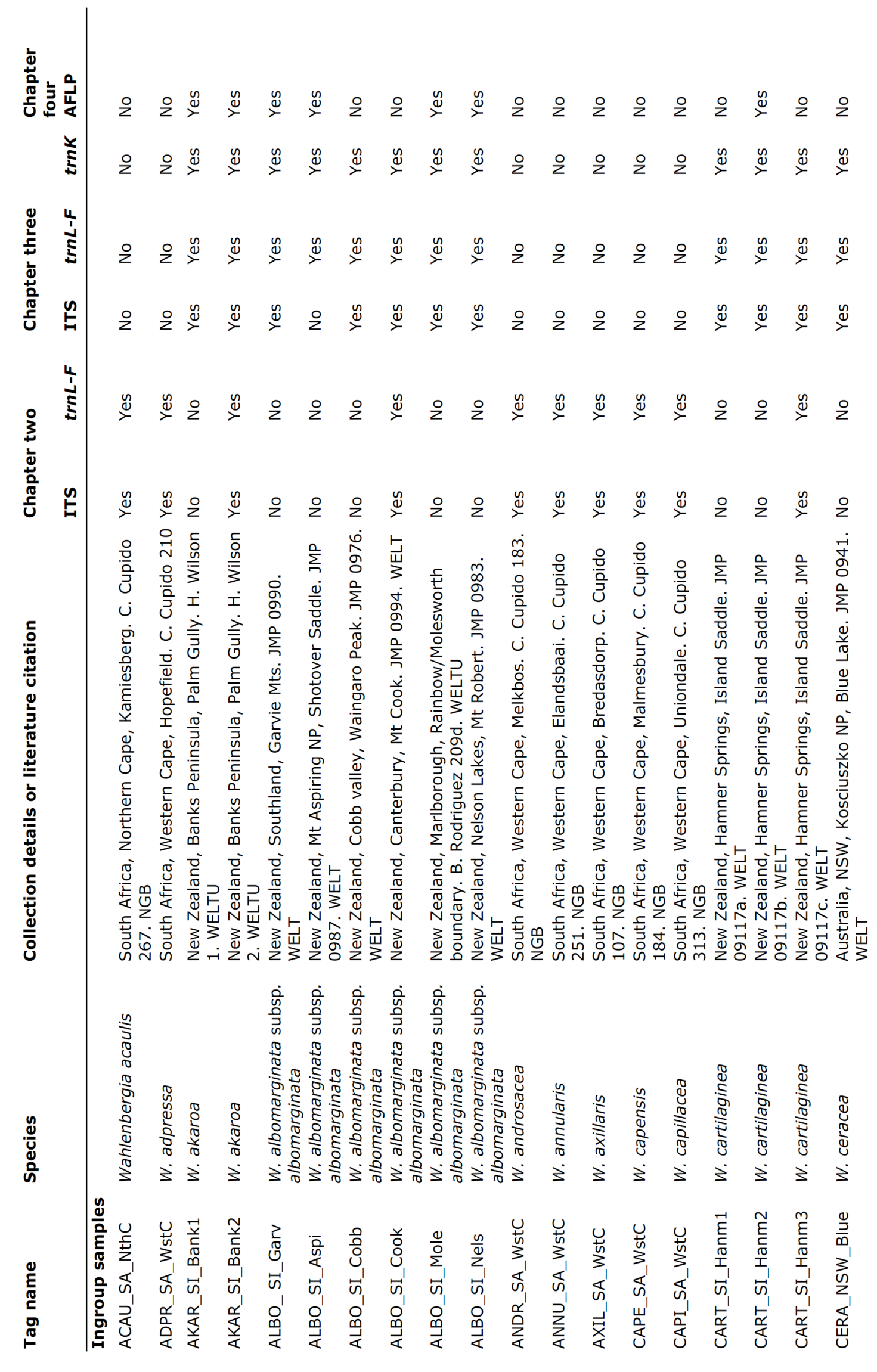




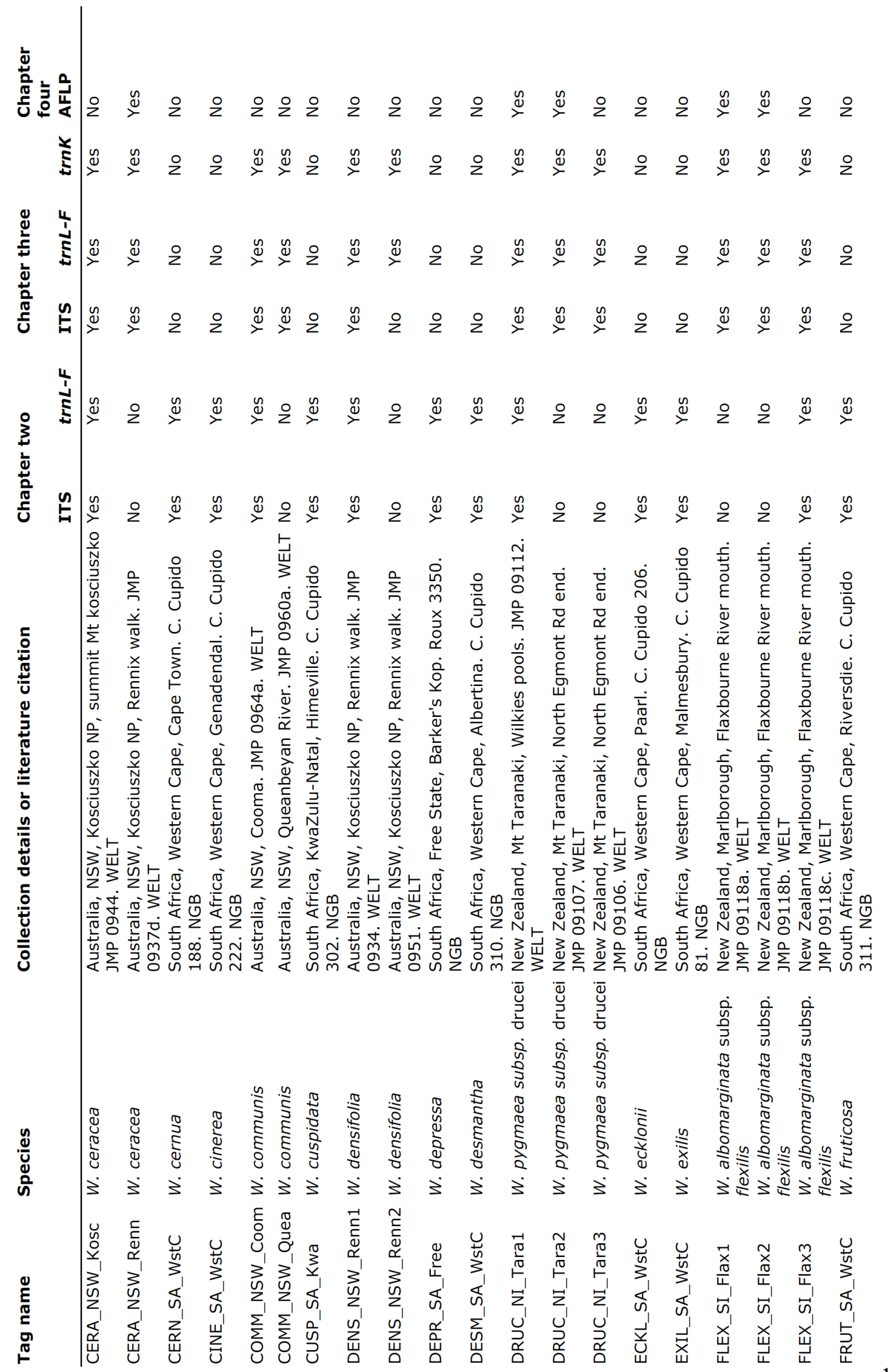




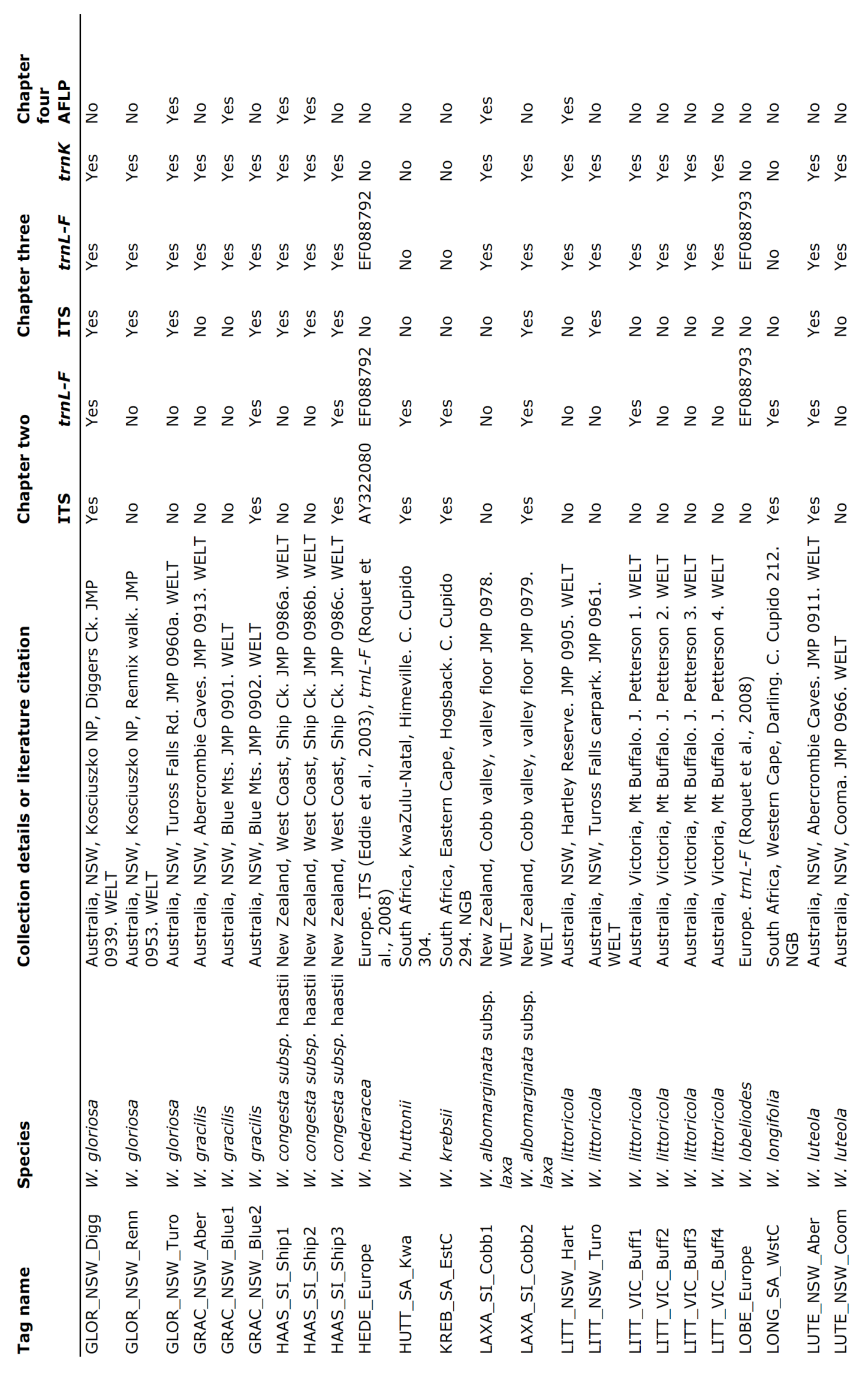




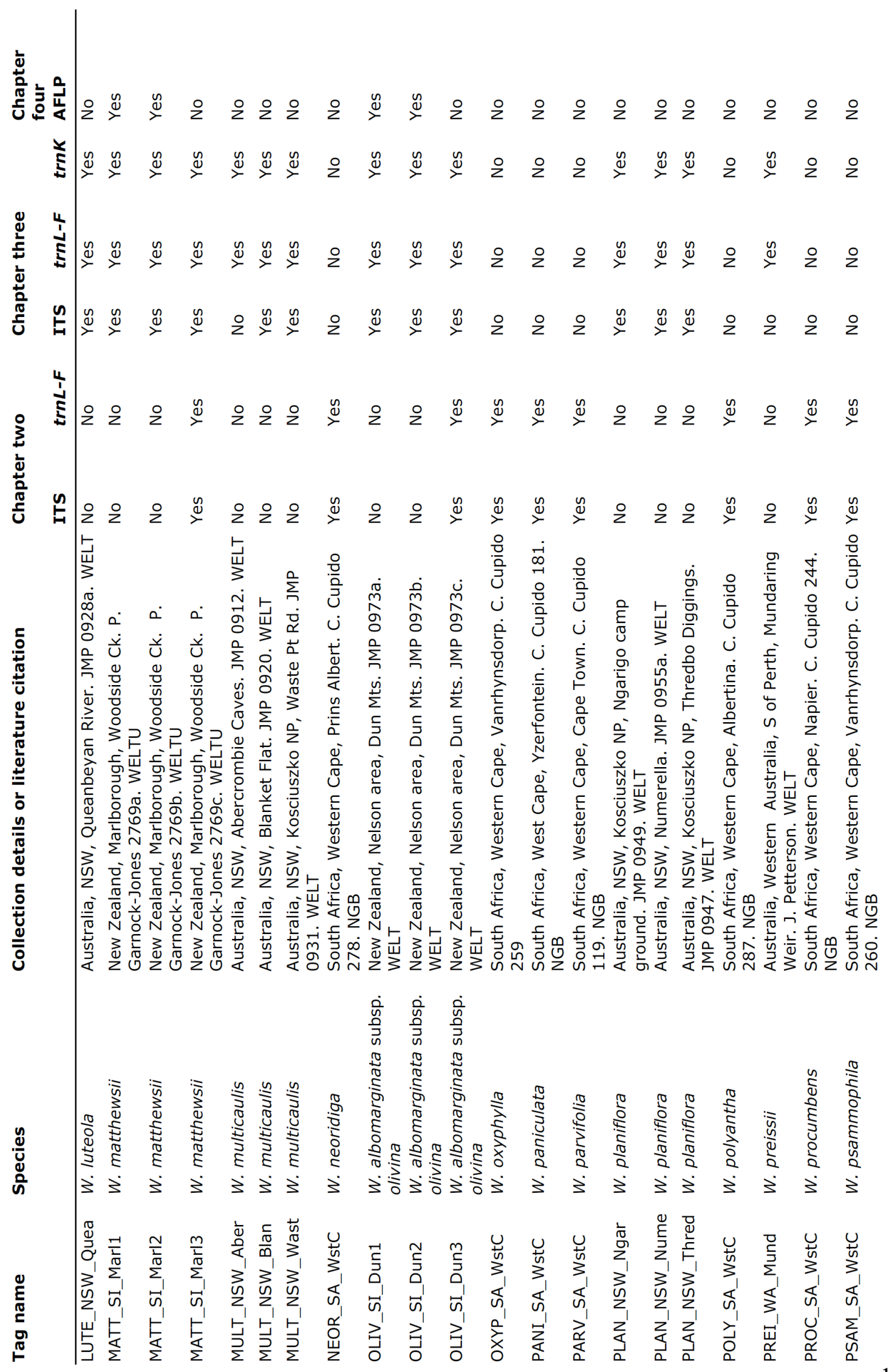




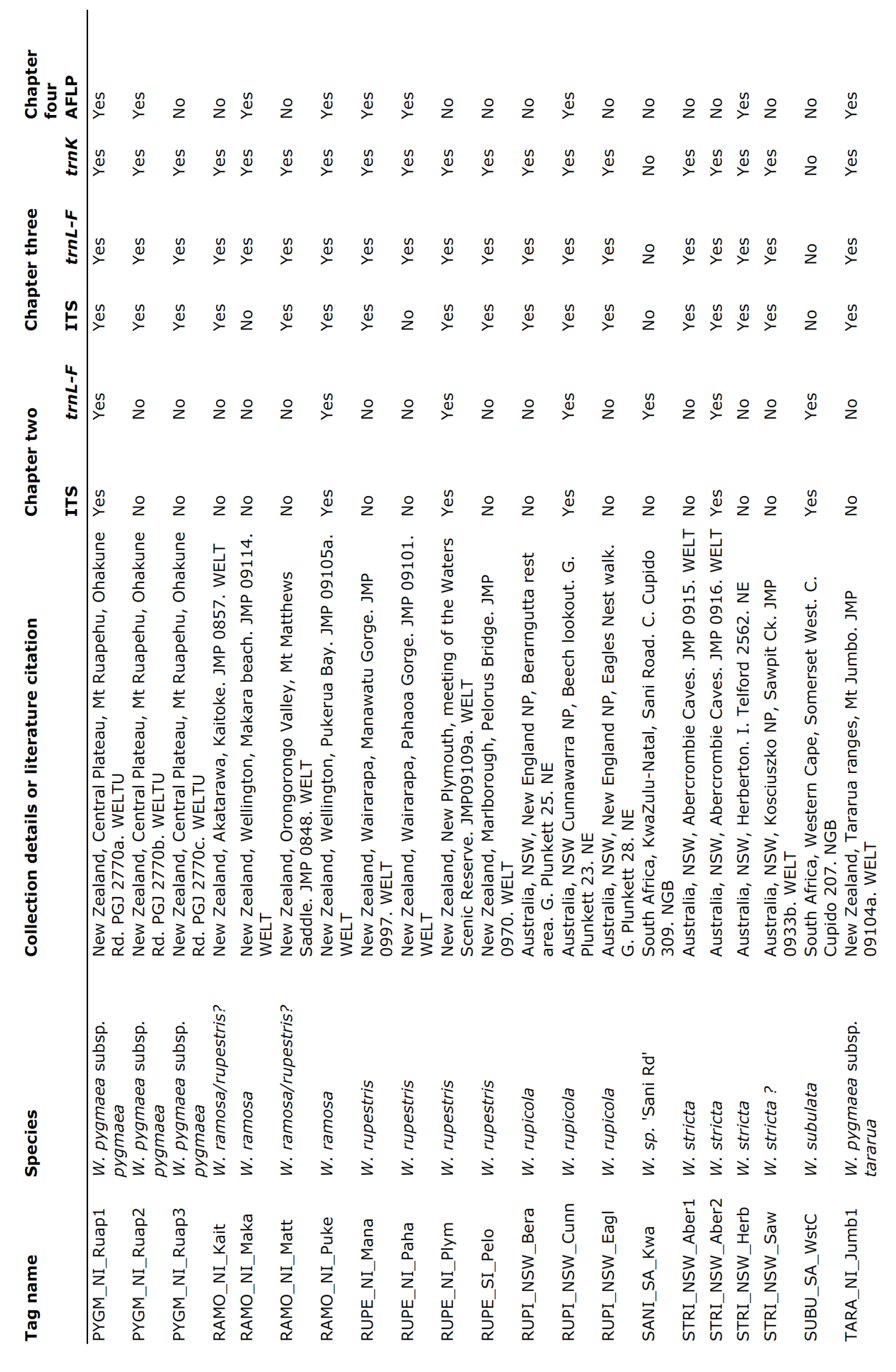




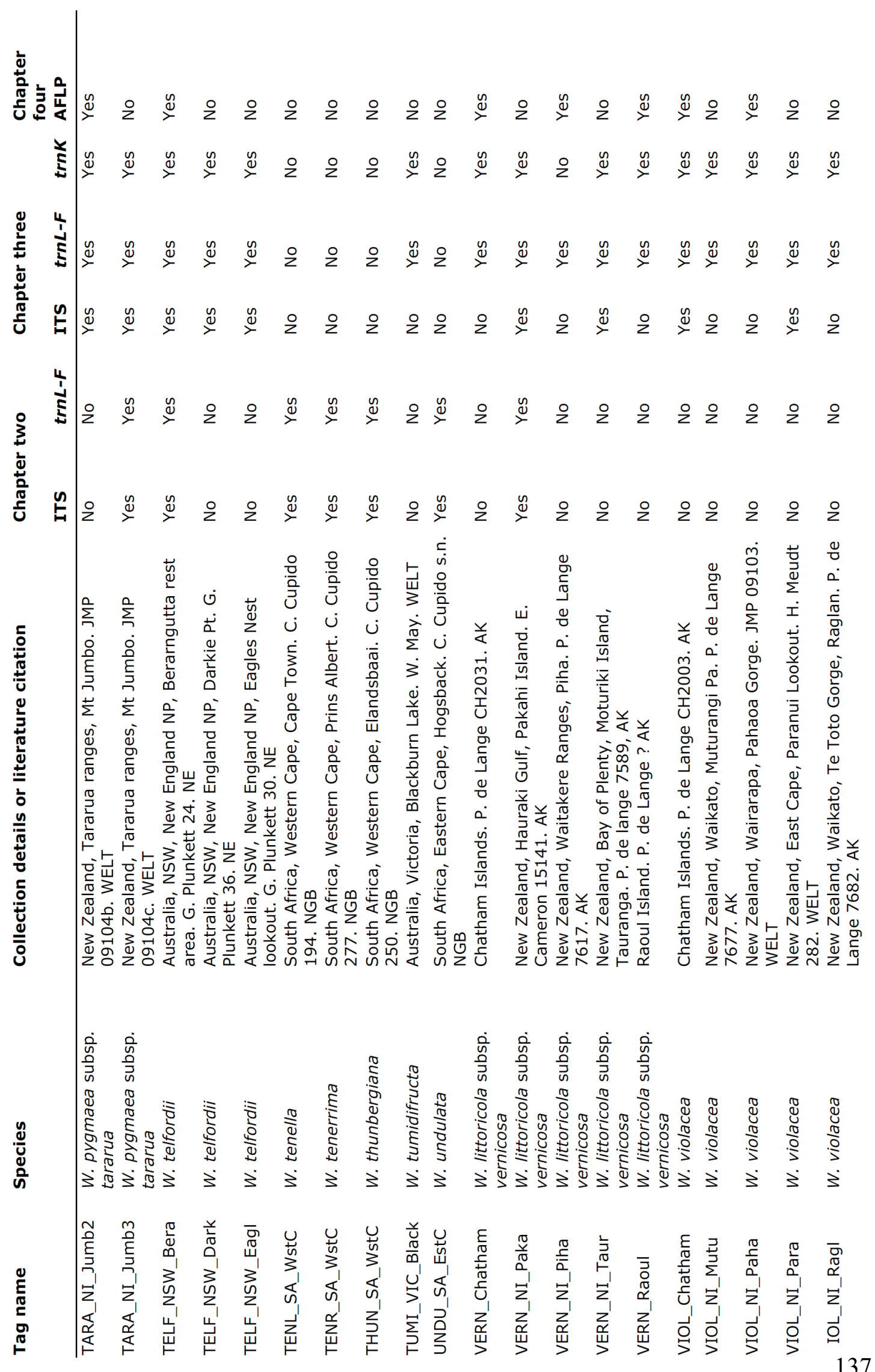




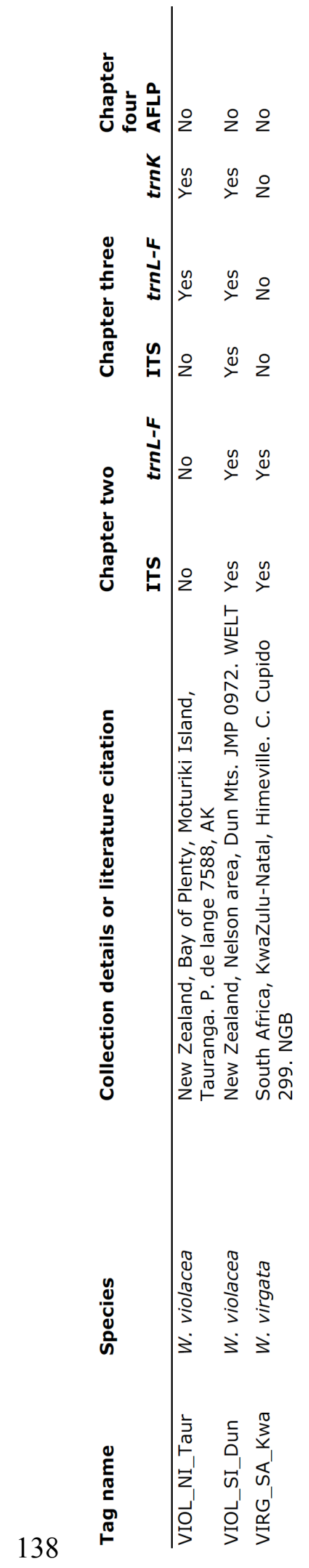


Appendix 2.1 Neighbour net analysis of the ITS dataset from Chapter Three created using SplitsTree v 4.10

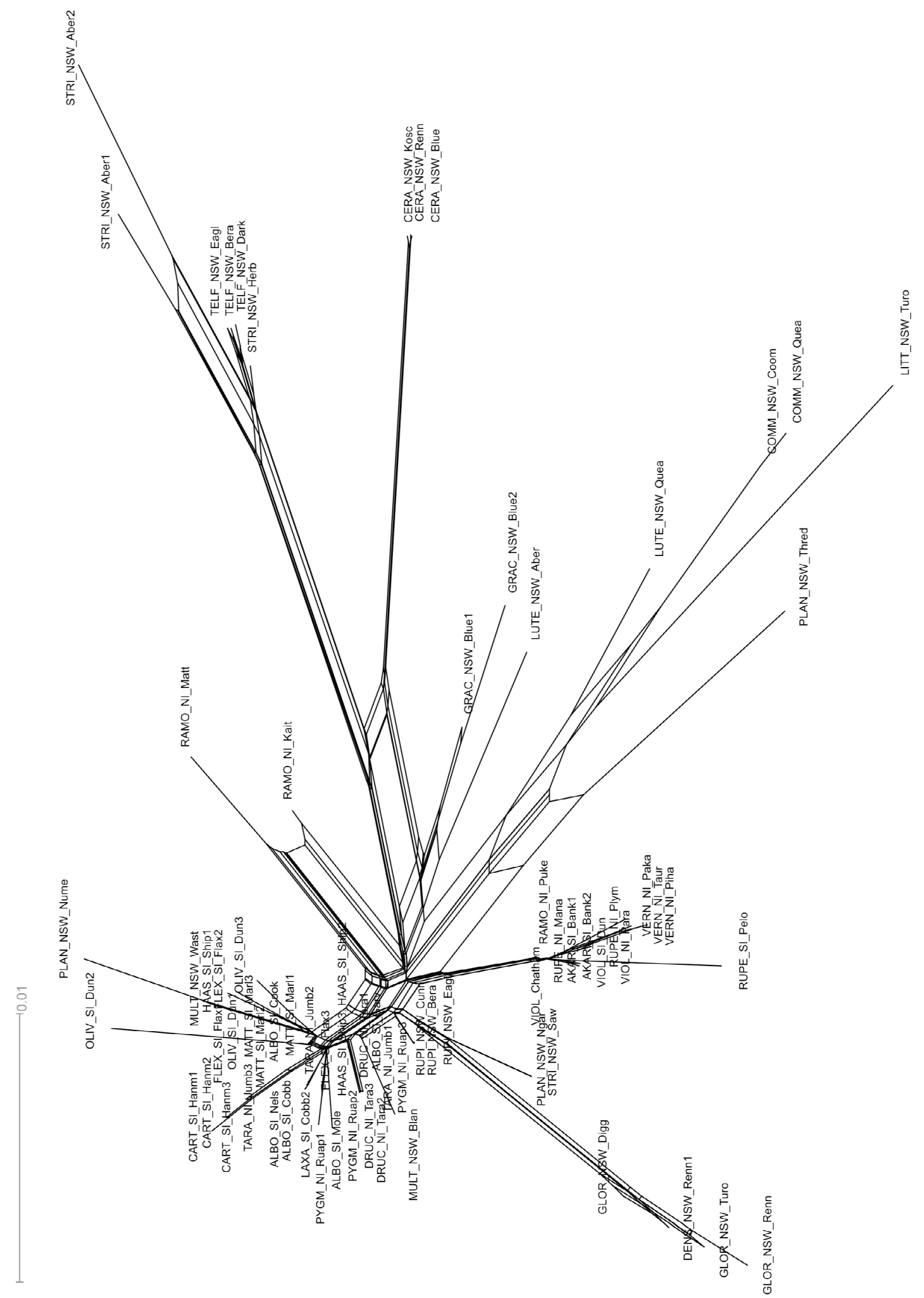


Appendix 2.2 Neighbour net analysis of the $\operatorname{trn} L-F+\operatorname{trn} K$ dataset from chapter three created using SplitsTree v 4.10

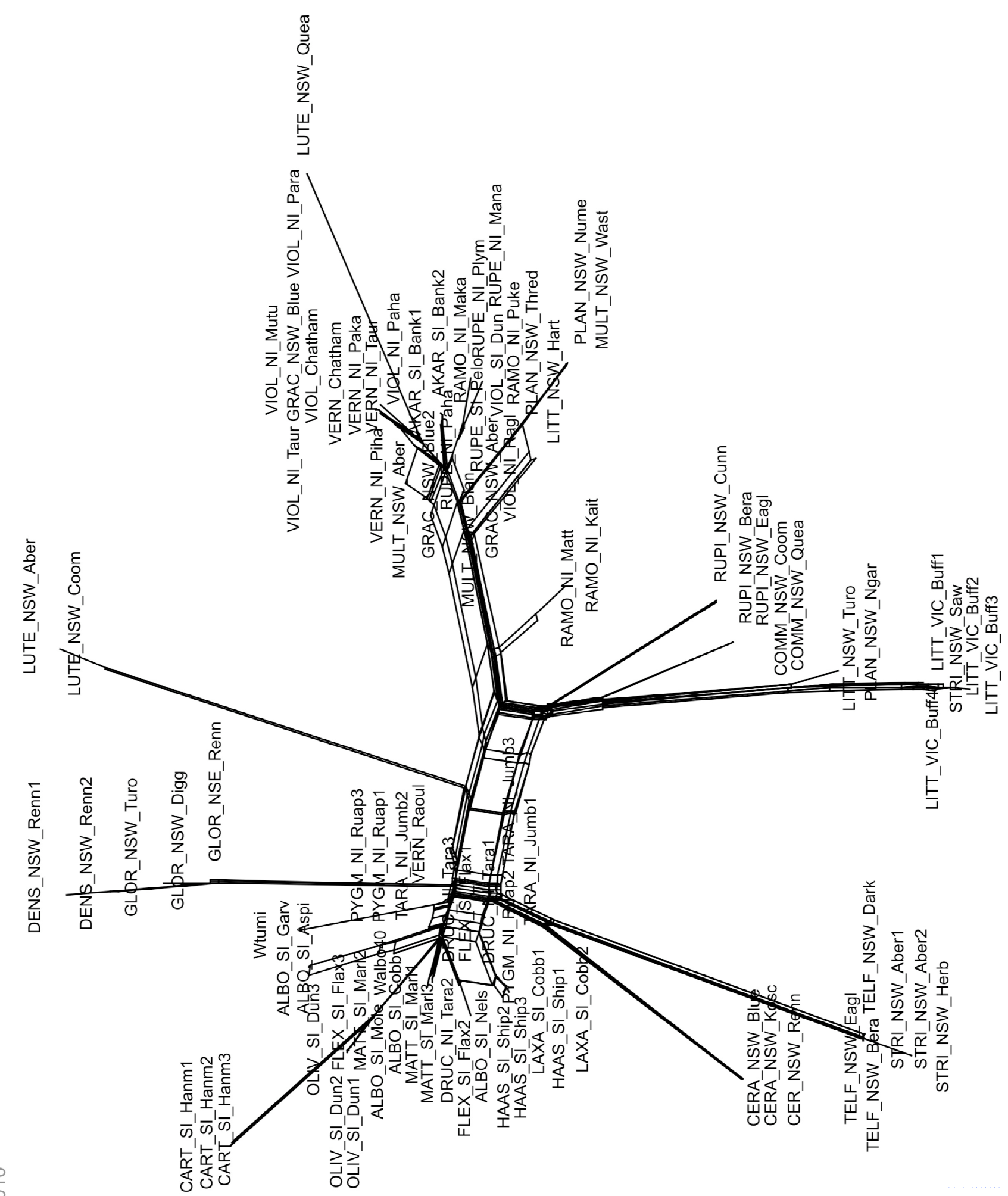


Appensix 2.3 Neighbour net analysis of the combined ITS $+\operatorname{trn} L-F+\operatorname{trn} K$ dataset from chapter three created using SplitsTree v 4.10

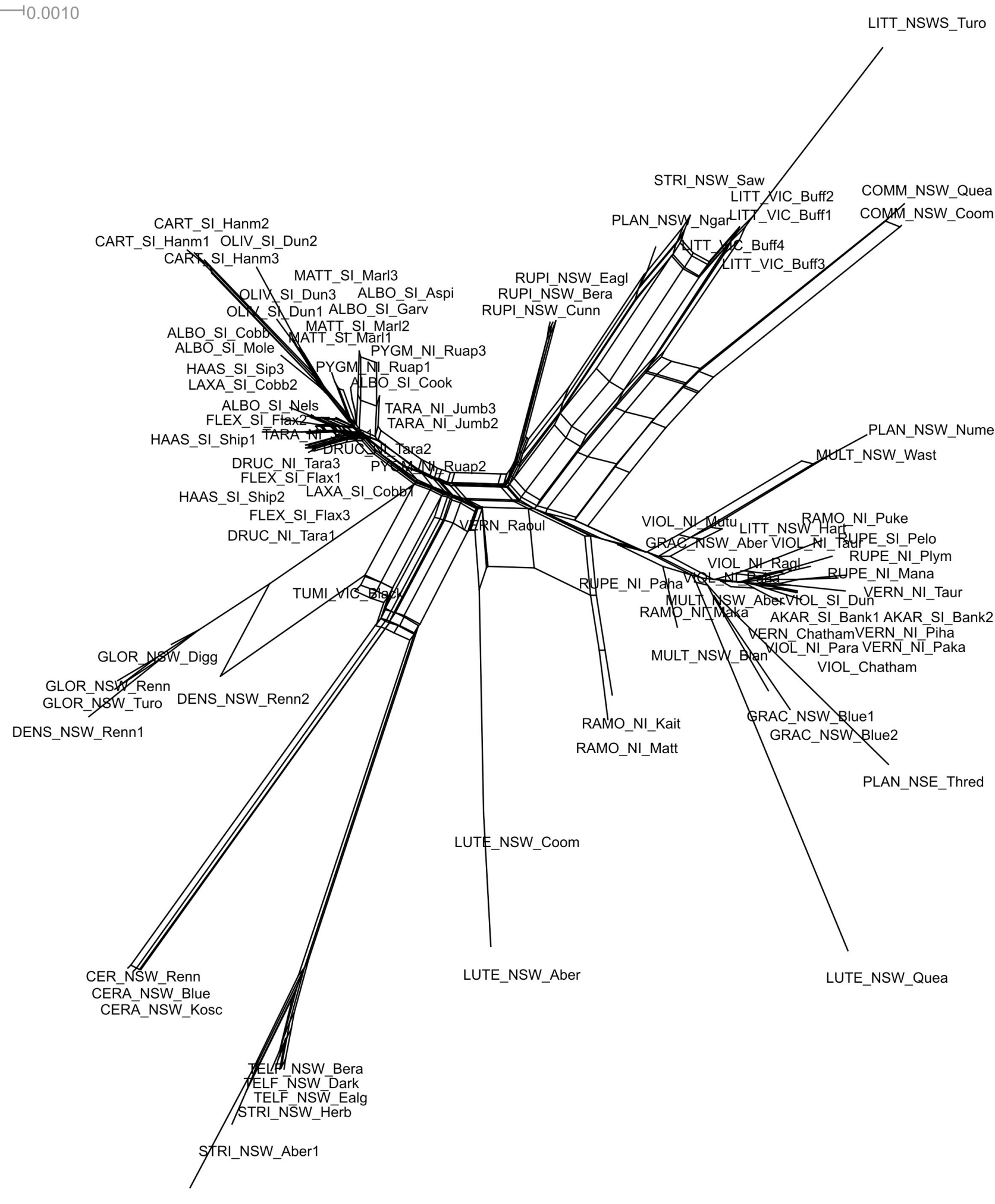

STRI_NSW_Aber2 